\title{
Two-sided bounds for degenerate processes with densities supported in subsets of $\mathbb{R}^{N}$
}

\author{
Chiara Cinti* Stéphane Menozzi ${ }^{\dagger}$ And Sergio Polidoro ${ }^{\ddagger}$
}

Abstract: We obtain two-sided bounds for the density of stochastic processes satisfying a weak Hörmander condition. In particular we consider the cases when the support of the density is not the whole space and when the density has various asymptotic regimes depending on the starting/final points considered (which are as well related to the number of brackets needed to span the space in Hörmander's theorem). The proofs of our lower bounds are based on Harnack inequalities for positive solutions of PDEs whereas the upper bounds are derived from the probabilistic representation of the density given by the Malliavin calculus.

Keywords: Harnack inequality, Malliavin Calculus, Hörmander condition, two-sided bounds. 2000 Mathematics Subject Classification: Primary 35H10, 60J60, secondary 31C05, 60H07.

\section{Introduction}

We present a methodology to derive two-sided bounds for the density of some $\mathbb{R}^{N}$-valued degenerate processes of the form

$$
X_{t}=x+\sum_{i=1}^{n} \int_{0}^{t} Y_{i}\left(X_{s}\right) \circ d W_{s}^{i}+\int_{0}^{t} Y_{0}\left(X_{s}\right) d s
$$

where the $\left(Y_{i}\right)_{i \in \llbracket 0, n \rrbracket}$ are smooth vector fields defined on $\mathbb{R}^{N},\left(\left(W_{t}^{i}\right)_{t \geq 0}\right)_{i \in \llbracket 1, n \rrbracket}$ stand for $n$ standard monodimensional independent Brownian motions defined on a filtered probability space $\left(\Omega, \mathscr{F},\left(\mathscr{F}_{t}\right)_{t \geq 0}, \mathbb{P}\right)$ satisfying the usual conditions. Also $\circ d W_{t}$ denotes the Stratonovitch integral. The above stochastic differential equation is associated to the Kolmogorov operator

$$
\mathscr{L}=\frac{1}{2} \sum_{i=1}^{n} Y_{i}^{2}+Z, \quad Z=Y_{0}-\partial_{t} .
$$

We assume that the Hörmander condition holds:

*Dipartimento di Matematica, Università di Bologna, Piazza di Porta S. Donato 5, 40126 Bologna (Italy). E-mail: cinti@dm.unibo.it

${ }^{\dagger}$ Université d'Evry Val d'Essonne, Laboratoire d'Analyse et Probabilits, 23 Boulevard de France, 91037 Evry Cedex (France). E-mail: stephane.menozzi@univ-evry.fr

${ }^{\ddagger}$ Dipartimento di Scienze Fisiche, Informatiche e Matematiche, Università di Modena e Reggio Emilia, via Campi 213/b, 41125 Modena (Italy). E-mail: sergio.polidoro@unimore.it 


$$
\operatorname{Rank}\left(\operatorname{Lie}\left\{Y_{1}, \cdots, Y_{n}, Z\right\}(x)\right)=N+1, \quad \forall x \in \mathbb{R}^{N} .
$$

We will particularly focus on processes satisfying a weak Hörmander condition, that is $\operatorname{Rank}\left(\operatorname{Lie}\left\{Y_{1}, \cdots, Y_{n},-\partial_{t}\right\}(x)\right)<N+1, \forall x \in \mathbb{R}^{N}$. This means that the first order vector field $Y_{0}$ (or equivalently the drift term of the SDE) is needed to span all the directions.

As leading examples we have in mind processes of the form

$$
X_{t}^{i}=x_{i}+W_{t}^{i}, \quad \forall i \in \llbracket 1, n \rrbracket, \quad X_{t}^{n+1}=x_{n+1}+\int_{0}^{t}\left|X_{s}^{1, n}\right|^{k} d s
$$

where $X_{s}^{1, n}=\left(X_{s}^{1}, \cdots, X_{s}^{n}\right)$ (and correspondingly for every $x \in \mathbb{R}^{n+1}, x_{1, n}:=\left(x_{1}, \cdots, x_{n}\right)$ ), $k$ is any even positive integer and |.| denotes the Euclidean norm of $\mathbb{R}^{n}$. Note that we only consider even exponents in (1.3) in order to keep $Y_{0}$ smooth. Our approach also applies to

$$
X_{t}^{i}=x_{i}+W_{t}^{i}, \quad \forall i \in \llbracket 1, n \rrbracket, \quad X_{t}^{n+1}=x_{n+1}+\int_{0}^{t} \sum_{i=1}^{n}\left(X_{s}^{i}\right)^{k} d s,
$$

for any given positive integer $k$.

It is easily seen that the above class of processes satisfies the weak Hörmander condition. Also for equation (1.3), the density $p(t, x,$.$) of X_{t}$ is supported on $\mathbb{R}^{n} \times\left(x_{n+1},+\infty\right)$ for any $t>0$. Analogously, for equation (1.4), the support of $p(t, x,$.$) is \mathbb{R}^{n+1}$ when $k$ is odd and $\mathbb{R}^{n} \times\left(x_{n+1},+\infty\right)$ when $k$ is even.

Let us now briefly recall some known results concerning these two examples. First of all, for $k=1$, equation (1.4) defines a Gaussian process. The explicit expression of the density goes back to Kolmogorov [25] and writes for all $t>0, x, \xi \in \mathbb{R}^{n+1}$ :

$$
p_{K}(t, x, \xi)=\frac{\sqrt{3}}{(2 \pi)^{\frac{n+1}{2}} t^{\frac{n+3}{2}}} \exp \left(-\left\{\frac{1}{4} \frac{\left|\xi_{1, n}-x_{1, n}\right|^{2}}{t}+3 \frac{\left|\xi_{n+1}-x_{n+1}-\frac{\sum_{i=1}^{n}\left(x_{i}+\xi_{i}\right)}{2} t\right|^{2}}{t^{3}}\right\}\right) .
$$

We already observe the two time scales associated respectively to the Brownian motion (of order $t^{1 / 2}$ ) and to its integral (of order $t^{3 / 2}$ ) which give the global diagonal decay of order $t^{n / 2+3 / 2}$. The additional term $\frac{x_{1}+\xi_{1}}{2} t$ in the above estimate is due to the transport of the initial condition by the unbounded drift. We also refer to the works of Cinti and Polidoro [17] and Delarue and Menozzi [19] for similar estimates in the more general framework of variable coefficients, including non linear drift terms with linear growth.

For equation (1.3) and $k=2, n=1$, a representation of the density of $X_{t}$ has been obtained from the seminal works of Kac on the Laplace transform of the integral of the square of the Brownian motion [23]. We can refer to the monograph of Borodin and Salminen [10] for an explicit expression in terms of special functions. We can also mention the work of Tolmatz [39] concerning the distribution function of the square of the Brownian bridge already characterized in the early work of Smirnov [36]. Anyhow, all these explicit representations are very much linked to Liouville type problems and this approach can hardly be extended to higher dimensions for the underlying Brownian motion. Also, it seems difficult from the expressions of [10] to derive explicit quantitative bounds on the density. 
Some related examples have been addressed by Ben Arous and Léandre [5] who obtained asymptotic expansions for the density on the diagonal for the process $X_{t}^{1}=x_{1}+W_{t}^{1}, X_{t}^{2}=$ $x_{2}+\int_{0}^{t}\left(X_{s}^{1}\right)^{m} d W_{s}^{2}+\int_{0}^{t}\left(X_{s}^{1}\right)^{k} d s$. Various asymptotic regimes are deduced depending on $m$ and $k$. Anyhow, the strong Hörmander condition is really required in their approach, i.e. the stochastic integral is needed in $X^{2}$.

From the applicative point of view, equations with quadratic growth naturally appear in some turbulence models, see e.g. the chapter concerning the dyadic model in Flandoli [20]. This model is derived from the formulation of the Euler equations on the torus in Fourier series after a simplification consisting in considering a nearest neighbour interaction in the wave space. This operation leads to consider an infinite system of differential equations whose coefficients have quadratic growth. In order to obtain some uniqueness properties, a Brownian noise is usually added on each component. In the current work, we investigate from a quantitative viewpoint what can be said for a drastic reduction of this simplified model, that is when considering 2 equations only, when the noise only acts on one component and is transmitted through the system thanks to the (weak) Hörmander condition.

Our approach to derive two-sided estimates for the above examples is the following. The lower bounds are obtained using local Harnack estimates for positive solutions of $\mathscr{L} u=0$ with $\mathscr{L}$ defined in (1.2). Once the Harnack inequality is established, the lower bound for $p(t, x, \xi)$ is derived applying it recursively along a suitable path joining $x$ to $\xi$ in time $t$. The set of points of the path to which the Harnack inequality is applied is commonly called a Harnack chain. For $k=1$ in (1.4) the path can be chosen as the solution to the deterministic controllability problem associated to (1.4), that is taking the points of the Harnack chain along the path $\gamma$ where

$$
\gamma_{i}^{\prime}(s)=\omega_{i}(s), \forall i \in \llbracket 1, n \rrbracket, \gamma_{n+1}^{\prime}(s)=\sum_{i=1}^{n} \gamma_{i}(s), \gamma(0)=x, \gamma(t)=\xi .
$$

and $\omega: L^{2}([0, t]) \rightarrow \mathbb{R}^{n}$ achieves the minimum of $\int_{0}^{t}|\omega(s)|^{2} d s$, see e.g. Boscain and Polidoro [11], Carciola et al. [13] and Delarue and Menozzi [19].

In the more general case $k>1$ it is known that uniqueness fails for the associated control problem, i.e. when $\gamma_{n+1}^{\prime}(s)=\sum_{i=1}^{n}\left(\gamma_{i}(s)\right)^{k}$ in the above equation (see e.g. Trélat [40]). Therefore, there is not a single natural choice for the path $\gamma$. Actually, we will consider suitable paths in order to derive homogeneous two-sided bounds. After the statement of our main results, we will see in Remark 2.2 that the paths we consider allow to obtain a cost similar to the one found in [40] for the abnormal extremals of the value function associated to the control problem.

Anyhow, the crucial point in this approach is to obtain a Harnack inequality invariant w.r.t. scale and translation. Introducing for all $(m, x) \in \mathbb{N}^{*} \times \mathbb{R}^{n+1}$ the space $V_{m}(x):=$ $\left\{\left(\left(Y_{i_{1}}\right)_{i_{1} \in \llbracket 1, n \rrbracket},\left(\left[Y_{i_{1}}, Y_{i_{2}}\right](x)\right)_{\left(i_{1}, i_{2}\right) \in \llbracket 0, n \rrbracket^{2}}, \cdots,\left(\left[Y_{i_{1}},\left[Y_{i_{2}}, \cdots,\left[Y_{i_{m-1}}, Y_{i_{m}}\right]\right]\right](x)\right)_{\left(i_{1}, \cdots, i_{m}\right) \in \llbracket 0, n \rrbracket m}\right)\right\}$, the above invariance properties imply that $\operatorname{dim}\left(\operatorname{Span}\left\{V_{m}(x)\right\}\right)$ does not depend on $x$ for any $m$. This property fails for $k>1$ since we need exactly $k$ brackets to span the space at $x=\left(0_{1, n}, x_{n+1}\right)$ and exactly one bracket elsewhere. Hence, we need to consider a lifting procedure of $\mathscr{L}$ in (1.2) introduced by Rotschild and Stein [35] (see also Bonfiglioli and Lanconelli [6]). Our strategy then consists in obtaining an invariant Harnack inequality 
for the lifted operator $\widetilde{\mathscr{L}}$. We then conclude applying the previous Harnack inequality to $\mathscr{L}$-harmonic functions (which are also $\widetilde{\mathscr{L}}$-harmonic). A first attempt to achieve the whole procedure to derive a lower bound for (1.4) and odd $k$ can be found in Cinti and Polidoro $[16]$.

Concerning the upper bounds, we rely on the representation of the density of $p$ obtained by the Malliavin calculus. We refer to Nualart [33] for a comprehensive treatment of this subject. The main issues then consist in controlling the tails of the random variables at hand and the $L^{p}$ norm of the Malliavin covariance matrix for $p \geq 1$. The tails can be controlled thanks to some fine properties of the Brownian motion or bridge and its local time. The behavior of the Malliavin covariance matrix has to be carefully analyzed introducing a dichotomy between the case for which the final and starting points of the Brownian motion in (1.3)-(1.4) are close to zero w.r.t. the characteristic time-scale, i.e. $\left|x_{1, n}\right| \vee\left|\xi_{1, n}\right| \leq K t^{1 / 2}$ for a given $K>0$, which means that the non-degenerate component is in diagonal regime, and the complementary set. In the first case, we will see that the characteristic time scales of the system (1.3), (1.4) and the probabilistic approach to the proof of Hörmander theorem, see e.g. Norris [31] will lead to the expected bound on the Malliavin covariance matrix whereas in the second case a more subtle analysis is required in order to derive a diagonal behavior of the density similar to the Gaussian case (1.5). Intuitively, when the magnitude of either the starting or the final point of the Brownian motion is above the characteristic time-scale, then only one bracket is needed to span the space and the Gaussian regime prevails in small time.

Note that our procedure can be split in two steps. In the first one, purely PDEs methods provide us with lower bounds of the density $p$. In this part useful information about its asymptotic behavior in various regimes are obtained by elementary arguments. Once the lower bounds have been established, we rely on some ad hoc tools of the Malliavin calculus to prove the analogous upper bounds. However, aiming at improving the readability of our work, we reverse our exposition: we first prove the upper bounds, as well as the diagonal ones, by using probabilistic methods, then we prove the lower bounds by PDEs arguments.

The article is organized as follows. We state our main results in Section 2. We then recall some basic facts of Malliavin calculus in Section 3 and obtain the upper bounds as well as a diagonal lower bound in Gaussian regime in Section 4.In Section 5, we recall some aspects of abstract potential theory needed to derive the invariant Harnack inequality. We also give a geometric characterization of the set where the inequality holds. Section 6 is devoted to the proof of the lower bounds.

\section{Main Results}

Before giving the precise statement of our bounds for the the density $p$ of $X$ in (1.3) or (1.4), we give some remarks. In the sequel $p(t, x,$.$) stands for the density of any stochastic process$ $X$ at time $t$ starting from $x$. It is well known that, if the vector fields $Y_{1}, \ldots, Y_{n}$ (note that the drift term $Y_{0}$ does not appear) satisfy the Hörmander condition, then the following two sided bound holds:

$$
p(t, x, \xi) \asymp \frac{1}{\sqrt{\operatorname{vol}\left(B_{Y}(x, t)\right)}} \exp \left(-\frac{d_{Y}(x, \xi)^{2}}{t}\right) .
$$


Here and in the sequel, for measurable functions $g: \mathbb{R}^{+*} \times \mathbb{R}^{n} \rightarrow \mathbb{R}, h: \mathbb{R}^{+*} \times \mathbb{R}^{2 n}$, the above notation $p(t, x, \xi) \asymp \frac{1}{g(t, x)} \exp (-h(t, x, \xi))$ means that there exists a constant $C \geq 1$ s.t.

$$
\frac{C^{-1}}{g(t, x)} \exp (-C h(t, x, \xi)) \leq p(t, x, \xi) \leq \frac{C}{g(t, x)} \exp \left(-C^{-1} h(t, x, \xi)\right) .
$$

Moreover, in (2.1), $d_{Y}$ denotes the Carnot-Carathéodory distance associated to $Y_{1}, \ldots, Y_{n}$, and $B_{Y}(x, r)$ is the relevant metric ball, with center at $x$ and radius $r$. On the other hand (1.5) shows that, when the drift term $Y_{0}$ is needed to check the Hörmander condition, the density $p$ of the process $X$ doesn't satisfy (2.1). In this article we prove that, when considering processes (1.3) and (1.4) with $k>1$, different asymptotic behavior as $|x| \rightarrow+\infty$ appear.

To be more specific, we first remark that a behavior similar to (1.5) can also be observed for equations (1.3) and (1.4).Conditioning w.r.t. to the non degenerate component we get

$$
p(t, x, \xi)=p_{X^{1, n}}\left(t, x_{1, n}, \xi_{1, n}\right) p_{X^{n+1}}\left(t, x_{n+1}, \xi_{n+1} \mid X_{0}^{1, n}=x_{1, n}, X_{t}^{1, n}=\xi_{1, n}\right),
$$

where $p_{X^{1, n}}\left(t, x_{1, n}, \xi_{1, n}\right)=\frac{1}{(2 \pi t)^{n / 2}} \exp \left(-\frac{\left|\xi_{1, n}-x_{1, n}\right|^{2}}{2 t}\right)$ is the usual Gaussian density,

$$
\begin{gathered}
p_{X^{n+1}}\left(t, x_{n+1}, \xi_{n+1} \mid X_{0}^{1, n}=x_{1, n}, X_{t}^{1, n}=\xi_{1, n}\right)=p_{Y_{t}}\left(\xi_{n+1}-x_{n+1}\right), \\
Y_{t}:=\left\{\begin{array}{l}
\int_{0}^{t}\left|\frac{t-s}{t} x_{1, n}+\frac{s}{t} \xi_{1, n}+W_{s}^{0, t}\right|^{k} d s \text { for }(1.3), \\
\int_{0}^{t} \sum_{i=1}^{n}\left(\frac{t-s}{t} x_{i}+\frac{s}{t} \xi_{i}+W_{s}^{0, t, i}\right)^{k} d s \text { for }(1.4),
\end{array}\right.
\end{gathered}
$$

and $\left(W_{s}^{0, t}\right)_{u \in[0, t]}$ stands for the standard $d$-dimensional Brownian bridge on $[0, t]$, i.e. starting and ending at 0 .

For the sake of simplicity, we next focus on the case $n=1$ and $k=2$ so that (1.3) and (1.4) coincide. Moreover we assume $x_{1}=\xi_{1}$. This leads to estimate the density of:

$$
Y_{t}:=\int_{0}^{t}\left(x_{1}+W_{s}^{0, t}\right)^{2} d s=t x_{1}^{2}+2 x_{1} \int_{0}^{t} W_{s}^{0, t} d s+\int_{0}^{t}\left(W_{s}^{0, t}\right)^{2} d s .
$$

Thus, when $\left|x_{1}\right|$ is sufficiently big w.r.t. the characteristic time scale $t^{1 / 2}$, the Gaussian random variable

$$
G:=2 x_{1} \int_{0}^{t} W_{s}^{0, t} d s \stackrel{(\text { law })}{=} \mathcal{N}\left(0, \frac{t^{3}}{3}\left|x_{1}\right|^{2}\right)
$$

dominates in terms of fluctuation order w.r.t. the other random contribution whose variance behaves as $O\left(t^{4}\right)^{1}$.

If we additionally assume that $\left|\xi_{2}-x_{2}-t x_{1}^{2}\right| \leq \bar{C}\left|x_{1}\right| t^{3 / 2}$, for some constant $\bar{C}:=\bar{C}(n=$ $1, k=2)$ to be specified later on, that is the deviation from the deterministic system deriving

\footnotetext{
${ }^{1}$ The previous identity in law is derived from Itô's formula and the differential dynamics of the Brownian bridge. Namely, $\int_{0}^{t} W_{s}^{0, t} d s=\left\{-\left.(t-s) W_{s}^{0, t}\right|_{0} ^{t}+\int_{0}^{t}(t-s)\left(-\frac{W_{s}^{0, t}}{t-s} d s+d W_{s}\right)\right\} \Longleftrightarrow \int_{0}^{t} W_{s}^{0, t} d s=\frac{1}{2} \int_{0}^{t}(t-$ $s) d W_{s}$.
} 
from (1.3), obtained dropping the Brownian contribution, has the same order as the standard deviation of $G$, we actually find:

$$
p(t, x, \xi) \asymp \frac{1}{\left|x_{1}\right| t^{\frac{1+3}{2}}} \exp \left(-\left\{\frac{\left|\xi_{1}-x_{1}\right|^{2}}{t}+\frac{\left|\xi_{2}-x_{2}-x_{1}^{2} t\right|^{2}}{\left|x_{1}\right|^{2} t^{3}}\right\}\right) .
$$

When $\left|\xi_{2}-x_{2}-t x_{1}^{2}\right|>\bar{C}\left|x_{1}\right| t^{3 / 2}$, that is when the deviation from the deterministic system exceeds a certain constant times the standard deviation, the term $\int_{0}^{t}\left(W_{s}^{0, t}\right)^{2} d s$ in (2.3) is not negligeable any more and we obtain the following heavy-tailed estimate:

$$
p(t, x, \xi) \asymp \frac{1}{t^{\frac{1}{2}+2}} \exp \left(-\left\{\frac{\left|\xi_{1}-x_{1}\right|^{2}}{t}+\frac{\left|\xi_{2}-x_{2}-x_{1}^{2} t\right|}{t^{2}}\right\}\right) .
$$

The diagonal contribution of the degenerate component corresponds to the intrinsic scale of order $t^{2}$ of the term $\int_{0}^{t}\left(W_{s}^{0, t}\right)^{2} d s$. In particular, if $x_{1, n}=0_{1, n}$ this is the only random variable involved. The off-diagonal bound can be explained by the fact that $\int_{0}^{t}\left(W_{s}^{0, t}\right)^{2} d s$ belongs to the Wiener chaos of order 2. The tails of the distribution function for such random variables can be characterized, see e.g. Janson [22], and are homogeneous to the non Gaussian term in the above estimate.

Observe also that the density $p$ is supported on the half space $\left\{\xi \in \mathbb{R}^{2}: \xi_{2}>x_{2}\right\}$. We obtain as well an asymptotic behavior for the density close to the boundary. Precisely, for $0<\xi_{2}-x_{2}$ sufficiently small w.r.t. to the characteristic time-scale $t^{2}$ of $\int_{0}^{t}\left(W_{s}^{0, t}\right)^{2} d s$, that is when the deviations of the degenerate component have the same magnitude as those of the highest order random contribution, then

$$
p(t, x, \xi) \asymp \frac{1}{t^{1 / 2+2}} \exp \left(-\left\{\frac{\left|x_{1}\right|^{4}+\left|\xi_{1}\right|^{4}}{\xi_{2}-x_{2}}+\frac{t^{2}}{\left(\xi_{2}-x_{2}\right)}\right\}\right) .
$$

We summarize the above remarks with the assertion that processes of the form (1.3) or (1.4) do not have a single regime for $k>1$. The precise statements of the previous density bounds are formulated for general $n$ and $k$ in the following Theorem 2.1.

Theorem 2.1 Let $x=\left(x_{1, n}, x_{n+1}\right) \in \mathbb{R}^{n+1}$, and $\xi=\left(\xi_{1, n}, \xi_{n+1}\right) \in \mathbb{R}^{n} \times\left(x_{n+1},+\infty\right)$ for $k$ even, and $\xi \in \mathbb{R}^{n+1}$ for $k$ odd, be given. Define

$$
\Psi\left(x_{1, n}, \xi_{1, n}\right):=\left\{\begin{array}{l}
\left|x_{1, n}\right|^{k}+\left|\xi_{1, n}\right|^{k}, \text { for }(1.3), \\
\sum_{i=1}^{n}\left\{\left(x_{i}\right)^{k}+\left(\xi_{i}\right)^{k}\right\}, \text { for (1.4). }
\end{array}\right.
$$

i) Assume $\frac{\left|\xi_{n+1}-x_{n+1}-c t\left(\left|x_{1, n}\right|^{k}+\left|\xi_{1, n}\right|^{k}\right)\right|}{t^{3 / 2}\left(\left|x_{1, n}\right|^{k-1}+\left|\xi_{1, n}\right|^{k-1}\right)} \geq \bar{C}$ where $c:=c(k)=2+\frac{2^{k-1}}{k+1}$ and $\bar{C}$ is fixed. Then there exists a constant $C_{1}:=C_{1}(n, k, \bar{C}) \geq 1$ s.t. for every $t>0$,

$$
\begin{array}{r}
\frac{C_{1}^{-1}}{t^{\frac{n+k}{2}}+1} \exp \left(-C_{1} I\left(t, x, \xi, \frac{1}{2^{k+4}}\right)\right) \leq p(t, x, \xi) \leq \frac{C_{1}}{t^{\frac{n+k}{2}}+1} \exp \left(-C_{1}^{-1} I\left(t, x, \xi, \frac{2^{k-1}}{k+1}\right)\right),(2.4) \\
\forall c \in \mathbb{R}^{+}, I(t, x, \xi, c) \quad:=\frac{\left|\xi_{1, n}-x_{1, n}\right|^{2}}{t}+\frac{\left|\xi_{n+1}-x_{n+1}-c \Psi\left(x_{1, n}, \xi_{1, n}\right) t\right|^{2 / k}}{t^{1+2 / k}} .
\end{array}
$$


ii) Assume $\frac{\left|\xi_{n+1}-x_{n+1}-c t\left(\left|x_{1, n}\right|^{k}+\left|\xi_{1, n}\right|^{k}\right)\right|}{t^{3 / 2}\left(\left|x_{1, n}\right|^{k-1}+\left|\xi_{1, n}\right|^{k-1}\right)} \leq \bar{C}$ (with $c, \bar{C}$ as in point i)) and $\left|x_{1, n}\right| \vee\left|\xi_{1, n}\right| / t^{1 / 2} \geq$ $K$, with $K$ sufficiently large. Then, there exists $C_{2}:=C_{2}(n, k, K, \bar{C}) \geq 1$ s.t. for every $t>0$ :

$$
\begin{aligned}
\frac{C_{2}^{-1} \exp \left(-C_{2} I(t, x, \xi)\right)}{\left(\left|x_{1, n}\right|^{k-1}+\left|\xi_{1, n}\right|^{k-1}\right) t^{\frac{n+3}{2}}} & \leq p(t, x, \xi) \leq \frac{C_{2} \exp \left(-C_{2}^{-1} I(t, x, \xi)\right)}{\left(\left.\left|x_{1, n}\right|\right|^{k-1}+\left|\xi_{1, n}\right|^{k-1}\right) t^{\frac{n+3}{2}}} \\
I(t, x, \xi) & :=\frac{\left|\xi_{1, n}-x_{1, n}\right|^{2}}{t}+\frac{\left|\xi_{n+1}-x_{n+1}-\Psi\left(x_{1, n}, \xi_{1, n}\right) t\right|^{2}}{\left(\left|x_{1, n}\right|^{(k-1)}+\left|\xi_{1, n}\right|^{(k-1)}\right)^{2} t^{3}} .
\end{aligned}
$$

iii) For $t>0$, assume $\left|\xi_{n+1}-x_{n+1}\right| \leq K t^{1+k / 2}$ for sufficiently small $K$. Then, there exists $C_{3}:=C_{3}(n, k, K) \geq 1$ s.t. we have:

$$
\begin{aligned}
\frac{C_{3}^{-1}}{t^{\frac{n+k}{2}}+1} \exp \left(-C_{3} I(t, x, \xi)\right) & \leq p(t, x, \xi) \leq \frac{C_{3}}{t^{\frac{n+k}{2}}+1} \exp \left(-C_{3}^{-1} I(t, x, \xi)\right), \\
I(t, x, \xi) & :=\frac{\left|x_{1, n}\right|^{2+k}+\left|\xi_{1, n}\right|^{2+k}}{\left|\xi_{n+1}-x_{n+1}\right|}+\frac{t^{1+2 / k}}{\left|\xi_{n+1}-x_{n+1}\right|^{2 / k}} .
\end{aligned}
$$

As already pointed out, processes of the form (1.3) or (1.4) do not have a single regime anymore for $k>1$.

Let us anyhow specify that when $C^{-1} \sqrt{t} \leq\left|x_{i}\right| \leq C \sqrt{t}, \forall i \in \llbracket 1, n \rrbracket, C \geq 1$, then expanding $Y_{t}$ as in (2.3), we find that all the terms have the same order and thus a global estimate of type (2.4) (resp. of type (2.5)) holds for the upper bound (resp. lower bound) in both cases (1.3) and (1.4). Observe also that in this case (2.4) and (2.5) give the same global diagonal decay of order $t^{(k+n) / 2+1}$.

Remark 2.2 As already mentioned in the introduction, for $k=2, n=1$, we observe from (2.6) that the off-diagonal bound is homogeneous to the asymptotic expansion of the value function associated to the control problem at its abnormal extremals, see Example 4.2 in [40]. The optimal cost is asymptotically equivalent to $\frac{1}{4} \frac{\xi_{1}^{4}}{\xi_{2}}$ when $x=(0,0)$ as $\xi$ is close to $(0,0)$.

Remark 2.3 Fix $\left|\xi_{n+1}-x_{n+1}\right|$ small, $t \in\left[K^{-1}\left|\xi_{n+1}-x_{n+1}\right|^{2-\varepsilon}, K\left|\xi_{n+1}-x_{n+1}\right|^{2-\varepsilon}\right]$ for given $K \geq 1, \varepsilon \geq 0$. We then get from (2.6) that there exist $\widetilde{c}:=\widetilde{c}(n, k), \widetilde{C}:=\widetilde{C}(n, k, T)$ s.t. $p(t, x, \xi) \leq \widetilde{C} \exp \left(-\widetilde{c} /\left|\xi_{n+1}-x_{n+1}\right|^{\varepsilon}\right)$. This estimate can be compared to the exponential decay on the diagonal proved by Ben Arous and Léandre in [5, Theorem 1.1].

\section{A Glimpse of Malliavin Calculus}

\subsection{Introduction}

Introduced at the end of the 70s by Malliavin, [30], [29], the stochastic calculus of variations, now known as Malliavin calculus, turned out to be a very fruitful tool. It allows to give probabilistic proofs of the celebrated Hörmander theorem, see e.g. Stroock [37] or Norris 
[31]. It also provides a quite natural way to derive density estimates for degenerate diffusion processes. The most striking achievement in this direction is the series of papers by Kusuoka and Stroock, [26], [27], [28]. Anyhow, in those works the authors always considered "strong" Hörmander conditions, that is the underlying space is assumed to be spanned by brackets involving only the vector fields of the diffusive part. For the examples (1.3), (1.4) we consider, this condition is not fulfilled. Anyhow a careful analysis of the Malliavin covariance matrix will naturally lead to the upper bounds of Theorem 2.1 and also to a Gaussian lower bound, when the initial or final point of the non-degenerate component is "far" from zero w.r.t. the characteristic time scale on the compact sets of the underlying metric, see point ii) of Theorem 2.1.

We also point out that because of the non uniqueness associated to the deterministic control problem, the strategy of [19] relying on a stochastic control representation of the density breaks down. For the systems handled in [19], we refer to Bally and Kohatsu-Higa for a Malliavin calculus approach [2]. The Malliavin calculus remains the most robust probabilistic approach to density estimate in the degenerate setting.

We now briefly state some facts and notations concerning the Malliavin calculus that are needed to prove our results. We refer to the monograph of Nualart [32], from which we borrow the notations, or Chapter 5 in Ikeda and Watanabe [21], for further details.

\subsection{Operators of the Malliavin Calculus}

Let us consider an $n$-dimensional Brownian motion $W$ on the filtered probability space $\left(\Omega, \mathscr{F},\left(\mathscr{F}_{t}\right)_{t \geq 0}, \mathbb{P}\right)$ and a given $T>0$. Define for $h \in L^{2}\left(\mathbb{R}^{+}, \mathbb{R}^{n}\right), W(h)=\int_{0}^{T}\left\langle h(s), d W_{s}\right\rangle$. We denote by $\mathcal{S}$ the space of simple functionals of the Brownian motion $W$, that is the subspace of $L^{2}(\Omega, \mathscr{F}, \mathbb{P})$ consisting of real valued random variables $F$ having the form

$$
F=f\left(W\left(h_{1}\right), \cdots, W\left(h_{m}\right)\right),
$$

for some $m \in \mathbb{N}, h_{i} \in L^{2}\left(\mathbb{R}^{+}, \mathbb{R}^{n}\right)$, and where $f: \mathbb{R}^{m} \rightarrow \mathbb{R}$ stands for a smooth function with polynomial growth.

\section{Malliavin Derivative.}

For $F \in \mathcal{S}$, we define the Malliavin derivative $\left(D_{t} F\right)_{t \in[0, T]}$ as the $\mathbb{R}^{n}$-dimensional (non adapted) process

$$
D_{t} F=\sum_{i=1}^{m} \partial_{x_{i}} f\left(W\left(h_{1}\right), \cdots, W\left(h_{m}\right)\right) h_{i}(t) .
$$

For any $q \geq 1$, the operator $D: \mathcal{S} \rightarrow L^{q}\left(\Omega, L^{2}(0, T)\right)$ is closable. We denote its domain by $\mathbb{D}^{1, q}$ which is actually the completion of $\mathcal{S}$ w.r.t. the norm

$$
\|F\|_{1, q}:=\left\{\mathbb{E}\left[|F|^{q}\right]+\mathbb{E}\left[|D F|_{L^{2}(0, T)}^{q}\right]\right\}^{1 / q} .
$$

Writing $D_{t}^{j} F$ for the $j^{\text {th }}$ component of $D_{t} F$, we define the $k^{\text {th }}$ order derivative as the random vector on $[0, T]^{k} \times \Omega$ with coordinates:

$$
D_{t_{1}, \cdots, t_{k}}^{j_{1}, \cdots, j_{k}} F:=D_{t_{k}}^{j_{k}} \cdots D_{t_{1}}^{j_{1}} F
$$


We then denote by $\mathbb{D}^{N, q}$ the completion of $\mathcal{S}$ w.r.t. the norm

$$
\|F\|_{N, q}:=\left\{\mathbb{E}\left[|F|^{q}\right]+\sum_{k=1}^{N} \mathbb{E}\left[\left|D^{k} F\right|_{L^{2}\left((0, T)^{k}\right)}^{q}\right]\right\}^{1 / q} .
$$

Also, $\mathbb{D}^{\infty}:=\cap_{q \geq 1} \cap_{j \geq 1} \mathbb{D}^{j, q}$. In the sequel we agree to denote for all $q \geq 1,\|F\|_{q}:=\mathbb{E}\left[|F|^{q}\right]^{1 / q}$.

Skorohod Integral.

We denote by $\mathcal{P}$ the space of simple processes, that is the subspace of $L^{2}([0, T] \times \Omega, \mathscr{F} \times$ $\mathcal{B}([0, T]), d t \otimes d \mathbb{P})$ consisting of $\mathbb{R}^{n}$ valued processes processes $\left(u_{t}\right)_{t \in[0, T]}$ that can be written

$$
u_{t}=\sum_{i=1}^{m} F_{i}\left(W\left(h_{1}\right), \cdots, W\left(h_{m}\right)\right) h_{i}(t)
$$

for some $m \in \mathbb{N}$, where the $\left(F_{i}\right)_{i \in \llbracket 1, m \rrbracket}$ are smooth real valued functions with polynomial growth, $\forall i \in \llbracket 1, m \rrbracket, h_{i} \in L^{2}\left([0, T], \mathbb{R}^{n}\right)$ so that in particular $F_{i}\left(W\left(h_{1}\right), \cdots, W\left(h_{m}\right)\right) \in \mathcal{S}$.

Observe also that with previous definition of the Malliavin derivative for $F \in \mathcal{S}$ we have $\left(D_{s} F\right)_{s \in[0, T]} \in \mathcal{P}$. For $u \in \mathcal{P}$ we define the Skorohod integral

$$
\delta(u):=\sum_{i=1}^{m}\left\{F_{i}\left(W\left(h_{1}\right), \cdots, W\left(h_{m}\right)\right) W\left(h_{i}\right)-\sum_{j=1}^{m} \partial_{j} F_{i}\left(W\left(h_{1}\right), \cdots, W\left(h_{m}\right)\right)\left\langle h_{i}, h_{j}\right\rangle_{L^{2}([0, T]}\right\},
$$

so that in particular $\delta(u) \in \mathcal{S}$. The Skorohod integral is also closable. Its domain writes $\operatorname{Dom}(\delta):=\left\{u \in L^{2}([0, T] \times \Omega): \exists\left(u_{n}\right)_{n \in \mathcal{P}}, u_{n} \underset{n}{\stackrel{L^{2}([0, T] \times \Omega)}{\longrightarrow}} u, \delta\left(u_{n}\right) \underset{n}{\stackrel{L^{2}(\Omega)}{\longrightarrow}} F:=\delta(u)\right\}$.

Ornstein Uhlenbeck operator.

To state the main tool used in our proofs, i.e. the integration by parts formula in its whole generality, we need to introduce a last operator. Namely, the Ornstein-Uhlenbeck operator $L$ which for $F \in \mathcal{S}$ writes:

$$
\begin{aligned}
L F & :=\delta(D F)=\langle\nabla f(W(h)), W(h)\rangle-\operatorname{Tr}\left(D^{2} f(W(h))\left\langle h, h^{*}\right\rangle_{L^{2}(0, T)}\right), \\
W(h) & =\left(W\left(h_{1}\right), \cdots, W\left(h_{m}\right)\right) .
\end{aligned}
$$

This operator is also closable and $\mathbb{D}^{\infty}$ is included in its domain $\operatorname{Dom}(L)$.

Integration by parts.

Proposition 3.1 (Integration by parts: first version) Let $F \in \mathbb{D}^{1,2}, u \in \operatorname{Dom}(\delta)$, then the following indentity holds:

$$
\mathbb{E}\left[\langle D F, u\rangle_{L^{2}([0, T])}\right]=\mathbb{E}[F \delta(u)]
$$


that is the Skorohod integral $\delta$ is the adjoint of the Malliavin derivative D. As a consequence, for $F, G \in \operatorname{Dom}(L)$ we have

$$
\mathbb{E}[F L G]=\mathbb{E}[F \delta(D G)]=\mathbb{E}\left[\langle D F, D G\rangle_{L^{2}([0, T])}\right]=\mathbb{E}[L F G],
$$

i.e. $L$ is self-adjoint.

These relations can be easily checked for $F, G \in \mathcal{S}, u \in \mathcal{P}$, and extended to the indicated domains thanks to the closability.

\subsection{Chaos Decomposition}

$$
I_{m}\left(f_{m}\right):=m ! \int_{0}^{T} \int_{0}^{t_{1}} \cdots \int_{0}^{t_{m}-1} f_{m}\left(t_{1}, \cdots, t_{m}\right) \otimes d W_{t_{m}} \otimes \cdots \otimes d W_{t_{1}} .
$$

In the above equation $\otimes$ denotes the tensor product and $\left(d W_{t_{m}} \otimes \cdots \otimes d W_{t_{1}}\right) \in\left(\left(\mathbb{R}^{n}\right)^{\otimes m}\right)^{*}$.

We now state a theorem that provides a decomposition of real-valued square-integrable random variables in terms of series of multiple integrals.

Lemma 3.2 Let $F$ be a real-valued random variable in $L^{2}(\Omega, \mathscr{F}, \mathbb{P})$. There exists a sequence $\left(f_{m}\right)_{m \in \mathbb{N}}$ s.t.

$$
F=\sum_{m \in \mathbb{N}} I_{m}\left(f_{m}\right)
$$

where for all $m \in \mathbb{N}, f_{m}$ is a symmetric function in $L^{2}\left([0, T]^{m},\left(\mathbb{R}^{n}\right)^{\otimes m}\right)$ and

$$
\mathbb{E}\left[F^{2}\right]=\sum_{m \geq 0} m !\left\|f_{m}\right\|_{L^{2}\left([0, T]^{m},\left(\mathbb{R}^{n}\right)^{\otimes m}\right)}^{2}<+\infty .
$$

We refer to Theorem 1.1.2 in Nualart [32] for a proof.

Remark 3.3 We use the term chaos decomposition for the previous expansion because the multiple integral $I_{m}$ maps $L^{2}\left([0, T]^{m},\left(\mathbb{R}^{n}\right)^{\otimes m}\right)$ onto the Wiener chaos $\mathcal{H}_{m}:=\left\{H_{m}(W(h)), h \in\right.$ $\left.L^{2}\left([0, T], \mathbb{R}^{n}\right),\|h\|_{L^{2}\left([0, T], \mathbb{R}^{n}\right)}=1\right\}$, where $H_{m}$ stands for the Hermite polynomial of degree $m$ (see again Theorem 1.1.2 in [32]). The orthogonality of the Hermite polynomials yields the orthogonality of the Wiener chaos, i.e. $\mathbb{E}[X Y]=0$, for $(X, Y) \in\left(\mathcal{H}_{n}, \mathcal{H}_{m}\right), n \neq m$.

The computation of Malliavin derivatives is quite simple for multiple integrals. Indeed,

$$
D_{t}\left(I_{m}\left(f_{m}\right)\right)=m I_{m-1}\left(f_{m}(t, .)\right) \in \mathbb{R}^{n} .
$$

As a consequence, for a random variable $F$ having a decomposition as in (3.1), we have that it belongs to $\mathbb{D}^{1,2}$ if and only if $\sum_{m \geq 1} m m !\left\|f_{m}\right\|_{L^{2}\left([0, T]^{m},\left(\mathbb{R}^{n}\right)^{\otimes m}\right)}^{2}<+\infty$ in which case $D_{t} F=\sum_{m \geq 1} m I_{m-1}\left(f_{m}(t,).\right)$ and $\mathbb{E}\left[\int_{0}^{T}\left|D_{t} F\right|^{2} d t\right]=\sum_{m \geq 1} m m !\left\|f_{m}\right\|_{L^{2}\left([0, T]^{m},\left(\mathbb{R}^{n}\right)^{\otimes m}\right)}^{2}$. Iterating 
the procedure, one gets $F \in \mathbb{D}^{N, 2} \Longleftrightarrow \sum_{m=N}^{+\infty} \frac{(m !)^{2}}{(m-N) !}\left\|f_{m}\right\|_{L^{2}\left([0, T]^{m},\left(\mathbb{R}^{n}\right)^{\otimes m}\right)}^{2}<+\infty$ and $D_{t_{1}, \cdots, t_{N}} F=\sum_{m=N}^{+\infty} m(m-1) \cdots(m-N+1) I_{m-N}\left(f_{m}\left(t_{1}, \cdots, t_{n},.\right)\right) \in\left(\mathbb{R}^{n}\right)^{\otimes N}$.

Therefore, when a random variable is smooth in the Malliavin sense, i.e. $\mathbb{D}^{\infty}$, the Stroock formula, see [38], provides a representation for the functions $\left(f_{m}\right)_{m \in \mathbb{N}}$ in the chaotic expansion in terms of Malliavin derivatives.

Proposition 3.4 (Stroock's formula) Let $F \in \mathbb{D}^{\infty}$, then the explicit expression of the functions $\left(f_{m}\right)_{m \geq 1}$ in the chaotic expansion (3.1) of $F$ writes:

$$
\forall m \in \mathbb{N}, f_{m}\left(t_{1}, \cdots, t_{m}\right)=\mathbb{E}\left[D_{t_{1}, \cdots, t_{m}}^{m} F\right] \in\left(\mathbb{R}^{n}\right)^{\otimes m} .
$$

For square integrable process, a result analogous to Lemma 3.2 also holds.

Lemma 3.5 Let $\left(u_{t}\right)_{t \in[0, T]}$ be an $\mathbb{R}^{n}$-valued process in $L^{2}([0, T] \times \Omega, \mathscr{F} \times \mathcal{B}([0, T]), d t \otimes d \mathbb{P})$. There exists a sequence of deterministic functions $\left(g_{m}\right)_{m \in \mathbb{N}^{*}}$ s.t.

$$
u_{t}=\sum_{m \geq 0} I_{m}\left(g_{m+1}(t, .)\right)
$$

where the square integrable kernels $g_{m+1}$ are defined on $[0, T]^{m+1}$ with values in $\left(\mathbb{R}^{n}\right)^{\otimes(m+1)}$, are symmetric in the last $m$ variables and s.t. $\sum_{m \geq 0} m !\left\|g_{m+1}\right\|_{L^{2}\left([0, T]^{m+1},\left(\mathbb{R}^{n}\right)^{\otimes(m+1)}\right)}^{2}<+\infty$.

We refer to Lemma 1.3.1 in [32] for a proof when $n=1$.

Also, the Skorohod integral of $u \in \operatorname{Dom}(\delta)$ is quite direct to compute from its chaotic decomposition (3.2). Namely,

$$
\delta(u):=\sum_{m \geq 0} I_{m+1}\left(\widetilde{g}_{m}\right),
$$

where $\widetilde{g}_{m}\left(t, t_{1}, \cdots, t_{m}\right):=\frac{1}{m+1}\left[g_{m}\left(t_{1}, \cdots, t_{m}, t\right)+\sum_{i=1}^{m} g_{m}\left(t_{1}, \cdots, t_{i-1}, t, t_{i+1}, \cdots, t_{m}, t_{i}\right)\right]$ is the symmetrization of $g_{m}$ in $[0, T]^{m+1}$.

\subsection{Representation of densities through Malliavin calculus}

For $F=\left(F_{1}, \cdots, F_{N}\right) \in\left(\mathbb{D}^{\infty}\right)^{N}$, we define the Malliavin covariance matrix $\gamma_{F}$ by

$$
\gamma_{F}^{i, j}:=\left\langle D F^{i}, D F^{j}\right\rangle_{L^{2}(0, T)}, \forall(i, j) \in \llbracket 1, N \rrbracket^{2} .
$$

Let us now introduce the non-degeneracy condition

[ND] We say that the random vector $F=\left(F_{1}, \cdots, F_{N}\right)$ satisfies the non degeneracy condition if $\gamma_{F}$ is a.s. invertible and $\operatorname{det}\left(\gamma_{F}\right)^{-1} \in \cap_{q \geq 1} L^{q}(\Omega)$. In the sequel, we denote the inverse of the Malliavin matrix by

$$
\Gamma_{F}:=\gamma_{F}^{-1} .
$$


This non degeneracy condition guarantees the existence of a smooth density, i.e. $C^{\infty}$, for the random variable $F$, see e.g. Corollary 2.1.2 in [32] or Theorem 9.3 in [21].

The following Proposition will be crucial in the derivation of an explicit representation of the density.

Proposition 3.6 (Second integration by parts) Let $F=\left(F_{1}, \cdots, F_{N}\right) \in\left(\mathbb{D}^{\infty}\right)^{N}$ satisfy the nondegeneracy condition [ND]. Then, for all smooth function $\varphi$ with polynomial growth, $G \in \mathbb{D}^{\infty}$ and all multi-index $\alpha$,

$$
\begin{aligned}
\mathbb{E}\left[\partial_{\alpha} \varphi(F) G\right] & =\mathbb{E}\left[\varphi(F) H_{\alpha}(F, G)\right], \\
H_{i}(F, G) & =-\sum_{j=1}^{N}\left\{G\left\langle D \Gamma_{F}^{i j}, D F^{j}\right\rangle_{L^{2}(0, T)}+\Gamma_{F}^{i j}\left\langle D G, D F^{j}\right\rangle_{L^{2}(0, T)}-\Gamma_{F}^{i j} G L F^{j}\right\}, \forall i \in \llbracket 1, N \rrbracket, \\
H_{\alpha}(F, G) & =H_{\left(\alpha_{1}, \cdots, \alpha_{m}\right)}(F, G)=H_{\alpha_{m}}\left(F, H_{\left(\alpha_{1}, \cdots, \alpha_{m-1}\right)}(F, G)\right) .
\end{aligned}
$$

Also, for all $q>1$, and all multi-index $\alpha$, there exists $\left(C, q_{0}, q_{1}, q_{2}, r_{1}, r_{2}\right)$ only depending on $(q, \alpha)$ s.t.

$$
\left\|H_{\alpha}(F, G)\right\|_{q} \leq C\left\|\Gamma_{F}\right\|_{q_{0}}\|G\|_{q_{1}, r_{1}}\|F\|_{q_{2}, r_{2}} .
$$

For the first part of the proposition we refer to Section V-9 of [21]. Concerning equation (3.3), it can be directly derived from the Meyer inequalities on $\|L F\|_{q}$ and the explicit definition of $H$, see also Proposition 2.4 in Bally and Talay [3].

A crucial consequence of the integration by parts formula is the following representation for the density.

Corollary 3.7 (Expression of the density and upper bound) Let $F=\left(F_{1}, \cdots, F_{N}\right) \in$ $\left(\mathbb{D}^{\infty}\right)^{N}$ satisfy the nondegeneracy condition [ND]. The random vector $F$ admits a density on $\mathbb{R}^{N}$. Fix $y \in \mathbb{R}^{N}$. Introduce $\forall(u, v) \in \mathbb{R}^{2}, \varphi_{0}^{u}(v)=\mathbb{I}_{v>u}, \varphi_{1}^{u}(v)=\mathbb{I}_{v \leq u}$. For all multi-index $\beta=\left(\beta_{1}, \cdots, \beta_{N}\right) \in\{0,1\}^{N}$ the density writes:

$$
p_{F}(y)=\mathbb{E}\left[\prod_{i=1}^{N} \varphi_{\beta_{i}}^{y_{i}}\left(F_{i}\right) H_{\alpha}(F, 1)\right](-1)^{|\beta|}, \alpha=(1, \cdots, N),|\beta|:=\sum_{i=1}^{N} \beta_{i} .
$$

As a consequence of (3.4) and (3.3) we get for all multi-index $\beta \in\{0,1\}^{N}$ :

$$
\exists C>0, p_{F}(y) \leq C \prod_{i=1}^{N} \mathbb{E}\left[\varphi_{\beta_{i}}^{y_{i}}\left(F_{i}\right)\right]^{\gamma(i)}\left\|H_{\alpha}(F, 1)\right\|_{2}, \quad \gamma(i)=2^{-(i+1)} .
$$

Proof. Let $B:=\prod_{i=1}^{N}\left[a_{i}, b_{i}\right], \forall i \in \llbracket 1, N \rrbracket, a_{i}<b_{i}$. Denote for all $u \in \mathbb{R}, I_{0}(u):=$ $(-\infty, u), I_{1}(u):=[u, \infty)$. Set finally, for all multi-index $\beta \in\{0,1\}^{N}, \forall y \in \mathbb{R}^{N}, \Psi_{B}^{\beta}(y)=$ $\int_{\prod_{i=1}^{N} I_{\beta_{i}}\left(y_{i}\right)} \mathbb{I}_{B}(x) d x$. Proposition 3.6 applied with $\alpha=(1, \cdots, N)$ and $\Psi_{B}^{\beta}$ yields

$$
\mathbb{E}\left[\partial_{\alpha} \Psi_{B}^{\beta}(F)\right]=\mathbb{E}\left[\Psi_{B}^{\beta}(F) H_{\alpha}(F, 1)\right]
$$


Now, the r.h.s. of equation (3.6) writes

$$
\begin{aligned}
\mathbb{E}\left[\Psi_{B}^{\beta}(F) H_{\alpha}(F, 1)\right] & =\mathbb{E}\left[\int_{\prod_{i=1}^{N} I_{\beta_{i}}\left(F_{i}\right)} \mathbb{I}_{B}(y) d y H_{\alpha}(F, 1)\right]=\int_{B} \mathbb{E}\left[\prod_{i=1}^{N} \mathbb{I}_{y_{i} \in I_{\beta_{i}}\left(F_{i}\right)} H_{\alpha}(F, 1)\right] d y \\
& =\int_{B} \mathbb{E}\left[\prod_{i=1}^{N} \varphi_{\beta_{i}}^{y_{i}}\left(F_{i}\right) H_{\alpha}(F, 1)\right] d y
\end{aligned}
$$

The application of Fubini's theorem for the last but one equality is justified thanks to the integrability condition (3.3) of Proposition 3.6. On the other hand, the l.h.s. in (3.6) writes

$$
\mathbb{E}\left[\partial_{\alpha} \Psi_{B}^{\beta}(F)\right]=\mathbb{E}\left[\prod_{i=1}^{N} \mathbb{I}_{F_{i} \in\left[a_{i}, b_{i}\right]}(-1)^{\beta_{i}}\right]=(-1)^{|\beta|} \int_{B} p_{F}(y) d y .
$$

Equation (3.4) is now a direct consequence of (3.6), (3.7), (3.8). Equation (3.5) is then simply derived applying iteratively the Cauchy-Schwarz inequality.

\section{Malliavin Calculus to Derive Upper and Diagonal Bounds in our Examples}

\subsection{Strategy and usual Brownian controls}

We here concentrate on the particular case of the process (1.3) (indeed the estimates concerning (1.4) can be derived in a similar way). Since condition $[\mathbf{H}]$ is satisfied, assumption [ND] is fullfilled. It then follows from Theorem 2.3.2 in [32] that the process $\left(X_{s}\right)_{s \geq 0}$ admits a smooth density $p(t, x,$.$) at time t>0$. Our goal is to derive quantitative estimates on this density, emphasizing as well that we have different regimes in function of the starting/final points.

To do that, we condition w.r.t. to the non-degenerate Brownian component for which we explicitly know the density. For all $(t, x, \xi) \in \mathbb{R}^{+*} \times\left(\mathbb{R}^{n+1}\right)^{2}$ we have:

$$
\begin{aligned}
& p(t, x, \xi)=p_{X^{1, n}}\left(t, x_{1, n}, \xi_{1, n}\right) p_{X^{n+1}}\left(t, x_{n+1}, \xi_{n+1} \mid X_{0}^{1, n}=x_{1, n}, X_{t}^{1, n}=\xi_{1, n}\right), \\
& p_{X^{1, n}}\left(t, x_{1, n}, \xi_{1, n}\right)=\frac{1}{(2 \pi t)^{n / 2}} \exp \left(-\frac{\left|\xi_{1, n}-x_{1, n}\right|^{2}}{2 t}\right) .
\end{aligned}
$$

We then focus on the conditional density which agrees with the one of a smooth functional, in the Malliavin sense, of the Brownian bridge. Precisely:

$$
\begin{aligned}
& p_{X^{n+1}}\left(t, x_{n+1}, \xi_{n+1} \mid X_{0}^{1, n}=x_{1, n}, X_{t}^{1, n}=\xi_{1, n}\right):=p_{Y_{t}}\left(\xi_{n+1}-x_{n+1}\right), \\
& Y_{t}:=\int_{0}^{t}\left|x_{1, n} \frac{t-u}{t}+\xi_{1, n} \frac{u}{t}+W_{u}^{0, t}\right|^{k} d u
\end{aligned}
$$

where $\left(W_{u}^{0, t}\right)_{u \in[0, t]}$ is the standard $n$-dimensional Brownian bridge on the interval $[0, t]$. The estimation of $p_{Y_{t}}$ is the core of the probabilistic part of the current work. 
We recall, see e.g. [34], two ways to realize the standard $n$-dimensional Brownian bridge from a standard Brownian motion of $\mathbb{R}^{n}$. Namely, if $\left(W_{t}\right)_{t \geq 0}$ denotes a standard $n$-dimensional Brownian motion then

$$
\begin{array}{r}
\left(W_{u}-\frac{u}{t} W_{t}\right)_{u \in[0, t]} \stackrel{(\text { law })}{=}\left(W_{u}^{0, t}\right)_{u \in[0, t]}, \\
\left((t-u) \int_{0}^{u} \frac{d W_{s}}{t-s}\right)_{u \in[0, t]} \stackrel{(\text { law })}{=}\left(W_{u}^{0, t}\right)_{u \in[0, t]} .
\end{array}
$$

To recover the framework of Section 3.2, in order to deal with functionals of the Brownian increments, it is easier to consider the realization of the Brownian bridge given by (4.3).

Remark 4.1 The process $\left(\bar{W}_{u}\right)_{u \in[0, t]}:=\left(W_{t-u}-W_{t}\right)_{u \in[0, t]}$ is a Brownian motion. Moreover, the processes $\left(\bar{W}_{u}-\frac{u}{t} \bar{W}_{t}\right)_{u \in[0, t]}$ and $\left((t-u) \int_{0}^{u} \frac{d \bar{W}_{s}}{t-s}\right)_{u \in[0, t]}$ are standard $n$-dimensional Brownian bridges on $[0, t]$, as well.

For the sake of completeness, we recall some well known results concerning the Brownian motion and Brownian bridge.

Proposition 4.2 Let $q \geq 1$, and $\left(W_{t}\right)_{t \geq 0}$ be a standard $n$-dimensional Brownian motion. Then, there exists $C:=C(q, n)>0$ s.t. for all $t \geq 0$,

$$
\begin{array}{r}
\mathbb{E}\left[\left|W_{t}\right|^{q}\right] \leq C(q, n) t^{q / 2}, \quad \mathbb{E}\left[\sup _{s \in[0, t]}\left|W_{s}\right|^{q}\right] \leq C(q, n) t^{q / 2}, \\
\mathbb{E}\left[\sup _{s \in[\tau, t]}\left|W_{s}^{0, t}\right|^{q}\right] \leq C(q, n)(t-\tau)^{q / 2}, 0 \leq \tau \leq t .
\end{array}
$$

Moreover, there exists $\bar{c}:=\bar{c}(n) \geq 1$, s.t. for all $\zeta \geq 0$, and $0 \leq \tau \leq t$,

$$
\mathbb{P}\left[\sup _{s \in[\tau, t]}\left|W_{s}^{0, t}\right| \geq \zeta\right] \leq 2 \exp \left(-\frac{|\zeta|^{2}}{\bar{c}(n)(t-\tau)}\right) .
$$

Proof. The first inequality is a simple consequence of the Brownian scaling. The second one can be derived from convexity inequalities and Lévy's identity that we now recall (see e.g. Chapter 6 in [34]). Let $\left(B_{t}\right)_{t \geq 0}$ be a standard scalar Brownian motion. Then:

$$
\sup _{u \in[0, s]} B_{u} \stackrel{(\text { law })}{=}\left|B_{s}\right|, \forall s>0
$$

The third inequality follows from the first two and the representation (4.2). Eventually, the deviation estimates follow from (4.4) as well. These deviations estimates can also be seen as special cases of Bernstein's inequality, see e.g. [34] p. 153.

\subsection{Some preliminary estimates on the Malliavin derivative and covariance matrix}

We now give the expressions of the Malliavin derivative and covariance matrix of the scalar random variable $Y_{t}$ defined in (4.1) and some associated controls. 
Lemma 4.3 (Malliavin Derivative and some associated bounds) Let us set $m\left(u, t, x_{1, n}, \xi_{1, n}\right):=x_{1, n} \frac{t-u}{t}+\xi_{1, n} \frac{u}{t}$. Rewrite

$$
\begin{aligned}
Y_{t} & =\int_{0}^{t} d u\left|m\left(u, t, x_{1, n}, \xi_{1, n}\right)+W_{u}^{0, t}\right|^{k} \\
& =\int_{0}^{t} d u\left\{\left|m\left(u, t, x_{1, n}, \xi_{1, n}\right)\right|^{2}+\left|W_{u}^{0, t}\right|^{2}+2\left\langle m\left(u, t, x_{1, n}, \xi_{1, n}\right), W_{u}^{0, t}\right\rangle\right\}^{k / 2} \\
& =\sum_{i=0}^{k / 2} C_{k / 2}^{i} \int_{0}^{t} d u\left|m\left(u, t, x_{1, n}, \xi_{1, n}\right)\right|^{k-2 i}\left\{\left|W_{u}^{0, t}\right|^{2}+2\left\langle m\left(u, t, x_{1, n}, \xi_{1, n}\right), W_{u}^{0, t}\right\rangle\right\}^{i} .
\end{aligned}
$$

Considering the realization (4.3) of the Brownian bridge, the Malliavin derivative of $Y_{t}$ (seen as a column vector) and the "covariance" matrix (that is in our case a scalar) write for all $s \in[0, t]:$

$$
\begin{aligned}
D_{s} Y_{t}= & \sum_{i=1}^{k / 2} C_{k / 2}^{i} \int_{s}^{t} d u\left|m\left(u, t, x_{1, n}, \xi_{1, n}\right)\right|^{k-2 i} i\left\{\left|W_{u}^{0, t}\right|^{2}+2\left\langle m\left(u, t, x_{1, n}, \xi_{1, n}\right), W_{u}^{0, t}\right\rangle\right\}^{i-1} \\
& \times 2 \frac{t-u}{t-s}\left(W_{u}^{0, t}+m\left(u, t, x_{1, n}, \xi_{1, n}\right)\right):=\sum_{i=1}^{k / 2} M_{i}\left(s, t, x_{1, n}, \xi_{1, n}\right) \\
\gamma_{Y_{t}}= & \int_{0}^{t} d s\left|D_{s} Y_{t}\right|^{2} .
\end{aligned}
$$

Introduce now

$$
\begin{aligned}
M_{1}\left(s, t, x_{1, n}, \xi_{1, n}\right):= & k \int_{s}^{t} d u\left|m\left(u, t, x_{1, n}, \xi_{1, n}\right)\right|^{k-2} \frac{t-u}{t-s} m\left(u, t, x_{1, n}, \xi_{1, n}\right) \\
& +M_{1}^{R}\left(s, t, x_{1, n}, \xi_{1, n}\right):=\left(M_{1}^{D}+M_{1}^{R}\right)\left(s, t, x_{1, n}, \xi_{1, n}\right), \\
R\left(s, t, x_{1, n}, \xi_{1, n}\right):= & M_{1}^{R}\left(s, t, x_{1, n}, \xi_{1, n}\right)+\sum_{i=2}^{k / 2} M_{i}\left(s, t, x_{1, n}, \xi_{1, n}\right), \\
\gamma_{Y_{t}}= & \int_{0}^{t} d s\left|\left(M_{1}^{D}+R\right)\left(s, t, x_{1, n}, \xi_{1, n}\right)\right|^{2} .
\end{aligned}
$$

Set for all $\tau \in[0, t]$,

$$
\begin{aligned}
\mathcal{M}_{\tau, t} & :=\int_{\tau}^{t} d s\left|M_{1}^{D}\left(s, t, \xi_{1, n}, \xi_{1, n}\right)\right|^{2}, \quad \mathcal{M}_{t}:=\mathcal{M}_{0, t}, \\
\mathcal{R}_{\tau, t} & :=\int_{\tau}^{t} d s\left|R\left(s, t, \xi_{1, n}, \xi_{1, n}\right)\right|^{2}, \quad \mathcal{R}_{t}:=\mathcal{R}_{0, t} .
\end{aligned}
$$

There exists $C:=C(k, n) \geq 1$ s.t. for all $\tau \in[0, t]$ :

$$
C^{-1}(t-\tau)^{3}\left(\left|x_{1, n}\right|^{2(k-1)}+\left|\xi_{1, n}\right|^{2(k-1)}\right) \leq \mathcal{M}_{\tau, t} \leq C(t-\tau)^{3}\left(\left|x_{1, n}\right|^{2(k-1)}+\left|\xi_{1, n}\right|^{2(k-1)}\right) .
$$


Also, for all $q \geq 1$, there exists $C(k, n, q)$ s.t.

$$
\begin{aligned}
\mathbb{E}\left[\left|\mathcal{R}_{\tau, t}\right|^{q}\right]^{1 / q} \leq & C(k, n, q)(t-\tau)^{3}\left(\left|x_{1, n}\right| \vee\left|\xi_{1, n}\right|\right)^{2(k-1)} \\
& \times \frac{(t-\tau)}{\left(\left|x_{1, n}\right| \vee\left|\xi_{1, n}\right|\right)^{2}}\left(1+\frac{(t-\tau)^{1 / 2}}{\left|x_{1, n}\right| \vee\left|\xi_{1, n}\right|}\right)^{2(k-2)}, \\
\forall \kappa \geq 0, \quad \mathbb{P}\left[\mathcal{R}_{\tau, t} \geq \kappa \mathcal{M}_{\tau, t}\right] \leq & \bar{c}(n, k) \exp \left(-\kappa^{2} \frac{\left(\left|\xi_{1, n}\right| \vee\left|x_{1, n}\right|\right)^{2}}{\bar{c}(n, k)(t-\tau)}\right),
\end{aligned}
$$

for some constant $\bar{c}(n, k) \geq 1$.

Remark 4.4 From (4.10) and (4.11), it follows that

$$
\mathbb{E}\left[\mathcal{R}_{t}^{q}\right]^{1 / q} \leq \frac{C(k, n, q) C}{K^{2}}\left(1+\frac{1}{K}\right)^{2(k-2)} \mathcal{M}_{t},
$$

when $\left|x_{1, n}\right| \vee\left|\xi_{1, n}\right| \geq K t^{1 / 2}$. For $K:=K(k, n, q)$ large enough, then the term $\mathcal{M}_{t}$ (corresponding to the Malliavin covariance matrix of a Gaussian contribution) dominates the remainder. This intuitively explains the Gaussian regime appearing in ii) of Theorem 2.1.

Proof. Assertion (4.6) directly follows from the chain rule (see e.g. Proposition 1.2.3 in [32]) and the identity $D_{s} W_{u}^{0, t}=\mathbb{I}_{s \leq u} \frac{t-u}{t-s}, \forall(u, s) \in[0, t]^{2}$ deriving from (4.3).

Concerning (4.10), we only prove the claim for $\tau=0$ for notational simplicity. Usual computations involving convexity inequalities yield that there exists $C:=C(k, n) \geq 1$ s.t.

$$
\mathcal{M}_{t} \leq C t^{3}\left(\left|x_{1, n}\right|^{2(k-1)}+\left|\xi_{1, n}\right|^{2(k-1)}\right) .
$$

On the other hand to prove that a lower bound at the same ordre also holds for $\mathcal{M}_{t}$ one has to be a little more careful.

W.l.o.g. we can assume that $\left|\xi_{1, n}\right| \geq\left|x_{1, n}\right|$. Indeed, because of the symmetry of the Brownian Bridge and its reversibility in time (see Remark 4.1), if $\left|\xi_{1, n}\right|<\left|x_{1, n}\right|$ we can perform the computations w.r.t. to the Brownian bridge $\left(\bar{W}_{u}^{0, t}\right)_{u \in[0, t]}:=\left(W_{t-u}^{0, t}\right)_{u \in[0, t]}$ using the sensitivity w.r.t. to the Brownian motion $\left(\bar{W}_{u}\right)_{u \in[0, t]}:=\left(W_{t-u}-W_{t}\right)_{u \in[0, t]}$. Note that $\left|\xi_{1, n}\right| \geq\left|x_{1, n}\right| \Rightarrow\left|\xi_{1, n}\right|_{\infty} \geq \frac{1}{n^{1 / 2}}\left|x_{1, n}\right|_{\infty}$. Let $i_{0} \in \llbracket 1, n \rrbracket$ be the index s.t. $\left|\xi_{1, n}\right|_{\infty}:=\left|\xi_{i_{0}}\right|$, then $\left|\xi_{i_{0}}\right| \geq \frac{1}{n^{1 / 2}}\left|x_{i_{0}}\right|$. Let us now write

$$
\mathcal{M}_{t} \geq k^{2} \int_{0}^{t} d s\left(\int_{s}^{t} d u\left|m\left(u, t, x_{1, n}, \xi_{1, n}\right)\right|^{k-2}\left(\frac{t-u}{t} x_{i_{0}}+\frac{u}{t} \xi_{i_{0}}\right) \frac{t-u}{t-s}\right)^{2} .
$$

Observe now that for $s \geq \frac{n^{1 / 2}}{n^{1 / 2}+1} t$ we have that $\forall u \in[s, t], \frac{t-u}{t} x_{i_{0}}+\frac{u}{t} \xi_{i_{0}}$ has the sign of $\xi_{i_{0}}$. Hence,

$$
\mathcal{M}_{t} \geq k^{2} \int_{\frac{n^{1 / 2}}{n^{1 / 2}+1}}^{t} d s\left(\int_{s}^{t} d u\left|\frac{t-u}{t} x_{i_{0}}+\frac{u}{t} \xi_{i_{0}}\right|^{k-1} \frac{t-u}{t-s}\right)^{2}
$$


Now, for $s \geq t\left(\frac{n^{1 / 2}}{n^{1 / 2}+2^{-1}}\right)$, we have for all $u \in[s, t]$ :

$$
\begin{aligned}
\left|\frac{t-u}{t} x_{i_{0}}+\frac{u}{t} \xi_{i_{0}}\right|^{k-1} & \geq\left(\frac{u}{t}\right)^{k-1} \frac{\left|\xi_{i_{0}}\right|^{k-1}}{2^{k-2}}-\left(\frac{t-u}{t}\right)^{k-1}\left|x_{i_{0}}\right|^{k-1} \\
& \geq\left|\xi_{i_{0}}\right|^{k-1}\left(\frac{n^{1 / 2}}{2 n^{1 / 2}+1}\right)^{k-1} .
\end{aligned}
$$

Equation (4.10) thus follows from (4.14), (4.15) and (4.13).

Concerning the remainders we get that there exists $C_{3}:=C_{3}(n, k), C_{4}:=C_{4}(n, k)$ s.t.:

$$
\begin{aligned}
\left|M_{1}^{R}\left(s, t, x_{1, n}, \xi_{1, n}\right)\right|^{2} \leq & C_{3}(t-s)^{2}\left|\xi_{1, n}\right|^{2(k-1)} \sup _{u \in[s, t]}\left|W_{u}^{0, t}\right|^{2}\left|\xi_{1, n}\right|^{-2} \\
\forall i \in \llbracket 2, k / 2 \rrbracket,\left|M_{i}\left(s, t, x_{1, n}, \xi_{1, n}\right)\right|^{2} \leq & C_{4}(t-s)^{2}\left|\xi_{1, n}\right|^{2(k-1)}\left\{\sup _{u \in[s, t]}\left|W_{u}^{0, t}\right|^{2(2 i-1)}\left|\xi_{1, n}\right|^{-2(2 i-1)}\right. \\
& \left.+\sup _{u \in[s, t]}\left|W_{u}^{0, t}\right|^{2(i-1)}\left|\xi_{1, n}\right|^{-2(i-1)}\right\}
\end{aligned}
$$

From (4.8) and a convexity inequality, we derive $\left|R\left(s, t, x_{1, n}, \xi_{1, n}\right)\right|^{2} \leq \frac{k}{2}\left(\left|M_{1}^{R}\left(s, t, x_{1, n}, \xi_{1, n}\right)\right|^{2}+\right.$ $\left.\sum_{i=2}^{k / 2}\left|M_{i}\left(s, t, x_{1, n}, \xi_{1, n}\right)\right|^{2}\right)$. Thus, from (4.16), (4.9), we obtain that there exists $C(k, n, q)$ s.t. for all $\tau \in[0, t]$ :

$$
\begin{aligned}
\mathbb{E}\left[\left|\mathcal{R}_{\tau, t}\right|^{q}\right]^{1 / q} \leq & C(k, n, q)(t-\tau)^{3}\left|\xi_{1, n}\right|^{2(k-1)}\left\{\sum_{i=1}^{k / 2} \mathbb{E}\left[\left.\left.\left|\sup _{u \in[\tau, t]}\right| W_{u}^{0, t}\right|^{2(2 i-1)}\left|\xi_{1, n}\right|^{-2(2 i-1)}\right|^{q}\right]^{1 / q}\right. \\
& \left.+\sum_{i=2}^{k / 2} \mathbb{E}\left[\left.\left.\left|\sup _{u \in[\tau, t]}\right| W_{u}^{0, t}\right|^{2(i-1)}\left|\xi_{1, n}\right|^{-2(i-1)}\right|^{q}\right]^{1 / q}\right\}
\end{aligned}
$$

which, thanks to Proposition 4.2, gives (4.11). 
On the other hand, from (4.10) and the previous convexity inequality for $R$ we get:

$$
\begin{aligned}
& \mathbb{P}\left[\kappa \mathcal{M}_{\tau, t} \leq \mathcal{R}_{\tau, t}\right] \leq \mathbb{P}\left[C^{-1}(t-\tau)^{3}\left|\xi_{1, n}\right|^{2(k-1)} \kappa \leq \frac{k}{2}\left[\int_{\tau}^{t} d s\left|M_{1}^{R}\left(s, t, x_{1, n}, \xi_{1, n}\right)\right|^{2}\right.\right. \\
+ & \left.\left.\sum_{i=2}^{k / 2} \int_{\tau}^{t} d s\left|M_{i}\left(s, t, x_{1, n}, \xi_{1, n}\right)\right|^{2}\right]\right] \\
\leq \quad & \mathbb{P}\left[\left(\frac{2}{k}\right)^{2} C^{-1}(t-\tau)^{3}\left|\xi_{1, n}\right|^{2(k-1)} \kappa \leq \int_{\tau}^{t} d s\left|M_{1}^{R}\left(s, t, x_{1, n}, \xi_{1, n}\right)\right|^{2}\right] \\
+ & +\sum_{i=2}^{k / 2} \mathbb{P}\left[\left(\frac{2}{k}\right)^{2} C^{-1}(t-\tau)^{3}\left|\xi_{1, n}\right|^{2(k-1)} \kappa \leq \int_{\tau}^{t} d s\left|M_{i}\left(s, t, x_{1, n}, \xi_{1, n}\right)\right|^{2}\right] \\
\underset{(4.16)}{\leq} & \mathbb{P}\left[\left(\frac{2}{k}\right)^{2} C^{-1}(t-\tau)^{3}\left|\xi_{1, n}\right|^{2(k-1)} \kappa \leq \frac{C_{3}}{3}(t-\tau)^{3}\left|\xi_{1, n}\right|^{2(k-1)} \sup _{u \in[\tau, t]}\left|W_{u}^{0, t}\right|^{2}\left|\xi_{1, n}\right|^{-2}\right] \\
+ & \sum_{i=2}^{k / 2} \mathbb{P}\left[\left(\frac{2}{k}\right)^{2} C^{-1} \kappa \leq \frac{C_{4}}{3}\left\{\left(\frac{\sup _{u \in[\tau, t]}\left|W_{u}^{0, t}\right|}{\left|\xi_{1, n}\right|}\right)^{2(2 i-1)}+\left(\frac{\sup _{u \in[\tau, t]}\left|W_{u}^{0, t}\right|}{\left|\xi_{1, n}\right|}\right)^{2(i-1)}\right\}\right] .
\end{aligned}
$$

Equation (4.12) then follows from Proposition 4.2.

\subsection{Control of the weights}

Now to exploit Corollary 3.7 to give estimates on $p_{Y_{t}}$ we need to have bounds on the Malliavin weights. Formula (3.4) involves two kinds of terms: the inverse of the Malliavin Matrix and the Ornstein-Uhlenbeck operator. Lemma 4.3 provides tools to analyze the Malliavin matrix. Concerning the Ornstein-Uhlenbeck operator we will rely on the chaos expansion techniques introduced in Section 3.3.

\subsection{1 "Gaussian" regime}

In this section we assume that $\left|x_{1, n}\right| \vee\left|\xi_{1, n}\right| \geq K t^{1 / 2}$, for $K:=K(n, d)$ sufficiently large. That is we suppose that the starting or the final point of the non-degenerate component has greater norm than the characteristic time-scale $t^{1 / 2}$. In this case, we show below that the dominating term in the Malliavin derivative is the one associated to the non-random term $M_{1}^{D}$ in (4.7). This term corresponds to the Malliavin derivative of a Gaussian process. This justifies the terminology "Gaussian" regime.

In order to give precise asymptotics on the density of $Y_{t}$, the crucial step consists in controlling the norm of $\Gamma_{Y_{t}}:=\gamma_{Y_{t}}^{-1}$ in $L^{q}(\Omega), q \in[1,+\infty)$ spaces.

Lemma 4.5 (Estimates on the Malliavin covariance) Assume that $\left|x_{1, n}\right| \vee\left|\xi_{1, n}\right| \geq K t^{1 / 2}$. Then, for all $q \in[1,+\infty)$ there exists $C_{q, 4.5}:=C_{q, 4.5}(n, k, K) \geq 1$ s.t.

$$
\frac{C_{q, 4.5}^{-1}}{\left(\left|x_{1, n}\right|^{2(k-1)}+\left|\xi_{1, n}\right|^{2(k-1)}\right) t^{3}} \leq\left\|\Gamma_{Y_{t}}\right\|_{q} \leq \frac{C_{q, 4.5}}{\left(\left|x_{1, n}\right|^{2(k-1)}+\left|\xi_{1, n}\right|^{2(k-1)}\right) t^{3}} .
$$


Proof. As in Lemma 4.3, we assume, without loss of generality, that $\left|\xi_{1, n}\right| \geq\left|x_{1, n}\right|$. To give the $L^{q}$ estimates of the Malliavin derivative we recall the definition of $\mathcal{M}_{t}$ given in (4.9), and we use the following partition:

$$
\begin{gathered}
\mathbb{E}\left[\left|\Gamma_{Y_{t}}\right|^{q}\right]=\sum_{m \in \mathbb{N}} \mathbb{E}\left[\left|\Gamma_{Y_{t}}\right|^{q} \mathbb{I}_{\Gamma_{Y_{t}} \in\left[\frac{4 m}{\mathcal{M}_{t}}, \frac{4(m+1)}{\mathcal{M}_{t}}\right]}\right] \leq \\
\left(\frac{4}{\mathcal{M}_{t}}\right)^{q}+\sum_{m \geq 1}\left(\frac{4(m+1)}{\mathcal{M}_{t}}\right)^{q} \mathbb{P}\left[\gamma_{Y_{t}} \leq \frac{\mathcal{M}_{t}}{4 m}\right] .
\end{gathered}
$$

Equation (4.10) in Lemma 4.3 provides us with an useful bound for $\mathcal{M}_{t}$. We next give estimates of $\mathbb{P}\left[\gamma_{Y_{t}} \leq \frac{\mathcal{M}_{t}}{4 m}\right], m \geq 1$ in the spirit of Bally [1].

Introduce $t_{m}:=\inf \left\{v \in[0, t]: \mathcal{M}_{v, t} \leq \mathcal{M}_{t} / m\right\}$. We first show that there exists $m_{0} \in \mathbb{N}$ and $\bar{C}:=\bar{C}(n, k)$ such that $t_{m} \geq t\left(1-\bar{C} m^{-1 / 3}\right)$ for all $m \geq m_{0}$. From (4.10) we obtain

$$
\begin{aligned}
t_{m} & \geq \inf \left\{v \in[0, t]: \mathcal{M}_{v, t} \leq C t^{3}\left(\left|x_{1, n}\right|^{2(k-1)}+\left|\xi_{1, n}\right|^{2(k-1)}\right) / m\right\} \\
& \geq \inf \left\{v \in[0, t]: \mathcal{M}_{v, t} \leq 2 C t^{3}\left|\xi_{1, n}\right|^{2(k-1)} / m\right\}=: \bar{t}_{m},
\end{aligned}
$$

recalling we have assumed $\left|\xi_{1, n}\right| \geq\left|x_{1, n}\right|$ for the last inequality. Equations (4.14) and (4.15) also yield that there exists $C_{2}:=C_{2}(n, k)$ s.t. for all $v \geq \frac{n^{1 / 2}}{n^{1 / 2}+2^{-1}} t$,

$$
\mathcal{M}_{v, t} \geq C_{2}(t-v)^{3}\left|\xi_{1, n}\right|^{2(k-1)} \text {. }
$$

Note that $\bar{t}_{m} \rightarrow t$ as $m \rightarrow+\infty$, then there exists $\bar{m}$ such that $\bar{t}_{m} \geq \frac{n^{1 / 2}}{n^{1 / 2}+2^{-1}} t$ for every $m \geq \bar{m}$, and the above inequality holds for every $v \in\left[\bar{t}_{m}, t\right]$. Set $\bar{C}:=\left(2 C / C_{2}\right)^{1 / 3}$, and $m_{0}=\left\lfloor\bar{C}^{3}\right\rfloor \vee \bar{m}$. For every $m \geq m_{0}$ we have that:

$$
\begin{aligned}
\mathbb{P}\left[\gamma_{Y_{t}} \leq \frac{\mathcal{M}_{t}}{4 m}\right] & \leq \mathbb{P}\left[\int_{t_{m}}^{t} d s\left|\left(M_{1}^{D}+R\right)\left(s, t, x_{1, n}, \xi_{1, n}\right)\right|^{2} \leq \frac{\mathcal{M}_{t}}{4 m}\right] \\
& \leq \mathbb{P}\left[\frac{1}{2} \int_{t_{m}}^{t} d s\left|M_{1}^{D}\left(s, t, x_{1, n}, \xi_{1, n}\right)\right|^{2}-\int_{t_{m}}^{t} d s\left|R\left(s, t, x_{1, n}, \xi_{1, n}\right)\right|^{2} \leq \frac{\mathcal{M}_{t}}{4 m}\right] \\
& \leq \mathbb{P}\left[\frac{\mathcal{M}_{t_{m}, t}}{4} \leq \mathcal{R}_{t_{m}, t}\right] \leq \bar{c}(n, k) \exp \left(-\frac{\left|\xi_{1, n}\right|^{2} m^{1 / 3}}{16 \bar{C} \bar{c}(n, k) t}\right)
\end{aligned}
$$

using (4.12) for the last inequality. Plugging this control into (4.17), using once again (4.10) 
we derive that there exists $C_{3}:=C_{3}(n, k),\left(C_{4}, C_{5}\right):=\left(C_{4}, C_{5}\right)(n, k, q)$ s.t.:

$$
\begin{array}{r}
\mathbb{E}\left[\mid \Gamma_{\left.Y_{t}\right|^{q}}\right] \leq\left(\frac{4 C m_{0}^{2}}{t^{3}\left|\xi_{1, n}\right|^{2(k-1)}}\right)^{q}+C_{3} \sum_{m \geq m_{0}}\left(\frac{4 C(m+1)}{t^{3}\left|\xi_{1, n}\right|^{2(k-1)}}\right)^{q} \exp \left(-C_{3}^{-1} \frac{\left|\xi_{1, n}\right|^{2} m^{1 / 3}}{t}\right) \\
\leq\left(\frac{4 C m_{0}^{2}}{t^{3}\left|\xi_{1, n}\right|^{2(k-1)}}\right)^{q}+\left(\frac{C_{3}(8 C)^{q}}{\left|\xi_{1, n}\right|^{(2(k-1)+6) q}}\right) \sum_{m \geq m_{0}}\left(\frac{m^{1 / 3}\left|\xi_{1, n}\right|^{2}}{t}\right)^{3 q} \exp \left(-C_{3}^{-1} \frac{\left|\xi_{1, n}\right|^{2} m^{1 / 3}}{t}\right) \\
\leq\left(\frac{4 C m_{0}^{2}}{t^{3}\left|\xi_{1, n}\right|^{2(k-1)}}\right)^{q}+\frac{C_{4}}{\left|\xi_{1, n}\right|^{(2 k+4) q}} \sum_{m \geq m_{0}} \exp \left(-C_{4}^{-1} \frac{\left|\xi_{1, n}\right|^{2} m^{1 / 3}}{t}\right) \\
\leq C_{5}\left[\frac{1}{t^{3 q}\left|\xi_{1, n}\right|^{2 q(k-1)}}+\frac{1}{\left|\xi_{1, n}\right|^{(2 k+4) q}} \frac{t^{3}}{\left|\xi_{1, n}\right|^{6}}\right] \leq \frac{C_{5}}{t^{3 q}\left|\xi_{1, n}\right|^{2 q(k-1)}}\left[1+\frac{t^{3(q+1)}}{\left|\xi_{1, n}\right|^{6(q+1)}}\right]
\end{array}
$$

which for $\left|\xi_{1, n}\right| \geq K t^{1 / 2}$ gives the upper bound of the lemma.

Let us now turn to the lower bound for $\left\|\Gamma_{Y_{t}}\right\|_{L^{p}(\mathbb{P})}$. Write:

$$
\mathbb{E}\left[\Gamma_{Y_{t}}^{q}\right] \geq \mathbb{E}\left[\Gamma_{Y_{t}}^{q} \mathbb{I}_{\gamma_{Y_{t}} \leq 3 \mathcal{M}_{t}}\right] \geq \frac{1}{\left(3 \mathcal{M}_{t}\right)^{q}} \mathbb{P}\left[\gamma_{Y_{t}} \leq 3 \mathcal{M}_{t}\right] \geq \frac{1}{\left(3 \mathcal{M}_{t}\right)^{q}}\left(1-\mathbb{P}\left[\gamma_{Y_{t}}>3 \mathcal{M}_{t}\right]\right) .
$$

From equations (4.6)-(4.8) one has $\mathbb{P}\left[\gamma_{Y_{t}}>3 \mathcal{M}_{t}\right] \leq \mathbb{P}\left[2 \mathcal{M}_{t}+2 \mathcal{R}_{t}>3 \mathcal{M}_{t}\right]=\mathbb{P}\left[\mathcal{R}_{t}>\frac{1}{2} \mathcal{M}_{t}\right]$. Now, from Lemma 4.3 equation (4.12), one gets $\mathbb{P}\left[\gamma_{Y_{t}}>3 \mathcal{M}_{t}\right] \leq \bar{c}(n, k) \exp \left(-\frac{\left|\xi_{1, n}\right|^{2}}{4 \bar{c}(n, k) t}\right)$. Therefore, for $\left|\xi_{1, n}\right| \geq K t^{1 / 2}$ and $K$ large enough, we get $\mathbb{E}\left[\Gamma_{Y_{t}}^{q}\right] \geq \frac{1}{2\left(3 \mathcal{M}_{t}\right)^{q}}$, which thanks to (4.10) completes the proof.

\section{Controls of the weight for the integration by parts.}

From Proposition 3.6 and Corollary 3.7, we derive

$$
\begin{aligned}
p_{Y_{t}}\left(\xi_{n+1}-x_{n+1}\right) & =\mathbb{E}\left[H_{t} \mathbb{I}_{Y_{t}>\xi_{n+1}-x_{n+1}}\right] \\
H_{t} & =-\left\langle D \Gamma_{Y_{t}}, D Y_{t}\right\rangle_{L^{2}(0, t)}+\Gamma_{Y_{t}} L Y_{t}=\gamma_{Y_{t}}^{-2}\left\langle D \gamma_{Y_{t}}, D Y_{t}\right\rangle_{L^{2}(0, t)}+\Gamma_{Y_{t}} L Y_{t} \\
& :=H_{t}^{1}+H_{t}^{2}
\end{aligned}
$$

using the chain rule for the last but one identity.

We have the following $L^{q}(\mathbb{P}), q \geq 1$, bounds for the random variable $H_{t}$.

Proposition 4.6 (Estimates for the Malliavin weight) Assume that $\left|x_{1, n}\right| \vee\left|\xi_{1, n}\right| \geq K t^{1 / 2}$ for $K$ large enough. Then, for all $q \in[1,+\infty)$ there exists $C_{q, 4.6}:=C_{q, 4.6}(n, k, K) \geq 1$ s.t.

$$
\left\|H_{t}\right\|_{q} \leq \frac{C_{q, 4.6}}{\left(\left|x_{1, n}\right|^{(k-1)}+\left|\xi_{1, n}\right|^{(k-1)}\right) t^{3 / 2}} .
$$


Proof: Control of $H_{t}^{1}$. From (4.19) we get for all given $q \geq 1$,

$$
\begin{array}{r}
\left\|H_{t}^{1}\right\|_{q}:=\mathbb{E}\left[\gamma_{Y_{t}}^{-2 q}\left|\left\langle D \gamma_{Y_{t}}, D Y_{t}\right\rangle_{L^{2}(0, t)}\right|^{q}\right]^{1 / q} \leq \mathbb{E}\left[\gamma_{Y_{t}}^{-4 q}\right]^{1 / 2 q} \mathbb{E}\left[\left|\left\langle D \gamma_{Y_{t}}, D Y_{t}\right\rangle_{L^{2}(0, t)}\right|^{2 q}\right]^{1 / 2 q} \\
\leq \frac{C_{q, 4.5}}{t^{6}\left(\left|\xi_{1, n}\right|^{2(k-1)}+\left|x_{1, n}\right|^{2(k-1)}\right)^{2}} \mathbb{E}\left[\left|\left\langle D \gamma_{Y_{t}}, D Y_{t}\right\rangle_{L^{2}(0, t)}\right|^{2 q}\right]^{1 / 2 q} \\
\leq \frac{C_{q, 4.5}}{t^{6}\left(\left|\xi_{1, n}\right|^{2(k-1)}+\left|x_{1, n}\right|^{2(k-1)}\right)^{2}} \mathbb{E}\left[\left|D \gamma_{Y_{t}}\right|_{L^{2}(0, t)}^{4 q}\right]^{1 / 4 q} \mathbb{E}\left[\left|D Y_{t}\right|_{L^{2}(0, t)}^{4 q}\right]^{1 / 4 q},
\end{array}
$$

using Lemma 4.5 for the last but one inequality. Now, from equations (4.6), (4.8), using the notations of Lemma 4.5,

$$
\begin{aligned}
\mathbb{E}\left[\left|D Y_{t}\right|_{L^{2}(0, t)}^{4 q}\right]^{1 / 4 q} & =\mathbb{E}\left[\left(\int_{0}^{t} d s\left|D_{s} Y_{t}\right|^{2}\right)^{2 q}\right]^{1 / 4 q}:=\mathbb{E}\left[\gamma_{Y_{t}}^{2 q}\right]^{1 / 4 q} \\
& \leq\left(2^{4 q-1}\left\{\mathcal{M}_{t}^{2 q}+\mathbb{E}\left[\mathcal{R}_{t}^{2 q}\right]\right\}\right)^{1 / 4 q} \leq 2^{1-1 / 4 q}\left\{\mathcal{M}_{t}^{1 / 2}+\mathbb{E}\left[\mathcal{R}_{t}^{2 q}\right]^{1 / 4 q}\right\} .
\end{aligned}
$$

On the one hand equation (4.10) in Lemma 4.3 readily gives $\mathcal{M}_{t}^{1 / 2} \leq C t^{3 / 2}\left(\left|x_{1, n}\right|^{k-1}+\right.$ $\left.\left|\xi_{1, n}\right|^{k-1}\right)$. On the other hand, equation (4.12) of the same Lemma yields

$$
\mathbb{E}\left[\left|\mathcal{R}_{t}\right|^{2 q}\right]^{1 / 4 q} \leq C(k, q)\left(t^{3 / 2}\left(\left|\xi_{1, n}\right|^{k-1} \vee\left|x_{1, n}\right|^{k-1}\right) K^{-1} k / 2\right) .
$$

Hence, there exists $C_{1}:=C_{1}(n, k, q, K)$ s.t.

$$
\mathbb{E}\left[\left|D Y_{t}\right|_{L^{2}(0, t)}^{4 q}\right]^{1 / 4 q}=\mathbb{E}\left[\gamma_{Y_{t}}^{2 q}\right]^{1 / 4 q} \leq C_{1} t^{3 / 2}\left(\left|\xi_{1, n}\right|^{k-1}+\left|x_{1, n}\right|^{k-1}\right) .
$$

In order to get a bound for $\left\|H_{t}^{1}\right\|_{q}$, it remains to control $\mathbb{E}\left[\left|D \gamma_{Y_{t}}\right|_{L^{2}(0, t)}^{4 q}\right]^{1 / 4 q}$. Equation (4.6) and the chain rule yield that for all $u_{2} \in[0, t], D_{u_{2}} \gamma_{Y_{t}}=2 \int_{0}^{t} d u_{1} D_{u_{2}} D_{u_{1}} Y_{t} \times D_{u_{1}} Y_{t}$. We get

$$
\begin{aligned}
\mathbb{E}\left[\left|D \gamma_{Y_{t}}\right|_{L^{2}(0, t)}^{4 q}\right]^{1 / 4 q} & \leq 4 \mathbb{E}\left[\gamma_{Y_{t}}^{4 q}\right]^{1 / 8 q} \mathbb{E}\left[\left(\int_{0}^{t} d u_{1} \int_{0}^{t} d u_{2}\left|D_{u_{2}, u_{1}} Y_{t}\right|^{2}\right)^{4 q}\right]^{1 / 8 q} \\
& \leq C_{2} t^{3 / 2}\left(\left|\xi_{1, n}\right|^{k-1}+\left|x_{1, n}\right|^{k-1}\right) \mathbb{E}\left[\left|D^{2} Y_{Y_{t}}\right|_{L^{2}\left((0, t)^{2}\right)}\right]^{1 / 8 q}
\end{aligned}
$$

$C_{2}:=C_{2}(n, k, q, K)$ using (4.21) for the last inequality.

With the notations of equations (4.6), (4.8) we set for all $u_{1} \in[0, t]$,

$$
D_{u_{1}} Y_{t}:=\sum_{i=1}^{k / 2} M_{i}\left(u_{1}, t, x_{1, n}, \xi_{1, n}\right):=\sum_{i=1}^{k / 2} \bar{M}_{i}\left(u_{1}, t\right),
$$

for simplicity.

Observe now that for all $i \in \llbracket 2, k / 2 \rrbracket, u_{2} \in[0, t]$,

$$
\begin{array}{r}
D_{u_{2}} \bar{M}_{i}\left(u_{1}, t\right)=C_{k / 2}^{i} \int_{u_{1} \vee u_{2}}^{t} d v\left|m\left(v, t, x_{1, n}, \xi_{1, n}\right)\right|^{k-2 i}\left\{\left|W_{v}^{0, t}\right|^{2}+2\left\langle m\left(v, t, x_{1, n}, \xi_{1, n}\right), W_{v}^{0, t}\right\rangle\right\}^{i-2} \\
\times 2 i \frac{(t-v)^{2}}{\left(t-u_{1}\right)\left(t-u_{2}\right)}\left\{2(i-1)\left(W_{v}^{0, t}+m\left(v, t, x_{1, n}, \xi_{1, n}\right)\right) \otimes\left(W_{v}^{0, t}+m\left(v, t, x_{1, n}, \xi_{1, n}\right)\right)\right. \\
\left.+\left\{\left|W_{v}^{0, t}\right|^{2}+2\left\langle m\left(v, t, x_{1, n}, \xi_{1, n}\right), W_{v}^{0, t}\right\rangle\right\} I_{n}\right\}, \\
D_{u_{2}} \bar{M}_{1}\left(u_{1}, t\right)=k \int_{u_{1} \vee u_{2}}^{t} d v\left|m\left(v, t, x_{1, n}, \xi_{1, n}\right)\right|^{k-2} \frac{(t-v)^{2}}{\left(t-u_{1}\right)\left(t-u_{2}\right)} I_{n} .
\end{array}
$$


From the above equations, assuming once again w.l.o.g. $\left|\xi_{1, n}\right| \geq\left|x_{1, n}\right|$, the arguments used in Lemma 4.3 yield:

$$
\begin{aligned}
\mathbb{E}\left[\left|D^{2} Y_{t}\right|_{L^{2}\left((0, t)^{2}\right)}^{8 q}\right]^{1 / 8 q} \leq C \mathbb{E}\left[\left.\left|\int_{[0, t]^{2}} d u_{1} d u_{2}\left(t-u_{1} \vee u_{2}\right)^{2}\right| \xi_{1, n}\right|^{2(k-2)}\right. \\
\left.\times\left.\left(1+\sum_{i=2}^{k / 2}\left|\xi_{1, n}\right|^{4(1-i)} \sup _{u \in[0, t]}\left|W_{u}^{0, t}\right|^{4(i-1)}\right)\right|^{4 q}\right]^{1 / 8 q} \\
\leq C t^{2}\left|\xi_{1, n}\right|^{k-2}\left(1+\sum_{i=2}^{k / 2}\left|\xi_{1, n}\right|^{2(1-i)} \mathbb{E}\left[\sup _{u \in[0, t]}\left|W_{u}^{0, t}\right|^{16 q(i-1)}\right]^{1 / 8 q}\right) \\
\quad \stackrel{\text { Prop. } 4.2}{ } \leq t^{2}\left|\xi_{1, n}\right|^{k-2}\left(1+\sum_{i=2}^{k / 2}\left(\frac{t^{1 / 2}}{\left|\xi_{1, n}\right|}\right)^{2(i-1)}\right),
\end{aligned}
$$

where $C:=C(n, k, q)$ may change from line to line. Recalling that $\left|\xi_{1, n}\right| \vee\left|x_{1, n}\right| \geq K t^{1 / 2}$ we obtain

$$
\mathbb{E}\left[\left|D^{2} Y_{t}\right|_{L^{2}\left((0, t)^{2}\right)}^{8 q}\right]^{1 / 8 q} \leq C t^{2}\left|\xi_{1, n}\right|^{k-2}, C:=C(n, k, q, K) .
$$

Plugging the above equation into (4.22) we derive that

$$
\mathbb{E}\left[\left|D \gamma_{Y_{t}}^{4 q}\right|_{L^{2}(0, t)}\right]^{1 / 4 q} \leq C t^{7 / 2}\left|\xi_{1, n}\right|^{2 k-3},
$$

which together with (4.21) and (4.20), eventually yields

$$
\left\|H_{t}^{1}\right\|_{q} \leq \frac{\bar{C}_{1}}{t\left|\xi_{1, n}\right|^{k}} \leq \frac{C K^{-1}}{t^{3 / 2}\left|\xi_{1, n}\right|^{k-1}}, \bar{C}_{1}:=\bar{C}_{1}(n, k, q, K) .
$$

Control of $H_{t}^{2}$. From (4.19) and Lemma 4.5, for all $q \geq 1$, we get

$$
\left\|H_{t}^{2}\right\|_{q} \leq \mathbb{E}\left[\left|\Gamma_{Y_{t}}\right|^{2 q}\right]^{1 / 2 q} \mathbb{E}\left[\left|L Y_{t}\right|^{2 q}\right]^{1 / 2 q} \leq \frac{C_{q}}{t^{3}\left(\left|x_{1, n}\right|^{2(k-1)}+\left|\xi_{1, n}\right|^{2(k-1)}\right)} \mathbb{E}\left[\left|L Y_{t}\right|^{2 q}\right]^{1 / 2 q} .
$$

Now, since $L Y_{t}=\delta\left(D Y_{t}\right)$, the idea is to provide a chaotic representation of $D Y_{t}$. To do that, we use Proposition 3.4 (Stroock's formula see [38]). For a given $u_{1} \in[0, t]$, recalling $D_{u_{1}} Y_{t}:=\sum_{i=1}^{k / 2} \bar{M}_{i}\left(u_{1}, t\right)$ where $\bar{M}_{i}\left(u_{1}, t\right) \in \mathbb{R}^{n}$ is a random contribution involving Wiener chaos up to order $2 i-1$, one has:

$$
\begin{aligned}
\bar{M}_{i}\left(u_{1}, t\right) & =\mathbb{E}\left[\bar{M}_{i}\left(u_{1}, t\right)\right]+\sum_{l=1}^{2 i-1} I_{l}\left(g_{l}^{i}\left(., u_{1}, t\right)\right), \\
I_{l}\left(g_{l}^{i}\left(., u_{1}, t\right)\right) & :=\int_{0}^{t} \int_{0}^{v_{1}} \cdots \int_{0}^{v_{l-1}} g_{l}^{i}\left(v_{1}, \cdots, v_{l}, u_{1}, t\right) \otimes d W_{v_{l}} \otimes \cdots \otimes d W_{v_{1}}, \\
g_{l}^{i}\left(v_{1}, \cdots, v_{l}, u_{1}, t\right) & :=\mathbb{E}\left[D_{v_{l}, \cdots, v_{1}} \bar{M}_{i}\left(u_{1}, t\right)\right] \in\left(\mathbb{R}^{n}\right)^{\otimes(l+1)},\left(d W_{v_{l}} \otimes \cdots \otimes d W_{v_{1}}\right) \in\left(\left(\mathbb{R}^{n}\right)^{\otimes l}\right)^{*} .
\end{aligned}
$$


Hence, $D_{u_{1}} Y_{t}:=g_{0}\left(u_{1}, t\right)+\sum_{l=1}^{k-1} I_{l}\left(g_{l}\left(., u_{1}, t\right)\right)$, where $g_{0}\left(u_{1}, t\right):=\sum_{i=1}^{k / 2} \mathbb{E}\left[\bar{M}_{i}\left(u_{1}, t\right)\right]$ and for all $l \in \llbracket 1, k-1 \rrbracket, \quad g_{l}\left(v_{1}, \cdots, v_{l}, u_{1}, t\right):=\sum_{i=\lfloor l / 2\rfloor+1}^{k / 2} g_{l}^{i}\left(v_{1}, \cdots, v_{l}, u_{1}, t\right)$, so that

$$
L Y_{t}=\int_{0}^{t} g_{0}\left(u_{1}, t\right) \otimes d W_{u_{1}}+\sum_{l=2}^{k} I_{l}\left(g_{l-1}(., t)\right):=\sum_{l=1}^{k} I_{l}\left(g_{l-1}(., t)\right)
$$

Similarly to the proof performed to control $\mathbb{E}\left[\left|\mathcal{R}_{t}\right|^{2 q}\right]^{1 / 4 q}$ of (4.12) in Lemma 4.3, we obtain that there exists $C:=C(n, k)$ s.t. for all $l \in \llbracket 0, k-1 \rrbracket$ and for all $\left(v_{1}, \cdots, v_{l}, u_{1}\right) \in[0, t]^{l+1}$ :

$$
\left|g_{l}\left(v_{1}, \cdots, v_{l}, u_{1}, t\right)\right| \leq C t\left(\left|\xi_{1, n}\right|^{k-(l+1)}+\left|x_{1, n}\right|^{k-(l+1)}\right) .
$$

Therefore,

$$
\begin{aligned}
\mathbb{E}\left[\left|L Y_{t}\right|^{2 q}\right]^{1 / 2 q} & \leq C \sum_{l=1}^{k} t^{1+l / 2}\left(\left|\xi_{1, n}\right|^{k-l}+\left|x_{1, n}\right|^{k-l}\right) \\
& \leq C t^{3 / 2}\left(\left|\xi_{1, n}\right|^{k-1}+\left|x_{1, n}\right|^{k-1}\right)\left\{\sum_{l=1}^{k} t^{(l-1) / 2}\left(\left|\xi_{1, n}\right|^{1-l}+\left|x_{1, n}\right|^{1-l}\right)\right\}
\end{aligned}
$$

where $C:=C(n, k, q)$ may change from line to line. Recalling that $\left|\xi_{1, n}\right| \vee\left|x_{1, n}\right| \geq K t^{1 / 2}$, we derive from (4.24) that there exists $\bar{C}_{2}:=\bar{C}_{2}(n, k, q, K)$ s.t.

$$
\left\|H_{t}^{2}\right\|_{q} \leq \frac{\bar{C}_{2}}{t^{3 / 2}\left(\left|\xi_{1, n}\right|^{k-1}+\left|x_{1, n}\right|^{k-1}\right)}
$$

which together with (4.23) and (4.19) completes the proof.

\subsubsection{Non Gaussian regime}

We now consider the case $\left|x_{1, n}\right| \vee\left|\xi_{1, n}\right| \leq K t^{1 / 2}$, which corresponds to a diagonal regime of the non-degenerate component w.r.t. the characteristic time scale. It turns out that the characteristic time-scale of the density $p_{Y_{t}}\left(\xi_{n+1}-x_{n+1}\right)$ is $t^{1+k / 2}$. Indeed, we have the following result.

Proposition 4.7 (Estimates for the Malliavin weight in Non Gaussian regime ) Let $K>0$ be given and assume that $\left|x_{1, n}\right| \vee\left|\xi_{1, n}\right| \leq K t^{1 / 2}$. For every $q \geq 1$ there exists $C_{q, 4.7}:=C_{q, 4.7}(n, k, K)$ s.t.

$$
\left\|H_{t}\right\|_{q} \leq \frac{C_{q, 4.7}}{t^{1+k / 2}} .
$$


Proof. For $t>0$ write:

$$
\begin{aligned}
Y_{t}=\int_{0}^{t}\left|x_{1, n} \frac{t-u}{t}+\xi_{1, n} \frac{u}{t}+W_{u}^{0, t}\right|^{k} d u & =t^{1+k / 2} \int_{0}^{1}\left|\frac{x_{1, n}}{t^{1 / 2}}(1-u)+\frac{\xi_{1, n}}{t^{1 / 2}} u+\frac{W_{u t}^{0, t}}{t^{1 / 2}}\right|^{k} d u \\
& =: t^{1+k / 2} \bar{Y}_{1}^{t} .
\end{aligned}
$$

Thus:

$$
\begin{aligned}
p_{Y_{t}}\left(\xi_{n+1}-x_{n+1}\right): & :=-\partial_{\xi_{n+1}} \mathbb{P}\left[Y_{t}>\xi_{n+1}-x_{n+1}\right]=-\partial_{\xi_{n+1}} \mathbb{P}\left[\bar{Y}_{1}^{t}>\frac{\xi_{n+1}-x_{n+1}}{t^{1+k / 2}}\right] \\
& =\frac{1}{t^{1+k / 2}} p_{\bar{Y}_{1}^{t}}\left(\frac{\xi_{n+1}-x_{n+1}}{t^{1+k / 2}}\right) .
\end{aligned}
$$

From Corollary 3.7 (Malliavin representation of the densities), we obtain:

$$
\begin{aligned}
p_{Y_{t}}\left(\xi_{n+1}-x_{n+1}\right) & =\mathbb{E}\left[H\left(Y_{t}, 1\right) \mathbb{I}_{Y_{t}>\xi_{n+1}-x_{n+1}}\right]=\frac{1}{t^{1+k / 2}} \mathbb{E}\left[H\left(\bar{Y}_{1}^{t}, 1\right) \mathbb{I}_{\bar{Y}_{1}^{t}>\frac{\xi_{n+1}-x_{n+1}}{t^{1+k / 2}}}\right] \\
& =\frac{1}{t^{1+k / 2}} \mathbb{E}\left[H\left(\bar{Y}_{1}^{t}, 1\right) \mathbb{I}_{Y_{t}>\xi_{n+1}-x_{n+1}}\right],
\end{aligned}
$$

so that $H_{t}:=H\left(Y_{t}, 1\right)=t^{-(1+k / 2)} H\left(\bar{Y}_{1}^{t}, 1\right):=t^{-(1+k / 2)} H_{1}^{t}$. Hence, for all $q \geq 1$,

$$
\left\|H_{t}\right\|_{q} \leq \frac{1}{t^{1+k / 2}}\left\|H_{1}^{\bar{Y}_{1}^{t}}\right\|_{q}
$$

Now, as a consequence of the Brownian scaling we get $\left(\frac{W_{u t}^{0, t}}{t^{1 / 2}}\right)_{u \in[0,1]} \stackrel{(\text { law })}{=}\left(W_{u}^{0,1}\right)_{u \in[0,1]}$ so that $\bar{Y}_{1}^{t} \stackrel{\text { (law) }}{=} t^{1+k / 2} \int_{0}^{1}\left|\frac{x_{1, n}}{t^{1 / 2}}(1-u)+\frac{\xi_{1, n}}{t^{1 / 2}} u+W_{u}^{0,1}\right|^{k} d u$. Recalling that $\left|\frac{x_{1, n}}{t^{1 / 2}}\right| \vee\left|\frac{\xi_{1, n}}{t^{1 / 2}}\right| \leq K$ we derive that the usual techniques used to prove the non degeneracy of the Malliavin covariance matrix under Hörmander's condition (see e.g. Norris [31] or Nualart [32]) yield that there exists $C_{q}:=C_{q}(n, k, K) \in \mathbb{R}^{+*}$ s.t. $\left\|H_{1}^{\bar{Y}_{1}^{t}}\right\|_{q} \leq C_{q}$ which from (4.27) concludes the proof. The crucial tool here is the global scaling.

\subsection{Deviation estimates}

\subsubsection{Off-diagonal bounds}

From the Malliavin representation of the density given by (4.19), to derive off-diagonal bounds on the density, it remains to give estimates on $\mathbb{P}\left[Y_{t}>\xi_{n+1}-x_{n+1}\right]$.

Lemma 4.8 (Off-diagonal bounds) Let $U_{t}^{k}(x, \xi):=\xi_{n+1}-x_{n+1}-\frac{2^{k-1}}{k+1}\left(\left|x_{1, n}\right|^{k}+\left|\xi_{1, n}\right|^{k}\right) t$, and assume that $U_{t}^{k}(x, \xi)>0$. Then, there exists $C_{4.8}:=C_{4.8}(n, k)$ s.t.

(i) If $\left|x_{1, n}\right| \vee\left|\xi_{1, n}\right| \geq K t^{1 / 2}$ for a given $K>0$,

$$
\begin{array}{r}
\mathbb{P}\left[Y_{t}>\xi_{n+1}-x_{n+1}\right] \leq C_{4.8}\left\{\exp \left(-C_{4.8}^{-1} \frac{U_{t}^{k}(x, \xi)^{2}}{\left(\left|x_{1, n}\right|^{k-1}+\left|\xi_{1, n}\right|^{k-1}\right)^{2} t^{3}}\right)\right. \\
\left.+\exp \left(-C_{4.8}^{-1} \frac{\left|x_{1, n}\right|^{2}+\left|\xi_{1, n}\right|^{2}}{t}\right) \sum_{i=1}^{k / 2} \exp \left(-C_{4.8}^{-1} \frac{U_{t}^{k}(x, \xi)^{1 / i}}{\left\{\left|x_{1, n}\right|^{k-2 i}+\left|\xi_{1, n}\right|^{k-2 i}\right\}^{1 / i} t^{1+1 / i}}\right)\right\} .
\end{array}
$$


(ii) If $\left|x_{1, n}\right| \vee\left|\xi_{1, n}\right| \leq K t^{1 / 2}$ for the same previous $K$,

$$
\mathbb{P}\left[Y_{t}>\xi_{n+1}-x_{n+1}\right] \leq C_{4.8}\left\{\exp \left(-C_{4.8}^{-1} \frac{U_{t}^{k}(x, \xi)^{2}}{t^{k+2}}\right)+\sum_{i=1}^{k / 2} \exp \left(-C_{4.8}^{-1} \frac{U_{t}^{k}(x, \xi)^{1 / i}}{t^{k /(2 i)+1 / i}}\right)\right\} .
$$

Proof. We only prove point (i), the second point can be derived in a similar way. According with (4.5), we first decompose $Y_{t}$ as

$$
Y_{t}=\int_{0}^{t}\left|m\left(u, t, x_{1, n}, \xi_{1, n}\right)\right|^{k} d u+M_{t}^{k}\left(x_{1, n}, \xi_{1, n}\right)+R_{t}^{k}\left(x_{1, n}, \xi_{1, n}\right)
$$

where

$$
\begin{gathered}
M_{t}^{k}\left(x_{1, n}, \xi_{1, n}\right):=k \int_{0}^{t}\left|m\left(u, t, x_{1, n}, \xi_{1, n}\right)\right|^{k-2}\left\langle m\left(u, t, x_{1, n}, \xi_{1, n}\right), W_{u}^{0, t}\right\rangle d u, \\
R_{t}^{k}\left(x_{1, n}, \xi_{1, n}\right):=\frac{k}{2} \int_{0}^{t}\left|m\left(u, t, x_{1, n}, \xi_{1, n}\right)\right|^{k-2}\left|W_{u}^{0, t}\right|^{2} d u \\
+\sum_{i=2}^{k / 2} C_{k / 2}^{i} \int_{0}^{t}\left|m\left(u, t, x_{1, n}, \xi_{1, n}\right)\right|^{k-2 i}\left(2\left\langle m\left(u, t, x_{1, n}, \xi_{1, n}\right), W_{u}^{0, t}\right\rangle+\left|W_{u}^{0, t}\right|^{2}\right)^{i} d u
\end{gathered}
$$

then we have

$$
\begin{gathered}
\mathbb{P}\left[Y_{t}>\xi_{n+1}-x_{n+1}\right]=\mathbb{P}\left[M_{t}^{k}\left(x_{1, n}, \xi_{1, n}\right)+R_{t}^{k}\left(x_{1, n}, \xi_{1, n}\right)>\right. \\
\left.\xi_{n+1}-x_{n+1}-\int_{0}^{t}\left|m\left(u, t, x_{1, n}, \xi_{1, n}\right)\right|^{k} d u\right] .
\end{gathered}
$$

Note that all the terms in $R_{t}^{k}\left(x_{1, n}, \xi_{1, n}\right)$ have characteristic time scales that are in small time negligible with respect to the one of the Gaussian contribution $M_{t}^{k}\left(x_{1, n}, \xi_{1, n}\right)$. Moreover

$$
M_{t}^{k}\left(x_{1, n}, \xi_{1, n}\right) \leq 2^{k-2}\left(\left|x_{1, n}\right|^{k-1}+\left|\xi_{1, n}\right|^{k-1}\right) t \sup _{u \in[0, t]}\left|W_{u}^{0, t}\right|=: \widetilde{M}_{t}^{k}\left(x_{1, n}, \xi_{1, n}\right) .
$$

Since by assumption $U_{t}^{k}(x, \xi)<\xi_{n+1}-x_{n+1}-\int_{0}^{t}\left|m\left(u, t, x_{1, n}, \xi_{1, n}\right)\right|^{k} d u$, one gets:

$$
\begin{aligned}
\mathbb{P}\left[Y_{t}>\xi_{n+1}-x_{n+1}\right] \leq & \mathbb{P}\left[\left(\widetilde{M}_{t}^{k}+R_{t}^{k}\right)\left(x_{1, n}, \xi_{1, n}\right)>U_{t}^{k}(x, \xi)\right] \leq \\
& \mathbb{P}\left[2 \widetilde{M}_{t}^{k}\left(x_{1, n}, \xi_{1, n}\right)>U_{t}^{k}(x, \xi)\right] \\
+ & \mathbb{P}\left[\left(\widetilde{M}_{t}^{k}+R_{t}^{k}\right)\left(x_{1, n}, \xi_{1, n}\right)>U_{t}^{k}(x, \xi)\right]^{1 / 2} \\
& \times \mathbb{P}\left[R_{t}^{k}\left(x_{1, n}, \xi_{1, n}\right) \geq \widetilde{M}_{t}^{k}\left(x_{1, n}, \xi_{1, n}\right)\right]^{1 / 2} .
\end{aligned}
$$

Standard computations, similar to the ones performed to prove the deviation estimate in (4.12) in Lemma 4.3, give that there exist $C_{1}:=C_{1}(k), C_{2}:=C_{2}(n, k) \geq 1$ s.t.

$$
\begin{aligned}
\mathbb{P}\left[R_{t}^{k}\left(x_{1, n}, \xi_{1, n}\right) \geq \widetilde{M}_{t}^{k}\left(x_{1, n}, \xi_{1, n}\right)\right] & \leq(k-1) \mathbb{P}\left[\sup _{u \in[0, t]}\left|W_{u}^{0, t}\right| \geq C_{1}\left\{\left|x_{1, n}\right|+\left|\xi_{1, n}\right|\right\}\right] \\
& \leq C_{2} \exp \left(-C_{2}^{-1} \frac{\left|x_{1, n}\right|^{2}+\left|\xi_{1, n}\right|^{2}}{t}\right) \\
\mathbb{P}\left[\widetilde{M}_{t}^{k}\left(x_{1, n}, \xi_{1, n}\right)>U_{t}^{k}(x, \xi) / 2\right] & \leq C_{2} \exp \left(-C_{2}^{-1} \frac{U_{t}^{k}(x, \xi)^{2}}{\left(\left|x_{1, n}\right|^{k-1}+\left|\xi_{1, n}\right|^{k-1}\right) t^{3}}\right) .
\end{aligned}
$$


On the other hand, we have:

$$
\begin{array}{r}
\mathbb{P}\left[\left(\widetilde{M}_{t}^{k}+R_{t}^{k}\right)\left(x_{1, n}, \xi_{1, n}\right) \geq U_{t}^{k}(x, \xi)\right] \\
\leq \mathbb{P}\left[\widetilde{M}_{t}^{k}\left(x_{1, n}, \xi_{1, n}\right) \geq \frac{1}{2} U_{t}^{k}(x, \xi)\right]+\mathbb{P}\left[R_{t}^{k}\left(x_{1, n}, \xi_{1, n}\right) \geq \frac{1}{2} U_{t}^{k}(x, \xi)\right] .
\end{array}
$$

Now,

$$
\begin{aligned}
& \mathbb{P}\left[R_{t}^{k}\left(x_{1, n}, \xi_{1, n}\right) \geq \frac{1}{2} U_{t}^{k}(x, \xi)\right] \\
& \leq \mathbb{P}\left[\frac{2^{(k-3) \vee 0} k}{2(k-1)}\left\{\left|x_{1, n}\right|^{k-2}+\left|\xi_{1, n}\right|^{k-2}\right\} t \sup _{u \in[0, t]}\left|W_{u}^{0, t}\right|^{2} \geq \frac{1}{2(k-1)} U_{t}^{k}(x, \xi)\right] \\
& +\sum_{i=2}^{k / 2}\left\{\mathbb{P}\left[C_{k / 2}^{i} \frac{2^{k-i-2}}{k-2 i+1}\left\{\left|x_{1, n}\right|^{k-2 i}+\left|\xi_{1, n}\right|^{k-2 i}\right\} t \sup _{u \in[0, t]}\left|W_{u}^{0, t}\right|^{2 i} \geq \frac{1}{2(k-1)} U_{t}^{k}(x, \xi)\right]\right. \\
& \left.+\mathbb{P}\left[C_{k / 2}^{i} \frac{2^{k+i-2}}{k-i+1}\left\{\left|x_{1, n}\right|^{k-i}+\left|\xi_{1, n}\right|^{k-i}\right\} t \sup _{u \in[0, t]}\left|W_{u}^{0, t}\right|^{i} \geq \frac{1}{2(k-1)} U_{t}^{k}(x, \xi)\right]\right\} \\
& :=P_{1}+\sum_{i=2}^{k / 2}\left(P_{2}^{i}+P_{3}^{i}\right) \text {. }
\end{aligned}
$$

From Proposition 4.2 one gets that there exists $C_{3}:=C_{3}(k, n) \geq 1$ s.t.

$$
\begin{aligned}
& P_{1} \leq C_{3} \exp \left(-C_{3}^{-1} \frac{U_{t}^{k}(x, \xi)}{\left\{\left|x_{1, n}\right|^{k-2}+\left|\xi_{1, n}\right|^{k-2}\right\} t^{2}}\right), \forall i \in \llbracket 2, k / 2 \rrbracket, \\
& P_{2}^{i} \leq C_{3} \exp \left(-C_{3}^{-1} \frac{U_{t}^{k}(x, \xi)^{1 / i}}{\left\{\left|x_{1, n}\right|^{k-2 i}+\left|\xi_{1, n}\right|^{k-2 i}\right\}^{1 / i} t^{1+1 / i}}\right), \\
& P_{3}^{i} \leq C_{3} \exp \left(-C_{3}^{-1} \frac{U_{t}^{k}(x, \xi)^{2 / i}}{\left\{\left|x_{1, n}\right|^{k-i}+\left|\xi_{1, n}\right|^{k-i}\right\}^{2 / i} t^{1+2 / i}}\right) .
\end{aligned}
$$

Hence, plugging (4.32) in (4.31) we derive the claim from (4.31), (4.30), (4.29) and (4.28).

\subsubsection{Auxiliary deviation estimates}

Still from the Malliavin representation of the density given by (4.19), when $\xi_{n+1}-x_{n+1}$ is small, that is when for the degenerate component the starting and final points are close, we have to give estimates on $\mathbb{P}\left[Y_{t} \leq \xi_{n+1}-x_{n+1}\right]$ (small and moderate deviations).

Proposition 4.9 There exist constants $\left(c_{1}, c_{2}\right):=\left(c_{1}, c_{2}\right)(n, k)$ s.t. for all $\left(x_{1, n}, \xi_{1, n}\right) \in$ $\left(\mathbb{R}^{n} \backslash\{0\}\right)^{2}, \xi_{n+1}>x_{n+1}$ and $t \geq 2^{k+3} \frac{\xi_{n+1}-x_{n+1}}{\left|x_{1, n}\right|^{k}+\left|\xi_{1, n}\right|^{k}}:$

$$
\mathbb{P}\left[Y_{t} \leq \xi_{n+1}-x_{n+1}\right] \leq c_{1} \exp \left(-c_{2} \frac{\left|x_{1, n}\right|^{2+k}+\left|\xi_{1, n}\right|^{2+k}}{\xi_{n+1}-x_{n+1}}\right)
$$


For a given $K \geq 0$, if $t \geq\left[\left(\xi_{n+1}-x_{n+1}\right) \frac{3}{4}(64 K)^{k}\right]^{2 /(k+2)}$, and $\left|x_{1, n}\right| \vee\left|\xi_{1, n}\right| \leq K t^{1 / 2}$, then there exist $\left(\bar{c}_{1}, \bar{c}_{2}\right):=\left(\bar{c}_{1}, \bar{c}_{2}\right)(n, k, K)$ :

$$
\mathbb{P}\left[Y_{t} \leq \xi_{n+1}-x_{n+1}\right] \leq \bar{c}_{1} \exp \left(-\bar{c}_{2} \frac{t^{1+2 / k}}{\left(\xi_{n+1}-x_{n+1}\right)^{2 / k}}\right) .
$$

Proof. We first begin with the proof of (4.33). As in the previous sections, we can assume w.l.o.g. that $\left|x_{1, n}\right| \geq\left|\xi_{1, n}\right|$.For $s \in[0, t]$, we define $\widetilde{X}_{s}:=x_{1, n} \frac{t-s}{t}+\xi_{1, n} \frac{s}{t}+W_{s}^{0, t}$ (where $\left(W_{s}^{0, t}\right)_{s \in[0, t]}$ is a standard $n$-dimensional Brownian Bridge on $\left.[0, t]\right)$, so that $Y_{t}=\int_{0}^{t}\left|\widetilde{X}_{s}\right|^{k} d s$. Let us also set $\tau_{\left|x_{1, n}\right| / 2}:=\inf \left\{s \geq 0:\left|\widetilde{X}_{s}\right| \leq\left|x_{1, n}\right| / 2\right\}$. Consider now the event $A:=$ $\left\{\tau_{\left|x_{1, n}\right| / 2} \leq 2^{k} \frac{\xi_{n+1}-x_{n+1}}{\left|x_{1, n}\right|^{k}}\right\}$ and denote by $A^{C}$ its complementary. Observe that $\mathbb{P}\left[\int_{0}^{t}\left|\tilde{X}_{s}\right|^{k} d s \leq\right.$ $\left.\xi_{n+1}-x_{n+1}, A^{C}\right]=\mathbb{P}\left[\int_{0}^{2^{k} \frac{\xi_{n+1}-x_{n+1}}{\left|x_{1, n}\right|^{k}}}\left(\frac{\left|x_{1, n}\right|}{2}\right)^{k} d s<\int_{0}^{t}\left|\tilde{X}_{s}\right|^{k} d s \leq \xi_{n+1}-x_{n+1}, A^{C}\right]=0$. Thus, $\mathbb{P}\left[Y_{t} \leq \xi_{n+1}-x_{n+1}\right]=\mathbb{P}\left[Y_{t} \leq \xi_{n+1}-x_{n+1}, A\right] \leq \mathbb{P}[A]$. Now

$$
\begin{aligned}
& \mathbb{P}[A] \leq \mathbb{P}\left[\inf _{s \in\left[0,2^{k} \frac{\xi_{n+1}-x_{n+1}}{\left|x_{1, n}\right|^{k}}\right]}\left|\widetilde{X}_{s}\right| \leq\left|x_{1, n}\right| / 2\right]
\end{aligned}
$$

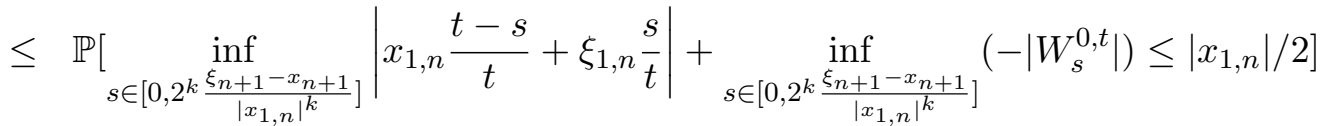

$$
\begin{aligned}
& \leq \mathbb{P}\left[\left|x_{1, n}\right| / 2+\inf _{s \in\left[0,2^{k} \frac{\xi_{n+1}-x_{n+1}}{\left|x_{1, n}\right|^{k}}\right]}\left(-\frac{s}{t}\right)\left\{\left|x_{1, n}\right|+\left|\xi_{1, n}\right|\right\}-\sup _{s \in\left[0,2^{k} \frac{\xi_{n+1}-x_{n+1}}{\left|x_{1, n}\right|^{k}}\right]}\left|W_{s}^{0, t}\right| \leq 0\right] \\
& \leq \mathbb{P}\left[\left|x_{1, n}\right|\left(1 / 2-\frac{2^{k+1}\left(\xi_{n+1}-x_{n+1}\right)}{\left|x_{1, n}\right|^{k} t}\right) \leq \sup _{s \in\left[0,2^{k} \frac{\xi_{n+1}-x_{n+1}}{\left|x_{1, n}\right|^{k}}\right]}\left|W_{s}^{0, t}\right|\right] \\
& \leq \mathbb{P}\left[\left|x_{1, n}\right| / 4 \leq \sup _{s \in\left[0,2^{k} \frac{\xi_{n+1}-x_{n+1}}{\left|x_{1, n}\right|^{k}}\right]}\left|W_{s}^{0, t}\right|\right]
\end{aligned}
$$

recalling $\left|x_{1, n}\right| \geq\left|\xi_{1, n}\right|$ and $t \geq 2^{k+3} \frac{\xi_{n+1}-x_{n+1}}{\left|x_{1, n}\right|^{k}}$ for the last two inequalities. From Proposition 4.2 we obtain:

$$
\mathbb{P}\left[Y_{t} \leq \xi_{n+1}-x_{n+1}\right] \leq \mathbb{P}[A] \leq c_{1} \exp \left(-c_{2} \frac{\left|x_{1, n}\right|^{2+k}}{\xi_{n+1}-x_{n+1}}\right),
$$

which from the assumption $\left|x_{1, n}\right| \geq\left|\xi_{1, n}\right|$ gives (4.33) up to a modification of $c_{2}$.

Let us now turn to (4.34). Introduce $I_{\beta}(t):=\int_{0}^{t} \mathbb{I}_{\left|\widetilde{X}_{s}\right|^{k} \leq \beta\left(\xi_{n+1}-x_{n+1}\right)} d s$ for a parameter $\beta>0$ to be fixed later on. Define the set $A_{\beta}:=\left\{I_{\beta}(t) \geq t / 4\right\}$. Observe that

$$
\begin{array}{r}
\mathbb{P}\left[\int_{0}^{t}\left|\widetilde{X}_{s}\right|^{k} d s \leq \xi_{n+1}-x_{n+1}, A_{\beta}^{C}\right]= \\
\mathbb{P}\left[\int_{0}^{t} \mathbb{I}_{\left|\widetilde{X}_{s}\right|^{k}>\beta\left(\xi_{n+1}-x_{n+1}\right)}\left|\widetilde{X}_{s}\right|^{k} d s \leq \int_{0}^{t}\left|\widetilde{X}_{s}\right|^{k} d s \leq \xi_{n+1}-x_{n+1}, A_{\beta}^{C}\right] \\
\leq \mathbb{P}\left[\beta\left(\xi_{n+1}-x_{n+1}\right) 3 t / 4<\int_{0}^{t}\left|\widetilde{X}_{s}\right|^{k} d s \leq \xi_{n+1}-x_{n+1}, A_{\beta}^{C}\right] .
\end{array}
$$


Choosing $\beta=\frac{4}{3 t}$ we get from the above inequality $\mathbb{P}\left[\int_{0}^{t}\left|\widetilde{X}_{s}\right|^{k} d s \leq \xi_{n+1}-x_{n+1}, A_{\beta}^{C}\right]=0$. Hence,

$$
\begin{aligned}
& \mathbb{P}\left[\int_{0}^{t}\left|\widetilde{X}_{s}\right|^{k} d s \leq \xi_{n+1}-x_{n+1}\right]=\mathbb{P}\left[\int_{0}^{t}\left|\widetilde{X}_{s}\right|^{k} d s \leq \xi_{n+1}-x_{n+1}, A_{\frac{4}{3 t}}\right] \leq \mathbb{P}\left[A_{\frac{4}{3 t}}\right] \\
& \leq \mathbb{P}\left[\int_{0}^{t} \mathbb{I}_{\left|\widetilde{X}_{s}\right|^{k} \leq \frac{3\left(\xi_{n+1}-x_{n+1}\right)}{4 t}} d s>t / 4\right] \leq \mathbb{P}\left[\int_{0}^{t} \mathbb{I}_{\left|\widetilde{X}_{s}^{1}\right|^{k} \leq \frac{3\left(\xi_{n+1}-x_{n+1}\right)}{4 t}} d s>t / 4\right] \\
& \leq \mathbb{P}\left[\int_{0}^{t} \mathbb{I}_{\left|x_{1} \frac{t-s}{t}+\xi_{1} \frac{s}{t}+B_{s}^{0, t}\right| \leq c(x, \xi, t, k)} d s>t / 4\right], \quad c(x, \xi, t, k):=\left(\frac{3\left(\xi_{n+1}-x_{n+1}\right)}{4 t}\right)^{1 / k}, \\
& \leq \mathbb{P}\left[\int_{0}^{t / 2} \mathbb{I}_{\left|x_{1} \frac{t-s}{t}+\xi_{1} \frac{s}{t}+B_{s}^{0, t}\right| \leq c(x, \xi, t, k)} d s>t / 8\right]+\mathbb{P}\left[\int_{t / 2}^{t} \mathbb{I}_{\left|x_{1} \frac{t-s}{t}+\xi_{1} \frac{s}{t}+B_{s}^{0, t}\right| \leq c(x, \xi, t, k)} d s>t / 8\right] \\
& :=P_{1}+P_{2},
\end{aligned}
$$

where $\left(B_{s}^{0, t}\right)_{s \in[0, t]}$ stands for a one-dimensional Brownian bridge on $[0, t]$. Observing that $\left(\bar{B}_{s}^{0, t}\right):=\left(B_{t-s}^{0, t}\right)_{s \in[0, t]}$ is also a Brownian bridge, we get that

$$
\begin{aligned}
P_{2} & :=\mathbb{P}\left[\int_{0}^{t / 2} d s \mathbb{I}_{\left|x_{1} \frac{s}{t}+\xi_{1} \frac{t-s}{t}+\bar{B}_{s}^{0, t}\right| \leq c(x, \xi, t, k)} d s>t / 8\right] \\
& =\mathbb{P}\left[\int_{0}^{t / 2} d s \mathbb{I}_{\left|x_{1} \frac{s}{t}+\xi_{1} \frac{t-s}{t}+B_{s}^{0, t}\right| \leq c(x, \xi, t, k)} d s>t / 8\right] .
\end{aligned}
$$

Since we assumed $\left|x_{1}\right| \vee\left|\xi_{1}\right| \leq K t^{1 / 2},\left|x_{1}\right|$ and $\left|\xi_{1}\right|$ have at most the same magnitude so that $P_{1}$ and $P_{2}$ can be handled exactly in the same way. Let us deal with $P_{1}$. The occupation time formula for semimartingales (see Chapter 6 in [34]) yields

$$
\int_{0}^{t / 2} \mathbb{I}_{\left|x_{1} \frac{t-s}{t}+\xi_{1} \frac{s}{t}+B_{s}^{0, t}\right| \leq c(x, \xi, t, k)} d s=\int_{-c(x, \xi, t, k)}^{c(x, \xi, t, k)} d z L_{t / 2}^{z}
$$

where $L_{t / 2}^{z}$ stands for the local time at level $z$ and time $t / 2$ of the process $\left(x_{1} \frac{t-s}{t}+\xi_{1} \frac{s}{t}+\right.$ $\left.B_{s}^{0, t}\right)_{s \in[0, t]}$. From the definition of $P_{1}$ in (4.35):

$$
\begin{aligned}
P_{1} & \leq \mathbb{P}\left[\sup _{z \in[-c(x, \xi, t, k), c(x, \xi, t, k)]} L_{t / 2}^{z} \times 2 c(x, \xi, t, k)>\frac{t}{8}\right] \\
& =\mathbb{P}\left[\sup _{z \in\left[-\frac{c(x, \xi, t, k)}{t^{1 / 2}}, \frac{c(x, \xi, t, k)}{t^{1 / 2}}\right]} \bar{L}_{1 / 2}^{z}>\frac{t^{1 / 2}}{16 c(x, \xi, t, k)}\right],
\end{aligned}
$$

where $\bar{L}_{1 / 2}^{z}$ stands for the local time at level $z$ and time $1 / 2$ for the scalar process

$$
\left(\bar{X}_{u}\right)_{u \in[0,1]}:=\left(\frac{x_{1}}{t^{1 / 2}}(1-u)+\frac{\xi_{1}}{t^{1 / 2}} u+\frac{B_{u t}^{0, t}}{t^{1 / 2}}\right)_{u \in[0,1]} \stackrel{(\text { law })}{=}\left(\frac{x_{1}}{t^{1 / 2}}(1-u)+\frac{\xi_{1}}{t^{1 / 2}} u+B_{u}^{0,1}\right)_{u \in[0,1]} .
$$

The last equality in (4.36) is a consequence of the scaling properties of the local time. From Tanaka's formula for semimartingales $\bar{L}_{1 / 2}^{z}=\left|\bar{X}_{1 / 2}-z\right|-\left|\bar{X}_{0}-z\right|-\int_{0}^{1 / 2} \operatorname{sgn}\left(\bar{X}_{s}-z\right) d \bar{X}_{s}$. 
Denoting with a slight abuse of notation $\left(\frac{B_{u t}^{0, t}}{t^{1 / 2}}\right)_{u \in[0,1]}=\left(B_{u}^{0,1}\right)_{u \in[0,1]}$, we have the following differential dynamics for $\bar{X}_{u}$ :

$$
d \bar{X}_{u}=-\frac{x_{1}-\xi_{1}}{t^{1 / 2}} d u+d B_{u}^{0,1}=-\frac{\bar{X}_{u}-\xi_{1}}{1-u} d u+d B_{u}
$$

where $\left(B_{u}\right)_{u \in[0,1]}$ is a standard scalar Brownian motion.

Therefore, from equation (4.36) and the usual differential dynamics for the Brownian bridge:

$$
\begin{aligned}
& P_{1} \leq \mathbb{P}\left[\frac{\left|\xi_{1}-x_{1}\right|}{2 t^{1 / 2}}+\left|B_{1 / 2}^{0,1}\right|\right. \\
& \left.+\sup _{z \in\left[-\frac{c(x, \xi, t, k)}{t^{1 / 2}}, \frac{c(x, \xi, t, k)}{t^{1 / 2}}\right]}\left|\int_{0}^{1 / 2} \operatorname{sgn}\left(\bar{X}_{s}-z\right)\left(-\frac{x_{1}-\xi_{1}}{t^{1 / 2}} d s+d B_{s}^{0,1}\right)\right| \geq \frac{t^{1 / 2}}{8 c(x, \xi, t, k)}\right] \\
& \leq \mathbb{P}\left[\frac{\left|\xi_{1}-x_{1}\right|}{t^{1 / 2}}+\left|B_{1 / 2}^{0,1}\right|+\right. \\
& \left.\int_{0}^{1 / 2} d s \frac{\left|B_{s}^{0,1}\right|}{1-s}+\sup _{z \in\left[-\frac{c(x, \xi, t, k)}{t^{1 / 2}}, \frac{c(x, \xi, t, k)}{t^{1 / 2}}\right]}\left|\int_{0}^{1 / 2} \operatorname{sgn}\left(\bar{X}_{s}-z\right) d B_{s}\right| \geq \frac{t^{1 / 2}}{16 c(x, \xi, t, k)}\right] \\
& \leq \mathbb{P}\left[2 K+3 \sup _{s \in[0,1 / 2]}\left|B_{s}^{0,1}\right|+\sup _{z \in\left[-\frac{c(x, \xi, t, k)}{t^{1 / 2}}, \frac{c(x, \xi, t, k)}{t^{1 / 2}}\right]}\left|\int_{0}^{1 / 2} \operatorname{sgn}\left(\bar{X}_{s}-z\right) d B_{s}\right| \geq \frac{t^{1 / 2}}{16 c(x, \xi, t, k)}\right] .
\end{aligned}
$$

Now from the definition of $c(x, \xi, t, k)$ in $(4.35)$, for $t \geq\left[\left(\xi_{n+1}-x_{x+1}\right) \frac{3}{4}(64 K)^{k}\right]^{2 /(k+2)}$ one has $\frac{t^{1 / 2}}{16 c(x, \xi, t, k)}-2 K \geq \frac{t^{1 / 2}}{32 c(x, \xi, t, k)}$. Thus

$$
\begin{aligned}
& P_{1} \leq \mathbb{P}\left[3 \sup _{s \in[0,1 / 2]}\left|B_{s}^{0,1}\right|\right.\left.\geq \frac{t^{1 / 2}}{64 c(x, \xi, t, k)}\right] \\
&+\mathbb{P}\left[\sup _{z \in\left[-\frac{c(x, \xi, t, k)}{t^{1 / 2}}, \frac{c(x, \xi, t, k)}{t^{1 / 2}}\right]}\left|\int_{0}^{1 / 2} \operatorname{sgn}\left(\bar{X}_{s}-z\right) d B_{s}\right| \geq \frac{t^{1 / 2}}{64 c(x, \xi, t, k)}\right] .
\end{aligned}
$$

Setting for all $t \in[0,1 / 2], M_{t}:=\int_{0}^{t} \operatorname{sgn}\left(\bar{X}_{s}-z\right) d B_{s}, M_{t}:=\widetilde{B}_{\langle M\rangle_{t}}=\widetilde{B}_{t}$ (i.e. $\widetilde{B}$ is the Dambis-Dubbins-Schwarz Brownian motion associated to $M$ ). Hence, from Proposition 4.2 we derive the announced bound for $P_{1}$. Since $P_{2}$ can be handled in a similar way, the claim then follows from equation (4.35).

\subsection{Final derivation of the upper-bounds in the various regimes}

In this section we put together our previous estimates in order to derive the upper bounds of Theorem 2.1 in the various regimes. 


\subsubsection{Derivation of the Gaussian upper bounds}

In this paragraph we assume $\left|x_{1, n}\right| \vee\left|\xi_{1, n}\right| \geq K t^{1 / 2}$ for $K$ large enough. We also suppose $\frac{\left|\xi_{n+1}-x_{n+1}-c t\left(\left|x_{1, n}\right|^{k}+\left|\xi_{1, n}\right|^{k}\right)\right|}{t^{3 / 2}\left(\left|x_{1, n}\right|^{k-1}+\left|\xi_{1, n}\right|^{k-1}\right)} \leq \bar{C}$ where $c:=c(k)=2+\frac{2^{k-1}}{k+1}$ and $\bar{C}$ is fixed. From Corollary 3.7 (representation of the density), Proposition 4.6 (controls of the weight in the integration by part) and Lemma 4.8 (deviation bounds), we have that there exists $C:=C(n, k, K, \bar{C}) \geq 1$, s.t. setting $U_{t}^{k}(x, \xi):=\xi_{n+1}-x_{n+1}-\frac{2^{k-1}}{k+1}\left(\left|x_{1, n}\right|^{k}+\left|\xi_{1, n}\right|^{k}\right) t$ as in Lemma 4.8 one has:

$$
p(t, x, \xi) \leq \frac{C \exp \left(-\frac{\left|\xi_{1, n}-x_{1, n}\right|^{2}}{2 t}-C^{-1} \frac{U_{t}^{k}(x, \xi)^{2}}{\left(\left|x_{1, n}\right|^{k-1}+\left|\xi_{1, n}\right|^{k-1}\right)^{2} t^{3}}\right)}{t^{n / 2+3 / 2}\left(\left|x_{1, n}\right|^{k-1}+\left|\xi_{1, n}\right|^{k-1}\right)} .
$$

Remark 4.10 The above result means that the Gaussian regime holds if the final point $\xi_{1, n}$ of the degenerate component has the same order as the "mean" transport term $m_{t}(x, \xi):=$ $x_{n+1}+\frac{2^{k-1}}{k+1}\left(\left|x_{1, n}\right|^{k}+\left|\xi_{1, n}\right|^{k}\right) t$ (moderate deviations). A similar lower bound holds true, see Lemma 4.11.

\subsubsection{Derivation of the heavy-tailed upper bounds}

We here assume $\frac{\left|\xi_{n+1}-x_{n+1}-c t\left(\left|x_{1, n}\right|^{k}+\left|\xi_{1, n}\right|^{k}\right)\right|}{t^{3 / 2}\left(\left|x_{1, n}\right|^{k-1}+\left|\xi_{1, n}\right|^{k-1}\right)} \geq \bar{C}$ where $c:=c(k)=2+\frac{2^{k-1}}{k+1}$ and $\bar{C}$ is as in the previous paragraph.

If $\left|x_{1, n}\right| \vee\left|\xi_{1, n}\right| \leq K t^{1 / 2}$ ( $K$ being as in the previous paragraph), then Corollary 3.7, Proposition 4.7 and Lemma 4.8 yield that there exists $C:=C(n, k) \geq 1$ s.t.

$$
p(t, x, \xi) \leq \frac{C}{t^{(n+k) / 2+1}} \exp \left(-\frac{\left|\xi_{1, n}-x_{1, n}\right|^{2}}{2 t}-C^{-1} \frac{\left(U_{t}^{k}(x, \xi)\right)^{2 / k}}{t^{1+2 / k}}\right) .
$$

On the other hand if $\left|x_{1, n}\right| \vee\left|\xi_{1, n}\right| \geq K t^{1 / 2}$, then Corollary 3.7, Proposition 4.6 and Lemma 4.8 yield that there exists $\widetilde{C}:=\widetilde{C}(n, k) \geq 1$ s.t.

$$
\begin{aligned}
p(t, x, \xi) & \leq \frac{\widetilde{C}}{t^{n / 2+3 / 2}\left(\left|x_{1, n}\right|^{k-1}+\left|\xi_{1, n}\right|^{k-1}\right)} \exp \left(-\frac{\left|\xi_{1, n}-x_{1, n}\right|^{2}}{2 t}-\widetilde{C}^{-1} \frac{U_{t}^{k}(x, \xi)^{2 / k}}{t^{1+2 / k}}\right) \\
& \leq \frac{\widetilde{C}}{K^{k-1} t^{(n+k) / 2+1}} \exp \left(-\frac{\left|\xi_{1, n}-x_{1, n}\right|^{2}}{2 t}-\widetilde{C}^{-1} \frac{U_{t}^{k}(x, \xi)^{2 / k}}{t^{1+2 / k}}\right) .
\end{aligned}
$$

Hence, up to a modification of $C$, the control given by (4.38) holds for all off-diagonal cases.

\subsubsection{Moderate deviations of the degenerate component}

In this paragraph we suppose $0<\xi_{n+1}-x_{n+1} \leq K t^{1+k / 2}$, for $K$ sufficiently small. This means that the deviation of the degenerate component is small w.r.t. its characteristic time scale. From Corollary 3.7, Propositions 4.6 and 4.7 and Proposition 4.9 we derive similarly to the previous paragraph that there exists $C:=C(n, k, K)$ s.t.

$$
p(t, x, \xi) \leq \frac{C \exp \left(-\frac{\left|\xi_{1, n}-x_{1, n}\right|^{2}}{2 t}-C^{-1}\left\{\frac{\left|x_{1, n}\right|^{2+k}+\left|\xi_{1, n}\right|^{2+k}}{\xi_{n+1}-x_{n+1}}+\frac{t^{1+2 / k}}{\left(\xi_{n+1}-x_{n+1}\right)^{2 / k}}\right\}\right)}{t^{(n+k) / 2+1}} .
$$




\subsection{Gaussian lower bound on the compact sets of the metric}

We conclude this section with a proof of a lower bound for the density on the compact sets of the metric associated to the Gaussian regime in Theorem 2.1. A similar feature already appears in the appendix of [19].

Lemma 4.11 Assume that $\left|x_{1, n}\right| \vee\left|\xi_{1, n}\right| \geq K t^{1 / 2}, K \geq K_{0}:=K_{0}(n, k)$ and that for a given $\bar{C} \geq 0$ we have $\frac{\left|\xi_{n+1}-x_{n+1}-c t\left(\left|x_{1, n}\right|^{k}+\left|\xi_{1, n}\right|^{k}\right)\right|}{t^{3 / 2}\left(\left|x_{1, n}\right|^{k-1}+\left|\xi_{1, n}\right|^{k-1}\right)} \leq \bar{C}$ where $c:=c(k)$ is fixed. Then, there exists $C_{4.11}:=C_{4.11}(n, k, \bar{C})$ s.t.

$$
\frac{C_{4.11}}{\left(\left|x_{1, n}\right|^{k-1}+\left|\xi_{1, n}\right|^{k-1}\right) t^{3 / 2}} \leq p_{Y_{t}}\left(\xi_{n+1}-x_{n+1}\right) .
$$

Remark 4.12 The condition in the Lemma means that the deviation $\xi_{n+1}-x_{n+1}$ has exactly the same order as the transport term $t\left(\left|x_{1, n}\right|^{k}+\left|\xi_{1, n}\right|^{k}\right)$, up to a neglectable fluctuation corresponding to the variance of the Gaussian contribution in $Y_{t}$.

Proof. We assume w.l.o.g. that $\xi_{n+1}-x_{n+1}-\operatorname{ct}\left(\left|x_{1, n}\right|^{k}+\left|\xi_{1, n}\right|^{k}\right) \geq 0$ and $\left|\xi_{1, n}\right| \geq\left|x_{1, n}\right|$. From (4.19) we recall:

$$
\begin{aligned}
p_{Y_{t}}\left(\xi_{n+1}-x_{n+1}\right) & =\mathbb{E}\left[H_{t} \mathbb{I}_{Y_{t} \geq \xi_{n+1}-x_{n+1}}\right], \\
H_{t} & :=H_{t}^{1}+H_{t}^{2}:=\gamma_{Y_{t}}^{-2}\left\langle D \gamma_{Y_{t}}, D Y_{t}\right\rangle_{L^{2}(0, t)}+\Gamma_{Y_{t}} L Y_{t} .
\end{aligned}
$$

Recalling the chaos decomposition of $L Y_{t}$ introduced in Proposition 4.6, see equation (4.25), we get:

$$
\begin{aligned}
& p_{Y_{t}}\left(\xi_{n+1}-x_{n+1}\right) \geq \mathbb{E}\left[H_{t}^{2} \mathbb{I}_{Y_{t} \geq \xi_{n+1}-x_{n+1}}\right]-\mathbb{E}\left[\left|H_{t}^{1}\right|\right] \geq \mathbb{E}\left[\frac{I_{1}\left(g_{0}(., t)\right.}{\gamma_{Y_{t}}} \mathbb{I}_{Y_{t} \geq \xi_{n+1}-x_{n+1}}\right] \\
& -\left\{\mathbb{E}\left[\frac{\left|\sum_{l=2}^{k} I_{l}\left(g_{l-1}(., t)\right)\right|}{\gamma_{Y_{t}}}\right]+\frac{\bar{C}_{1}}{t\left|\xi_{1, n}\right|^{k}}\right\},
\end{aligned}
$$

using the bound for $\mathbb{E}\left[\left|H_{t}^{1}\right|\right]$ given by equation (4.23), with $\bar{C}_{1}:=\bar{C}_{1}(n, k, 1, K)$, in the last inequality. From equation (4.26), there exists $\bar{C}_{2}:=\bar{C}_{2}(n, k), \mathbb{E}\left[\left|\sum_{l=2}^{k} I_{l}\left(g_{l-1}(., t)\right)\right|^{2}\right]^{1 / 2} \leq$ $\bar{C}_{2} t^{3 / 2}\left|\xi_{1, n}\right|^{k-1} \sum_{l=2}^{k}\left(\frac{t^{1 / 2}}{\left|\xi_{1, n}\right|}\right)^{l-1} \leq \frac{(k-1) \bar{C}_{2}}{K} t^{3 / 2}\left|\xi_{1, n}\right|^{k-1}$, recalling $\left|\xi_{1, n}\right| \geq K t^{1 / 2}$ for the last inequality. Also $I_{1}\left(g_{0}(., t)\right)=\int_{0}^{t} \mathbb{E}\left[\bar{M}_{1}(u, t)\right] d W_{u}+R_{0}^{t}$ where $\mathbb{E}\left[\left|R_{0}^{t}\right|^{2}\right]^{1 / 2} \leq \frac{\bar{C}_{2}}{K} t^{3 / 2}\left|\xi_{1, n}\right|^{k-1}$. From (4.5), we write:

$$
\begin{aligned}
Y_{t}= & \int_{0}^{t} d u\left|m\left(u, t, x_{1, n}, \xi_{1, n}\right)\right|^{k}+k \int_{0}^{t} d u\left|m\left(u, t, x_{1, n}, \xi_{1, n}\right)\right|^{k-2}\left\langle m\left(u, t, x_{1, n}, \xi_{1, n}\right), W_{u}^{0, t}\right\rangle \\
& +R_{t}^{k}\left(x_{1, n}, \xi_{1, n}\right)=:\left(m_{t}^{k}+G_{t}^{k}+R_{t}^{k}\right)\left(x_{1, n}, \xi_{1, n}\right)=: m_{t}^{k}+G_{t}^{k}+R_{t}^{k},
\end{aligned}
$$

for simplicity. Proposition 4.6 then yields:

$$
\begin{aligned}
p_{Y_{t}}\left(\xi_{n+1}-x_{n+1}\right) \geq & \mathbb{E}\left[\frac{\int_{0}^{t} \mathbb{E}\left[\bar{M}_{1}(u, t)\right] d W_{u}}{\gamma_{Y_{t}}} \mathbb{I}_{m_{t}^{k}+G_{t}^{k}+R_{t}^{k} \geq \xi_{n+1}-x_{n+1}}\right] \\
& -\left\{\frac{C_{2} \bar{C}_{2} k t^{3 / 2}\left|\xi_{1, n}\right|^{k-1}}{K\left|\xi_{1, n}\right|^{2(k-1)} t^{3}}+\frac{\bar{C}_{1}}{K t^{3 / 2}\left|\xi_{1, n}\right|^{k-1}}\right\} \\
:= & p_{Y_{t}, 1}\left(\xi_{n+1}-x_{n+1}\right)-r_{1}(t, x, \xi) .
\end{aligned}
$$


From the martingale representation theorem and the above computations we identify $G_{t}^{k}=$ $\int_{0}^{t} \mathbb{E}\left[\bar{M}_{1}(u, t)\right] d W_{u}$. Still from Proposition 4.6 we get:

$$
\begin{aligned}
p_{Y_{t}}\left(\xi_{n+1}-x_{n+1}\right) \geq & \mathbb{E}\left[\frac{G_{t}^{k}}{\gamma_{Y_{t}}} \mathbb{I}_{G_{t}^{k}+R_{t}^{k} \geq \xi_{n+1}-x_{n+1}-m_{t}^{k}} \mathbb{I}_{\left|R_{t}^{k}\right| \leq\left|G_{t}^{k}\right| / 2}\right] \\
& -\left[\frac{\bar{C}_{3} \mathbb{P}\left[\left|R_{t}^{k}\right|>\left|G_{t}^{k}\right| / 2\right]^{1 / 2}}{\left(\left|x_{1, n}\right|^{k-1}+\left|\xi_{1, n}\right|^{k-1}\right) t^{3 / 2}}+r_{1}(t, x, \xi)\right] \\
= & p_{Y_{t}, 2}\left(\xi_{n+1}-x_{n+1}\right)-r_{2}(t, x, \xi),
\end{aligned}
$$

where $\bar{C}_{3}:=\bar{C}_{3}(n, k)$. One easily gets that there exists $c:=c(k)>0, m_{t}^{k}:=m_{t}^{k}\left(x_{1, n}, \xi_{1, n}\right) \geq$ $c t\left(\left|x_{1, n}\right|^{k}+\left|\xi_{1, n}\right|^{k}\right)$.Thus, setting $U_{t}^{k}(x, \xi):=\xi_{n+1}-x_{n+1}-c t\left(\left|x_{1, n}\right|^{k}+\left|\xi_{1, n}\right|^{k}\right)$ and recalling as well that $U_{t}^{k}(x, \xi) \geq 0$, one obtains that on the event $\left\{G_{t}^{k}+R_{t}^{k} \geq U_{t}^{k}(x, \xi),\left|R_{t}^{k}\right| \leq\left|G_{t}^{k}\right| / 2\right\}$, $G_{t}^{k} \geq 0$. Hence:

$$
\begin{aligned}
p_{Y_{t}}\left(\xi_{n+1}-x_{n+1}\right) \geq & \mathbb{E}\left[\frac{G_{t}^{k}}{\gamma_{Y_{t}}} \mathbb{I}_{G_{t}^{k}-\left|R_{t}^{k}\right| \geq U_{t}^{k}(x, \xi) \geq 0} \mathbb{I}_{\left|R_{t}^{k}\right| \leq\left|G_{t}^{k}\right| / 2}\right]-r_{2}(t, x, \xi) \\
\geq & \mathbb{E}\left[\frac{G_{t}^{k}}{\gamma_{Y_{t}}} \mathbb{I}_{G_{t}^{k} \geq 2 U_{t}^{k}(x, \xi) \geq 0} \mathbb{I}_{\left|R_{t}^{k}\right| \leq G_{t}^{k} / 2}\right]-r_{2}(t, x, \xi) \\
\geq & \mathbb{E}\left[\frac{G_{t}^{k}}{3 \mathcal{M}_{t}} \mathbb{I}_{G_{t}^{k} \geq 2 U_{t}^{k}(x, \xi) \geq 0} \mathbb{I}_{\left|R_{t}^{k}\right| \leq G_{t}^{k} / 2} \mathbb{I}_{\gamma_{Y_{t}} \leq 3 \mathcal{M}_{t}}\right]-\frac{\bar{C}_{3} \mathbb{P}\left[\gamma_{Y_{t}}>3 \mathcal{M}_{t}\right]^{1 / 2}}{\left|\xi_{1, n}\right|^{k-1} t^{3 / 2}} \\
& -r_{2}(t, x, \xi) \\
\geq & \mathbb{E}\left[\frac{G_{t}^{k}}{3 \mathcal{M}_{t}} \mathbb{I}_{G_{t}^{k} \geq 2 \bar{C} t^{3 / 2}\left(\left|x_{1, n}\right|^{k-1}+\left|\xi_{1, n}\right|^{k-1}\right)\left|R_{t}^{k}\right| \leq G_{t}^{k} / 2} \mathbb{I}_{\gamma_{Y_{t}} \leq 3 \mathcal{M}_{t}}\right]-r_{3}(t, x, \xi) \\
\geq & \frac{C^{-1} \bar{C} \bar{P} \mathbb{P}\left[G_{t}^{k} \geq 2 \bar{C} t^{3 / 2}\left(\left|x_{1, n}\right|^{k-1}+\left|\xi_{1, n}\right|^{k-1}\right),\left|R_{t}^{k}\right| \leq G_{t}^{k} / 2\right]}{3 t^{3 / 2}\left(\left|x_{1, n}\right|^{k-1}+\left|\xi_{1, n}\right|^{k-1}\right)} \\
& -\frac{\bar{C}_{4} \mathbb{P}\left[\gamma_{Y_{t}}>3 \mathcal{M}_{t}\right]^{1 / 2}}{t^{3 / 2}\left|\xi_{1, n}\right|^{k-1}}-r_{3}(t, x, \xi),
\end{aligned}
$$

where we used that $U_{t}^{k}(x, \xi) \leq \bar{C} t^{3 / 2}\left(\left|x_{1, n}\right|^{k-1}+\left|\xi_{1, n}\right|^{k-1}\right)$ for the last but one inequality (compact sets of the metric). The constant $C$ is the one appearing in (4.10). To conclude it suffices to prove that

$$
\begin{aligned}
P & \left.:=\mathbb{P}\left[G_{t}^{k} \geq 2 C t^{3 / 2}\left(\left|x_{1, n}\right|^{k-1}+\left|\xi_{1, n}\right|^{k-1}\right),\left|R_{t}^{k}\right| \leq\left|G_{t}^{k}\right| / 2\right]\right] \geq \widetilde{C} \\
\left|r_{4}(t, x, \xi)\right| & :=\frac{\bar{C}_{4} \mathbb{P}\left[\gamma_{Y_{t}}>3 \mathcal{M}_{t}\right]^{1 / 2}}{t^{3 / 2}\left|\xi_{1, n}\right|^{k-1}}+r_{3}(t, x, \xi) \leq \frac{C^{-1} \bar{C} \widetilde{C}}{3 t^{3 / 2}\left(\left|x_{1, n}\right|^{k-1}+\left|\xi_{1, n}\right|^{k-1}\right)}
\end{aligned}
$$

Indeed, plugging (4.41) and (4.42) into (4.40) gives the statement. Let us first prove (4.41). Write:

$$
\begin{aligned}
P \geq & \mathbb{P}\left[G_{t}^{k} \geq 2 C t^{3 / 2}\left(\left|x_{1, n}\right|^{k-1}+\left|\xi_{1, n}\right|^{k-1}\right)\right] \\
& -\mathbb{P}\left[G_{t}^{k} \geq 2 C t^{3 / 2}\left(\left|x_{1, n}\right|^{k-1}+\left|\xi_{1, n}\right|^{k-1}\right),\left|R_{t}^{k}\right|>\left|G_{t}^{k}\right| / 2\right] \\
\geq & \mathbb{P}[\mathcal{N}(0,1) \geq 2 \check{C}]-\mathbb{P}\left[\left|R_{t}^{k}\right| \geq C t^{3 / 2}\left(\left|x_{1, n}\right|^{k-1}+\left|\xi_{1, n}\right|^{k-1}\right)\right], \check{C}:=\check{C}(n, k) .
\end{aligned}
$$


Thus, similarly to the proof of (4.12) in Lemma 4.3 we can show that there exists $\bar{C}_{5}:=$ $\bar{C}_{5}(n, k) \geq 1$ s.t. $\mathbb{P}\left[\left|R_{t}^{k}\right| \geq C t^{3 / 2}\left(\left|x_{1, n}\right|^{k-1}+\left|\xi_{1, n}\right|^{k-1}\right)\right] \leq \bar{C}_{5} \exp \left(-\bar{C}_{5}^{-1} \frac{\left|x_{1, n}\right|^{2}+\left|\xi_{1, n}\right|^{2}}{t}\right)$. Under the current assumptions, using standard controls on the Gaussian distribution function, this gives (4.41) for $\widetilde{C}:=\widetilde{C}(n, k)$ for $K$ large enough.

Recall now that $\left|r_{4}(t, x, \xi)\right| \leq \frac{1}{t^{3 / 2}\left|\xi_{1, n}\right|^{k-1}}\left(\frac{\bar{C}_{1}+C_{2} \bar{C}_{2} k}{K}+\left(\bar{C}_{3}+\bar{C}_{4}\right) \mathbb{P}\left[\gamma_{Y_{t}}>3 \mathcal{M}_{t}\right]^{1 / 2}+\right.$ $\left.\bar{C}_{3} \mathbb{P}\left[\left|R_{t}^{k}\right|>\left|G_{t}^{k}\right| / 2\right]^{1 / 2}\right):=\sum_{i=1}^{3} r_{4 i}(t, x, \xi)$. Under the current assumptions, we derive that for $K$ large enough, $r_{41}(t, x, \xi) \leq \frac{C^{-1} \bar{C} \widetilde{C}}{9 t^{3 / 2}\left(\left|x_{1, n}\right|^{k-1}+\left|\xi_{1, n}\right|^{k-1}\right)}$. On the other hand, writing $\mathbb{P}\left[\left|R_{t}^{k}\right|>\right.$ $\left.\left|G_{t}^{k}\right| / 2\right]^{1 / 2} \leq\left(\mathbb{P}\left[\left|R_{t}^{k}\right|>\frac{\hat{C}}{2}\left(\left|x_{1, n}\right|^{k-1}+\left|\xi_{1, n}\right|^{k-1}\right) t^{3 / 2}\right]+\mathbb{P}\left[\left|G_{t}^{k}\right| \leq \hat{C}\left(\left|x_{1, n}\right|^{k-1}+\left|\xi_{1, n}\right|^{k-1}\right) t^{3 / 2}\right]\right)^{1 / 2}$ we derive similarly to (4.12) (see also the proof of the lower bound in Lemma 4.5) that $r_{43}(t, x, \xi) \leq \frac{C^{-1} \bar{C} \widetilde{C}}{9 t^{3 / 2}\left(\left|x_{1, n}\right|^{k-1}+\left|\xi_{1, n}\right|^{k-1}\right)}$ taking $\hat{C}$ small enough. Eventually, the same control holds true for $r_{42}(t, x, \xi)$, still from arguments similar to those used to derive (4.12). This concludes the proof.

\section{$5 \quad$ Potential Theory and PDEs}

In this section we are interested in proving Harnack inequalities for non-negative solutions to

$$
\mathscr{L} u(z)=0, \quad z=(x, t) \in \mathbb{R}^{N+1},
$$

with $\mathscr{L}$ defined in (1.2). Specifically, we consider any open set $\mathcal{O} \subseteq \mathbb{R}^{N+1}$, and any $z \in \mathcal{O}$, and we aim to show that there exists a compact $\mathcal{K} \subset \mathcal{O}$ and a positive constant $C_{\mathcal{K}}$ such that

$$
\sup _{\mathcal{K}} u \leq C_{\mathcal{K}} u(z)
$$

for every positive solution $u$ to $\mathscr{L} u=0$. We say that a set $\left\{z_{0}, z_{1}, \ldots, z_{k}\right\} \subset \mathcal{O}$ is a Harnack chain of lenght $k$ if

$$
u\left(z_{j}\right) \leq C_{j} u\left(z_{j-1}\right), \quad \text { for } j=1, \ldots, k,
$$

for every positive solution $u$ of $\mathscr{L} u=0$, so that we get

$$
u\left(z_{k}\right) \leq C_{1} C_{2} \ldots C_{k} u\left(z_{0}\right)
$$

In order to construct Harnack chains, and to have an explicit lower bound for the densities considered in this article, we will prove invariant Harnack inequalities w.r.t. a suitable Lie group structure. By exploiting the properties of homogeneity and translation invariance of the Lie group, we will find Harnack chains with the property that every $C_{j}$ in (5.3) agrees with the constant $C_{\mathcal{K}}$ in (5.2). As a consequence we find $u\left(z_{k}\right) \leq C_{\mathcal{K}}^{k} u\left(z_{0}\right)$, and the bound will depend only on the lenght of the Harnack chain connecting $z_{0}$ to $z_{k}$.

Let us now recall some basic notations concerning homogeneous Lie groups (we refer to the monograph [7] by Bonfiglioli, Lanconelli and Uguzzoni for an exhaustive treatment). Let $\circ$ be a given group law on $\mathbb{R}^{N+1}$ and suppose that the map $(z, \zeta) \mapsto \zeta^{-1} \circ z$ is smooth. Then 
$\mathbb{G}=\left(\mathbb{R}^{N+1}, \circ\right)$ is called a Lie group. Moreover, $\mathbb{G}$ is said homogeneous if there exists a family of dilations $\left(\delta_{\lambda}\right)_{\lambda>0}$ which defines an automorphism of the group, i.e.,

$$
\delta_{\lambda}(z \circ \zeta)=\left(\delta_{\lambda} z\right) \circ\left(\delta_{\lambda} \zeta\right), \quad \text { for all } z, \zeta \in \mathbb{R}^{N+1} \text { and } \lambda>0 .
$$

We also make the following assumption.

$[\mathbf{L}] \mathscr{L}$ is Lie-invariant with respect to the Lie group $\mathbb{G}=\left(\mathbb{R}^{N+1}, \circ,\left(\delta_{\lambda}\right)_{\lambda>0}\right)$, i.e.

i) $Y_{1}, \ldots, Y_{n}$ and $Z$ are left-invariant with respect to the composition law of $\mathbb{G}$, i.e.

$$
\begin{aligned}
Y_{j}(u(\zeta \circ \cdot)) & =\left(Y_{j} u\right)(\zeta \circ \cdot), \quad j=1, \ldots, n, \\
Z(u(\zeta \circ \cdot)) & =(Z u)(\zeta \circ \cdot),
\end{aligned}
$$

for every function $u \in C^{\infty}\left(\mathbb{R}^{N+1}\right)$, and for any $\zeta \in \mathbb{R}^{N+1}$;

ii) $Y_{1}, \ldots, Y_{n}$ are $\delta_{\lambda}$-homogeneous of degree one and $Z$ is $\delta_{\lambda}$-homogeneous of degree two:

$$
\begin{aligned}
Y_{j}\left(u\left(\delta_{\lambda} z\right)\right) & =\lambda\left(Y_{j} u\right)\left(\delta_{\lambda} z\right), \quad j=1, \ldots, n, \\
Z\left(u\left(\delta_{\lambda} z\right)\right) & =\lambda^{2}(Z u)\left(\delta_{\lambda} z\right),
\end{aligned}
$$

for every function $u \in C^{\infty}\left(\mathbb{R}^{N+1}\right)$, and for any $z \in \mathbb{R}^{N+1}, \lambda>0$.

To illustrate Property $[\mathbf{L}]$ we recall the Lie group structure of the Kolmogorov operator corresponding to $k=1$ in (1.4).

Example 5.1 (Kolmogorov operators) $\mathscr{L}:=\frac{1}{2} \Delta_{x_{1, n}}+\sum_{i=1}^{n} x_{i} \partial_{x_{2}}-\partial_{t}$. The Kolmogorov group is $\mathbb{K}=\left(\mathbb{R}^{n+2}, \circ, \delta_{\lambda}\right)$, where

$$
(x, t) \circ(\xi, \tau)=\left(x_{1, n}+\xi_{1, n}, x_{n+1}+\xi_{n+1}-\sum_{i=1}^{n} x_{i} \tau, t+\tau\right), \quad \delta_{\lambda}(x, t)=\left(\lambda x_{1, n}, \lambda^{3} x_{n+1}, \lambda^{2} t\right) .
$$

Clearly, $\mathscr{L}$ can be written as in (1.2) with $Y_{i}=\partial_{x_{i}}$, $i \in \llbracket 1, n \rrbracket$, and $Z=\sum_{i=1}^{n} x_{i} \partial_{x_{n+1}}-\partial_{t}$, and satisfies $[\mathbf{L}]$.

It is known that the composition law $\circ$ is always a sum with respect to the $t$ variable (see Propostion 10.2 in [24]). Moreover, the family $\left(\delta_{\lambda}\right)_{\lambda>0}$ acts on $\mathbb{R}^{N+1}$ as follows:

$$
\delta_{\lambda}\left(x_{1}, x_{2}, \ldots, x_{N}, t\right)=\left(\lambda^{\sigma_{1}} x_{1}, \lambda^{\sigma_{2}} x_{2}, \ldots, \lambda^{\sigma_{N}} x_{N}, \lambda^{2} t\right), \quad \text { for every }(x, t) \in \mathbb{R}^{N+1},
$$

where $\sigma=\left(\sigma_{1}, \sigma_{2}, \ldots, \sigma_{N}\right) \in \mathbb{N}^{N}$ is a multi-index. The natural number $Q=\sum_{k=1}^{N} \sigma_{k}+2$ is called the homogeneous dimension of $\mathbb{G}$ with respect to $\delta_{\lambda}$. We shall assume that $Q \geq 3$. Observe that the diagonal decay of the heat kernel on the homogeneous Lie group is given by the characteristic time scale $t^{-(Q-2) / 2}$. For the above example we have $Q=n+3+2$, matching the diagonal exponent in $(1.5)(Q-2) / 2=(n+3) / 2$. 
Write the operator $\mathscr{L}$ as follows

$$
\mathscr{L}=\sum_{i, j=1}^{N} a_{i, j}(x) \partial_{x_{i}, x_{j}}+\sum_{j=1}^{N} b_{j}(x) \partial_{x_{j}}-\partial_{t},
$$

for suitable smooth coefficients $a_{i, j}$ 's and $b_{j}$ 's only depending on the vector fields $Y_{0}, \ldots, Y_{n}$. As $n<N, \mathscr{L}$ is strictly degenerate, since the $\operatorname{rank}(A(x)) \leq n$ at every $x$ (here $A(x):=$ $\left.\left(a_{i, j}(x)\right)_{i, j \in \llbracket 1, n \rrbracket}\right)$. In Example 5.1 we see that $\operatorname{rank}(A)$ never vanishes. We say that $\mathscr{L}$ is not totally degenerate if

[B] for every $x \in \mathbb{R}^{N}$ there exists $\nu \in \mathbb{R}^{N} \backslash\{0\}$ such that $\langle A(x) \nu, \nu\rangle>0$.

This property holds for a more general class of operators. Indeed, if $\mathscr{L}$ satisfies $[\mathbf{H}]$ and $[\mathbf{L}]$, then there exists a $\nu \in \mathbb{R}^{N} \backslash\{0\}$ such that

$$
\langle A(x) \nu, \nu\rangle>0, \quad \text { for every } x \in \mathbb{R}^{N} .
$$

We refer to Section 1.3 in the monograph [7] for the proof of this statement.

Fix now $T>0$ and define $I:=[0, T]$. We call diffusion trajectory any absolutely continuous curve on $I$ such that

$$
\gamma^{\prime}(s)=\sum_{k=1}^{n} \omega_{k}(s) Y_{k}(\gamma(s)), \quad \text { for every } s \in I,
$$

where $\omega_{1}, \ldots, \omega_{n}$ are piecewise constant real functions. A drift trajectory is any positively oriented integral curve of $Z$. We say that a curve $\gamma:[0, T] \rightarrow \mathbb{R}^{N+1}$ is $\mathscr{L}$-admissible if it is absolutely continuous and is a sum of a finite number of diffusion and drift trajectories.

Let $\mathcal{O}$ be any open subset of $\mathbb{R}^{N+1}$, and let $z_{0} \in \mathcal{O}$. We define the attainable set $\mathscr{A}_{z_{0}}:=\overline{A_{z_{0}}}$ as the closure in $\mathcal{O}$ of the following set

$$
\begin{aligned}
A_{z_{0}}=\{z \in \mathcal{O} & : \text { there exists an } \mathscr{L} \text {-admissible path } \\
& \left.\gamma:[0, T] \rightarrow \mathcal{O} \text { such that } \gamma(0)=z_{0}, \gamma(T)=z\right\} .
\end{aligned}
$$

The main result of the section is the following

Theorem 5.2 Let $\mathscr{L}$ be an operator in the form (5.1) satisfying $[\mathbf{H}]$ and $[\mathbf{L}]$, let $\mathcal{O} \subseteq \mathbb{R}^{N+1}$ be an open set, and let $z_{0} \in \mathcal{O}$. Then,

$$
\text { for every compact set } \mathcal{K} \subset \operatorname{Int}\left(\mathscr{A}_{z_{0}}\right), \quad \sup _{\mathcal{K}} u \leq C_{\mathcal{K}} u\left(z_{0}\right),
$$

for any non-negative solutions $u$ to $\mathscr{L} u=0$ in $\mathcal{O}$. Here $C_{\mathcal{K}}$ is a positive constant depending on $\mathcal{O}, \mathcal{K}, z_{0}$ and on $\mathscr{L}$.

We recall that a Harnack inequality for operators satisfying $[\mathbf{H}]$ and $[\mathbf{B}]$ is due to Bony (see [9]). Another result analogous to Theorem 5.2 is given in [15, Theorem 1.1] by Cinti, Nystrom and Polidoro, assuming $[\mathbf{L}]$ and the following controllability condition: 
[C] for every $(x, t),(\xi, \tau) \in \mathbb{R}^{N+1}$ with $t>\tau$, there exists an $\mathscr{L}$-admissible path $\gamma:[0, T] \rightarrow$ $\mathbb{R}^{N+1}$ such that $\gamma(0)=(x, t), \gamma(T)=(\xi, \tau)$.

Our Theorem 5.2 improves Bony's one in that it gives an explicit geometric description of the set $\mathcal{K}$ in (5.7). Also, it is more general than the one in $[15]$, since $[\mathbf{L}]$ and $[\mathbf{C}]$ imply $[\mathbf{H}]$ (see Proposition 10.1 in [24]).

The proof of Theorem 5.2 is based on a general result from Potential Theory. In Section 5.1 we recall the basic results of Potential Theory needed in our work, then we apply them to operators $\mathscr{L}$ satifying $[\mathbf{H}]$ and $[\mathbf{L}]$. We explicitly remark that condition $[\mathbf{L}]$ is not satisfied by the Kolmogorov operators

$$
\mathscr{L}=\frac{1}{2} \Delta_{x_{1, n}}+\left|x_{1, n}\right|^{k} \partial_{x_{n+1}}-\partial_{t}
$$

and

$$
\mathscr{L}=\frac{1}{2} \Delta_{x_{1, n}}+\sum_{j=1}^{n} x_{j}^{k} \partial_{x_{n+1}}-\partial_{t}
$$

of the stochastic systems (1.3) and (1.4) respectively. Indeed, in both cases $k$ commutators are needed to fulfill Hörmander condition $[\mathbf{H}]$ at $x_{1, n}=0$, while only one commutator is sufficient to span all the directions as $x_{1, n} \neq 0$, and this fact contradicts $\left.[\mathbf{L}]-i\right)$. On the other hand, the operators in (5.8) and (5.9) can be lifted to suitable operators $\widetilde{\mathscr{L}}=\widetilde{Y}_{1}^{2}+\widetilde{Z}$, satisfying both $[\mathbf{H}]$ and $[\mathbf{L}]$ (see (5.25)). We refer to Section 4 for more details, and we note that our Harnack-type inequality for $\mathscr{L}$, and the asymptotic lower bounds, are obtained in Section 4 by the application of Theorem 5.2 to $\widetilde{\mathscr{L}}$.

\subsection{Potential Theory}

For the first part of the section, we assume $\mathscr{L}$ to be a general abstract parabolic differential operator satisfying $[\mathbf{B}]$ and $[\mathbf{L}]$.

Let $\mathcal{O}$ be any open subset of $\mathbb{R}^{N+1}$. If $u: \mathcal{O} \rightarrow \mathbb{R}$ is a smoothfunction such that $\mathscr{L} u=0$ in $\mathcal{O}$, we say that $u$ is $\mathscr{L}$-harmonic in $\mathcal{O}$. We denote by $\mathcal{H}(\mathcal{O})$ the linear space of functions which are $\mathscr{L}$-harmonic in $\mathcal{O}$.

Let $V$ be a bounded open subset of $\mathbb{R}^{N+1}$ with Lipschitz-continuous boundary. We say that $V$ is $\mathscr{L}$-regular if, for every $z_{0} \in \partial V$, there exists a neighborhood $U$ of $z_{0}$ and a smooth function $w: U \rightarrow \mathbb{R}$ satisfying

$$
w\left(z_{0}\right)=0, \quad \mathscr{L} w\left(z_{0}\right)<0, \quad w>0 \text { in } \bar{V} \cap U \backslash\left\{z_{0}\right\} .
$$

Note that the function $\psi(x, t)=\frac{1}{2}+\frac{1}{\pi} \arctan t$ verifies

$$
0 \leq \psi \leq 1, \quad \mathscr{L} \psi<0 \text { in } \mathbb{R}^{N+1} .
$$

As a first consequence of (5.10), the classical Picone's maximum principle holds on any bounded open set $\mathcal{O} \subset \mathbb{R}^{N+1}$. Precisely, if $u \in C^{2}(\mathcal{O})$ satisfies

$$
\mathscr{L} u \geq 0 \quad \text { in } \mathcal{O}, \quad \limsup _{z \rightarrow \zeta} u(z) \leq 0 \quad \text { for every } \zeta \in \partial \mathcal{O},
$$


then $u \leq 0$ in $\mathcal{O}$ (see e.g. Bonfiglioli and Uguzzoni [8]). Then, for every $\mathscr{L}$-regular open set $V \subset \mathbb{R}^{N+1}$, and for any $\varphi \in C(\partial V)$ there exists a unique function $H_{\varphi}^{V}$ satisfying

$$
H_{\varphi}^{V} \in \mathcal{H}(V), \quad \lim _{z \rightarrow \zeta} H_{\varphi}^{V}(z)=\varphi(\zeta) \quad \text { for every } \zeta \in \partial V .
$$

Moreover, $H_{\varphi}^{V} \geq 0$ whenever $\varphi \geq 0$ (see Bauer [4] and Constantinescu and Cornea [18]). Hence, if $V$ is $\mathscr{L}$-regular, for every fixed $z \in V$ the map $\varphi \mapsto H_{\varphi}^{V}(z)$ defines a linear positive functional on $C(\partial V, \mathbb{R})$. Thus, the Riesz representation theorem implies that there exists a Radon measure $\mu_{z}^{V}$, supported in $\partial V$, such that

$$
H_{\varphi}^{V}(z)=\int_{\partial V} \varphi(\zeta) d \mu_{z}^{V}(\zeta), \quad \text { for every } \varphi \in C(\partial V, \mathbb{R})
$$

We will refer to $\mu_{z}^{V}$ as the $\mathscr{L}$-harmonic measure defined with respect to $V$ and $z$.

A lower semi-continuous function $u: \mathcal{O} \rightarrow]-\infty, \infty]$ is said to be $\mathscr{L}$-superharmonic in $\mathcal{O}$ if $u<\infty$ in a dense subset of $\mathcal{O}$ and if

$$
u(z) \geq \int_{\partial V} u(\zeta) d \mu_{z}^{V}(\zeta)
$$

for every open $\mathscr{L}$-regular set $V \subset \bar{V} \subset \mathcal{O}$ and for every $z \in V$. We denote by $\overline{\mathcal{S}}(\mathcal{O})$ the set of $\mathscr{L}$-superharmonic functions in $\mathcal{O}$, and by $\overline{\mathcal{S}}^{+}(\mathcal{O})$ the set of the functions in $\overline{\mathcal{S}}(\mathcal{O})$ which are non-negative. A function $v: \mathcal{O} \rightarrow[-\infty, \infty[$ is said to be $\mathscr{L}$-subharmonic in $\mathcal{O}$ if $-v \in \overline{\mathcal{S}}(\mathcal{O})$ and we write $\underline{\mathcal{S}}(\mathcal{O}):=-\overline{\mathcal{S}}(\mathcal{O})$. Since the collection of $\mathcal{L}$-regular sets is a basis for the Euclidean topology (as we will see in a moment), we have $\overline{\mathcal{S}}(\mathcal{O}) \cap \mathcal{S}(\mathcal{O})=\mathcal{H}(\mathcal{O})$.

This last property and Picone's maximum principle are the main tools in order to show the following criterion of $\mathscr{L}$-superharmonicity for functions of class $C^{2}$ (a proof can be found in the monograph [7, Proposition 7.2.5]).

Remark 5.3 Let $u \in C^{2}(\mathcal{O})$. Then $u$ is $\mathscr{L}$-superharmonic if and only if $\mathscr{L} u \leq 0$ in $\mathcal{O}$.

With the terminology of Potential Theory (we refer to the monographs $[4,18]$ ), the map $\mathbb{R}^{N+1} \supseteq \mathcal{O} \mapsto \mathcal{H}(\mathcal{O})$ is said harmonic sheaf and $\left(\mathbb{R}^{N+1}, \mathcal{H}\right)$ is said harmonic space. Since the constant functions are $\mathscr{L}$-harmonic, the last statement is a consequence of the following properties:

- the $\mathscr{L}$-regular sets form a basis for the Euclidean topology (by (5.4), $\mathscr{L}$ is a not totally degenerate operator, so that this statement is a consequence of [9, Corollaire 5.2]);

- $\mathcal{H}$ satisfies the Doob convergence property, i.e., the pointwise limit $u$ of any increasing sequence $\left\{u_{n}\right\}_{n}$ of $\mathscr{L}$-harmonic functions, on any open set $V$, is $\mathscr{L}$-harmonic whenever $u$ is finite in a dense set $T \subseteq V$ (as in [24, Proposition 7.4], we can rely on the weak Harnack inequality due to Bony stated in [9, Theoreme 7.1]);

- the family $\overline{\mathcal{S}}\left(\mathbb{R}^{N+1}\right)$ separates the points of $\mathbb{R}^{N+1}$, i.e., for every $z, \zeta \in \mathbb{R}^{N+1}, z \neq \zeta$, there exists $u \in \overline{\mathcal{S}}\left(\mathbb{R}^{N+1}\right)$ such that $u(z) \neq u(\zeta)$. 
This last separation property is proved in Lemma 5.5. We will in fact show a stronger result: actually, the family $\overline{\mathcal{S}}^{+}\left(\mathbb{R}^{N+1}\right) \cap C\left(\mathbb{R}^{N+1}\right)$ separates the points of $\mathbb{R}^{N+1}$. A harmonic space $\left(\mathbb{R}^{N+1}, \mathcal{H}\right)$ satisfying this property is said to be a $\mathfrak{B}$-harmonic space.

In order to prove the separation property we use a fundamental solution $\Gamma$ of $\mathscr{L}$. To prove the existence of a fundamental solution we now rely on condition $[\mathbf{H}]$ that we assumed to be in force through the paper. We recall that a fundamental solution is a function $\Gamma$ with the following properties:

i) the map $(z, \zeta) \mapsto \Gamma(z, \zeta)$ is defined, non-negative and smooth away from the set $\{(z, \zeta) \in$ $\left.\mathbb{R}^{N+1} \times \mathbb{R}^{N+1}: z \neq \zeta\right\}$

ii) for any $z \in \mathbb{R}^{N+1}, \Gamma(\cdot, z)$ and $\Gamma(z, \cdot)$ are locally integrable;

iii) for every $\phi \in C_{0}^{\infty}\left(\mathbb{R}^{N+1}\right)$ and $z \in \mathbb{R}^{N+1}$ we have

$$
\mathscr{L} \int_{\mathbb{R}^{N+1}} \Gamma(z, \zeta) \phi(\zeta) d \zeta=\int_{\mathbb{R}^{N+1}} \Gamma(z, \zeta) \mathscr{L} \phi(\zeta) d \zeta=-\phi(z) ;
$$

iv) $\mathscr{L} \Gamma(\cdot, \zeta)=-\delta_{\zeta}($ Dirac measure supported at $\zeta)$;

$v)$ if we define $\Gamma^{*}(z, \zeta):=\Gamma(\zeta, z)$, then $\Gamma^{*}$ is the fundamental solution for the formal adjoint $\mathscr{L}^{*}$ of $\mathscr{L}$, satisfying the dual statements of $\left.\left.i i i\right), i v\right)$;

vi) $\Gamma(x, t, \xi, \tau)=0$ if $t<\tau$.

Remark 5.4 Assumption $[\mathbf{H}]$ implies the existence of a smooth density $p(t, \xi, x) d x:=\mathbb{P}_{\xi}\left[X_{t} \in\right.$ $d x], t>0$, for the process $\left(X_{t}\right)_{t \geq 0}$ associated to $\mathscr{L}$ see e.g. Stroock [37] or Nualart [32]. Actually,

$$
\Gamma(x, t, \xi, \tau):=p(t-\tau, \xi, x)
$$

is a fundamental solution for $\mathscr{L}$ in the above sense. Indeed $p$ satisfies the Kolmogorov equation $\mathscr{L} p=0$, in $\mathbb{R}^{N+1} \backslash\{(\xi, \tau)\}$. We refer to Bonfiglioli and Lanconelli [6] for a purely analytic proof of existence of fundamental solutions for operators satisfying $[\mathbf{H}],[\mathbf{L}]$.

If condition [L] holds, then we also have:

vii) $\Gamma(z, \zeta)=\Gamma(\alpha \circ z, \alpha \circ \zeta)$ for every $\alpha, z, \zeta \in \mathbb{R}^{N+1}, z \neq \zeta$;

viii) $\Gamma\left(\delta_{\lambda}(z), \delta_{\lambda}(\zeta)\right)=\lambda^{-Q+2} \Gamma(z, \zeta), \quad z, \zeta \in \mathbb{R}^{N+1}, z \neq \zeta, \lambda>0$. 7.1].

We next prove the separation property for $\mathscr{L}$ by adapting the argument in [14, Proposition

Lemma 5.5 For every $z_{1}, z_{2} \in \mathbb{R}^{N+1}, z_{1} \neq z_{2}$, there exists a function $u \in \overline{\mathcal{S}}^{+}\left(\mathbb{R}^{N+1}\right) \cap$ $C\left(\mathbb{R}^{N+1}\right)$ such that $u\left(z_{1}\right) \neq u\left(z_{2}\right)$. 
Proof. Let us denote $z_{i}=\left(x_{i}, t_{i}\right)$ for $i=1,2$. First we suppose that $t_{1}<t_{2}$. The properties of $\Gamma$ yield that there exists $z_{0}=\left(x_{0}, t_{0}\right)$ with $t_{0}>0$ such that $\Gamma\left(z_{0}, 0\right)>0$. On the other hand, since $[\mathbf{H}]$ and $[\mathbf{L}]$ yield $[\mathbf{B}]$, there exists a $\mathscr{L}$-regular open set $V_{0}$ containing the origin, a small $r_{0}>0$ and a large $\lambda_{0}>1$ such that

$$
U_{r_{0}} \subseteq V_{0} \subseteq \delta_{\lambda_{0}}\left(U_{r_{0}}\right), \quad U_{r_{0}}=\left\{\left(x_{1}, \ldots, x_{N}, t\right) \in \mathbb{R}^{N+1}:\left|x_{i}\right|<r_{0},|t|<r_{0}\right\} .
$$

By the smoothness of $\Gamma$, there exists $\varepsilon>0$ such that $\Gamma>0$ in the set $z_{0} \circ U_{\varepsilon}$. For a fixed $\lambda \in] 0, \sqrt{\frac{t_{2}-t_{1}}{2\left(t_{0}+\varepsilon\right)}}\left[\right.$ and a non-negative function $\varphi \in C_{0}^{\infty}\left(z_{2} \circ\left(\delta_{\lambda}\left(z_{0} \circ U_{\varepsilon}\right)\right)^{-1} \cap\left\{t<t_{2}\right\}\right)$, we set

$$
u_{\varphi}(z)=\int_{\mathbb{R}^{N+1}} \Gamma(z, \zeta) \varphi(\zeta) d \zeta, \quad z \in \mathbb{R}^{N+1} .
$$

Hence, we obtain $u_{\varphi} \in C^{\infty}\left(\mathbb{R}^{N+1}\right), u_{\varphi} \geq 0$ and $\mathscr{L} u_{\varphi}=-\varphi \leq 0$, so that, by Remark 5.3, $u_{\varphi} \in \overline{\mathcal{S}}\left(\mathbb{R}^{N+1}\right)$. Moreover the choice of $\varphi$ implies that $u_{\varphi}\left(z_{1}\right)=0$ and $u_{\varphi}\left(z_{2}\right)>0$.

In the case $t_{1}=t_{2}, x_{1} \neq x_{2}$, we consider the sequence

$$
\mathcal{O}_{n}\left(z_{2}\right)=\left\{\zeta \in \mathbb{R}^{N+1}: \Gamma\left(z_{2}, \zeta\right)>n^{Q-2}\right\}, \quad n \in \mathbb{N} .
$$

We note that $\mathcal{O}_{n}\left(z_{2}\right)$ shrinks to $\left\{z_{2}\right\}$ as $n \rightarrow \infty$, by property viii) of the fundamental solution. For any $\varphi_{n} \in C_{0}^{\infty}\left(\mathcal{O}_{n}\left(z_{2}\right)\right)$ such that $\int \varphi_{n}=1$ and $\varphi_{n} \geq 0$, we define $u_{\varphi_{n}}$ as in (5.14). Then, $u_{\varphi_{n}}$ is a smooth non-negative function in $\mathbb{R}^{N+1}$ satisfying $\mathscr{L} u_{\varphi_{n}} \leq 0$, and so $u_{\varphi_{n}}$ is $\mathscr{L}$ superharmonic. It holds

$$
\begin{gathered}
u_{\varphi_{n}}\left(z_{2}\right)=\int_{\mathbb{R}^{N+1}} \Gamma\left(z_{2}, \zeta\right) \varphi_{n}(\zeta) d \zeta \geq n^{Q-2} \quad \text { for every } n \in \mathbb{N} ; \\
u_{\varphi_{n}}\left(z_{1}\right) \leq \max _{\zeta \in \overline{\mathcal{O}_{1}\left(z_{2}\right)}} \Gamma\left(z_{1}, \zeta\right)=C,
\end{gathered}
$$

where $C$ is a real positive constant independent of $n$. This ends the proof.

We summarize the above facts in the following

Proposition 5.6 Let $\mathscr{L}$ be an operator in the form (5.1) and assume that $[\mathbf{H}]$ and $[\mathbf{L}]$ are satisfied. The map $\mathcal{H}$ which associates any open set $\mathcal{O} \subseteq \mathbb{R}^{N+1}$ with the linear space of the $\mathscr{L}$-harmonic functions in $\mathcal{O}$ is a harmonic sheaf, and $\left(\mathbb{R}^{N+1}, \mathcal{H}\right)$ is a $\mathfrak{B}$-harmonic space.

A remarkable feature of a $\mathfrak{B}$-harmonic space is that the Wiener resolutivity theorem holds (see $[4,18])$. In order to state it, we introduce some additional notations. We recall that if $\mathcal{O} \subset \mathbb{R}^{N+1}$ is a bounded open set, then an extended real function $f: \partial \mathcal{O} \rightarrow[-\infty, \infty]$ is called resolutive if

$$
\inf \overline{\mathcal{U}}_{f}^{\mathcal{O}}=\sup \underline{\mathcal{U}}_{f}^{\mathcal{O}}=: H_{f}^{\mathcal{O}} \in \mathcal{H}(\mathcal{O}),
$$

where

$$
\begin{aligned}
& \overline{\mathcal{U}}_{f}^{\mathcal{O}}=\left\{u \in \overline{\mathcal{S}}(\mathcal{O}): \inf _{\mathcal{O}} u>-\infty \text { and } \liminf _{z \rightarrow \zeta} u(z) \geq f(\zeta), \forall \zeta \in \partial \mathcal{O}\right\}, \\
& \underline{\mathcal{U}}_{f}^{\mathcal{O}}=\left\{u \in \underline{\mathcal{S}}(\mathcal{O}): \sup _{\mathcal{O}} u<\infty \text { and } \limsup _{z \rightarrow \zeta} u(z) \leq f(\zeta), \forall \zeta \in \partial \mathcal{O}\right\} .
\end{aligned}
$$


We say that $H_{f}^{\mathcal{O}}$ is the generalized solution in the sense of Perron-Wiener-Brelot to the problem

$$
u \in \mathcal{H}(\mathcal{O}), \quad u=f \quad \text { on } \partial \mathcal{O} .
$$

The Wiener resolutivity theorem yields that any $f \in C(\partial \mathcal{O}, \mathbb{R})$ is resolutive. The map $C(\partial \mathcal{O}, \mathbb{R}) \ni f \mapsto H_{f}^{\mathcal{O}}(z)$ defines a linear positive functional for every $z \in \mathcal{O}$. Again, there exists a Radon measure $\mu_{z}^{\mathcal{O}}$ on $\partial \mathcal{O}$ such that

$$
H_{f}^{\mathcal{O}}(z)=\int_{\partial \mathcal{O}} f(\zeta) \mathrm{d} \mu_{z}^{\mathcal{O}}(\zeta)
$$

We call $\mu_{z}^{\mathcal{O}}$ the $\mathscr{L}$-harmonic measure relative to $\mathcal{O}$ and $z$, and when $\mathcal{O}$ is $\mathscr{L}$-regular this definition coincides with the one in (5.12). Finally, a point $\zeta \in \partial \mathcal{O}$ is called $\mathscr{L}$-regular for $\mathcal{O}$ if

$$
\lim _{\mathcal{O} \ni z \rightarrow \zeta} H_{f}^{\mathcal{O}}(z)=f(\zeta), \quad \text { for every } f \in C(\partial \mathcal{O}, \mathbb{R}) .
$$

Obviously, $\mathcal{O}$ is $\mathscr{L}$-regular if and only if every $\zeta \in \partial \mathcal{O}$ is $\mathscr{L}$-regular.

\subsection{Harnack inequalities}

Let $\mathcal{O} \subset \mathbb{R}^{N+1}$ be an open set. A closed subset $F$ of $\mathcal{O}$ is called an absorbent set if, for any $z \in F$ and any $\mathscr{L}$-regular neighborhood $V \subset \bar{V} \subset \mathcal{O}$ of $z$, it holds $\mu_{z}^{V}(\partial V \backslash F)=0$. For any given $z_{0} \in \mathcal{O}$ we set

$$
\mathscr{F}_{z_{0}}=\left\{F \subset \mathcal{O}: F \ni z_{0}, F \text { is an absorbent set }\right\}
$$

Then,

$$
\mathcal{O}_{z_{0}}=\bigcap_{F \in \mathscr{F}_{z_{0}}} F
$$

is the smallest absorbent set containing $z_{0}$. The Potential Theory provides us with the following Harnack inequality. Let $\left(\mathbb{R}^{N+1}, \mathcal{H}\right)$ be a $\mathfrak{B}$-harmonic space, let $\mathcal{O}$ be an open subset of $\mathbb{R}^{N+1}$ and let $z_{0} \in \mathcal{O}$. Then,

$$
\text { for every compact set } K \subset \operatorname{Int}\left(\mathcal{O}_{z_{0}}\right), \sup _{K} u \leq C_{K} u\left(z_{0}\right) \text {, }
$$

for any non-negative function $u \in \mathcal{H}(\mathcal{O})$. Here $C_{K}$ is a positive constant depending on $\mathcal{O}, K, z_{0}$. We refer to Theorem 1.4.4 in [4] and Proposition 6.1.5 in [18]. Proposition 5.6 implies that (5.19) applies to our operator $\mathscr{L}$. We summarize the above argument in the following

Proposition 5.7 Let $\mathscr{L}$ be an operator in the form (5.1) satisfying $[\mathrm{H}]$ and $[\mathrm{L}]$, let $\mathcal{O} \subseteq$ $\mathbb{R}^{N+1}$ be an open set, and let $z_{0} \in \mathcal{O}$. Then,

$$
\text { for every compact set } K \subset \operatorname{Int}\left(\mathcal{O}_{z_{0}}\right), \sup _{K} u \leq C_{K} u\left(z_{0}\right) \text {, }
$$

for any non-negative solutions $u$ to $\mathscr{L} u=0$ in $\mathcal{O}$. Here $C_{K}$ is a positive constant depending on $\mathcal{O}, K, z_{0}$ and on $\mathscr{L}$. 
In order to prove Theorem 5.2 we give the following

Lemma 5.8 Let $\mathscr{L}$ be an operator as in (5.1) satisfying $[\mathrm{H}]$ and $[\mathrm{L}]$, and let $\mathcal{O}$ be an open subset of $\mathbb{R}^{N+1}$. For any given $z_{0} \in \mathcal{O}$, we have $\mathscr{A}_{z_{0}} \subseteq \mathcal{O}_{z_{0}}$ with $\mathscr{A}_{z_{0}}$ defined in (5.6).

Proof. Since $\mathcal{O}_{z_{0}}$ is a closed set, and $\mathscr{A}_{z_{0}}$ is the closure of the set $A_{z_{0}}$ defined in (5.6), it is sufficient to show that $A_{z_{0}} \subseteq \mathcal{O}_{z_{0}}$. By contradiction, assume that $\bar{z} \in A_{z_{0}} \backslash \mathcal{O}_{z_{0}}$. Then, there exists an $\mathscr{L}$-admissible path $\gamma:[0, T] \rightarrow \mathcal{O}$ such that $\gamma(0)=z_{0}, \gamma(T)=\bar{z}$.

We set

$$
\left.\left.t_{1}:=\inf \{t>0: \gamma(] t, T]\right) \cap \mathcal{O}_{z_{0}}=\emptyset\right\} .
$$

Note that, since $\mathcal{O} \backslash \mathcal{O}_{z_{0}}$ is an open set containing $\bar{z}$ and $\gamma$ is a continuous curve, there exists an open neighborhood $U \subseteq \mathcal{O}$ of $\bar{z}$ such that $U \cap \mathcal{O}_{z_{0}}=\emptyset$, and a positive $\sigma$ satisfying $\gamma(] T-\sigma, T]) \subseteq U$. Hence, $t_{1} \in\left[0, T\left[\right.\right.$ is well defined and we have $\gamma(t) \notin \mathcal{O}_{z_{0}}$ for every $\left.\left.t \in\right] t_{1}, T\right]$. Again, by the continuity of $\gamma$, we have

$$
z_{1}=\gamma\left(t_{1}\right) \in \mathcal{O}_{z_{0}} .
$$

Let $V \subset \bar{V} \subset \mathcal{O}$ be a $\mathscr{L}$-regular neighborhood of $z_{1}$ with $\bar{z} \notin \bar{V}$. Arguing as above, we can find $\left.t_{2} \in\right] t_{1}, T\left[\right.$ such that $\gamma\left(\left[t_{1}, t_{2}[) \subset V\right.\right.$ and $z_{2}=\gamma\left(t_{2}\right) \in \partial V$. Consider any neighborhood $W$ of $z_{2}$, such that $W \subset \mathcal{O} \backslash \mathcal{O}_{z_{0}}$. Let $\varphi \in C(\partial V)$ be any non-negative function, supported in $W \cap \partial V$, and such that $\varphi\left(z_{2}\right)>0$. Recalling that the harmonic function $H_{\varphi}^{V}$ is non-negative, we aim to show that

$$
H_{\varphi}^{V}\left(z_{1}\right)>0
$$

By contradiction, we suppose that $H_{\varphi}^{V}$ vanishes at $z_{1}$. In other terms, $H_{\varphi}^{V}$ attains its minimum value at $z_{1}$, then Bony's minimum principle implies $H_{\varphi}^{V} \equiv 0$ in $\gamma\left(\left[t_{1}, t_{2}[)\right.\right.$. As a consequence, since $H_{\varphi}^{V}$ satisfies (5.11),

$$
\lim _{t \rightarrow t_{2}^{-}} H_{\varphi}^{V}(\gamma(t))=0
$$

On the other hand, by the choice of $\varphi$

$$
\lim _{V \ni z \rightarrow z_{2}} H_{\varphi}^{V}(z)=\varphi\left(z_{2}\right)>0 .
$$

This contradicts (5.21) and proves (5.20). By using representation (5.12) of $H_{\varphi}^{V}$ in terms of the $\mathscr{L}$-harmonic measure, (5.20) reads as follows

$$
H_{\varphi}^{V}\left(z_{1}\right)=\int_{\partial V \cap W} \varphi(\zeta) d \mu_{z_{1}}^{V}(\zeta)>0, \quad \text { then } \quad \mu_{z_{1}}^{V}(\partial V \cap W)>0 .
$$

On the other hand, $z_{1}$ belongs to the absorbent set $\mathcal{O}_{z_{0}}$, so that $\mu_{z_{1}}^{V}\left(\partial V \backslash \mathcal{O}_{z_{0}}\right)=0$. But this clashes with (5.22), being $W \subseteq \mathcal{O} \backslash \mathcal{O}_{z_{0}}$. This accomplishes the proof.

Proof of Theorem 5.2. It is a plain consequence of Proposition 5.7 and Lemma 5.8

As the following proposition shows, we are able to give a complete characterization of the set $\mathcal{O}_{z_{0}}$ if $\mathscr{A}_{z_{0}}$ is an absorbent set as well. 
Proposition 5.9 Let $\mathscr{L}$ be an operator as in (5.1) satisfying $[\mathrm{H}]$ and $[\mathrm{L}]$, let $\mathcal{O} \subseteq \mathbb{R}^{N+1}$ be an open set, and let $z_{0} \in \mathcal{O}$. If $\mathscr{A}_{z_{0}}$ is an absorbent set, then $\mathscr{A}_{z_{0}} \equiv \mathcal{O}_{z_{0}}$.

Proof. The claim directly follows from Lemma 5.8, recalling the definition of $\mathcal{O}_{z_{0}}$.

The first statement in next proposition is a classical result in abstract potential theory (see e.g. [4, Theorem 1.4.1] and [18, Proposition 6.1.1]). For the convenience of the reader, we explicitly give here its simple proof.

Proposition 5.10 Let $\mathscr{L}$ be an operator as in (5.1) satisfying $[\mathrm{H}]$ and $[\mathrm{L}]$, let $\mathcal{O} \subseteq \mathbb{R}^{N+1}$ be an open set, and let $z_{0} \in \mathcal{O}$. Assume that there exists a solution $u \geq 0$ to $\mathscr{L} u=0$ in $\mathcal{O}$ such that $u \equiv 0$ in $\mathscr{A}_{z_{0}}$ and $u>0$ in $\mathcal{O} \backslash \mathscr{A}_{z_{0}}$. Then $\mathscr{A}_{z_{0}}$ is an absorbent set, and $\mathscr{A}_{z_{0}} \equiv \mathcal{O}_{z_{0}}$.

Proof. Since $u$ is continuous and non-negative,

$$
\mathscr{A}_{z_{0}}=\{z \in \mathcal{O}: u(z) \leq 0\}
$$

is a closed subset of $\mathcal{O}$. Let $z \in \mathscr{A}_{z_{0}}$, and let $V \subset \bar{V} \subset \mathcal{O}$ be a $\mathscr{L}$-regular neighborhood of $z$. As $u \in \mathcal{H}(\mathcal{O})$, we have

$$
0 \geq u(z)=\int_{\partial V} u(\zeta) d \mu_{z}^{V}(\zeta) \geq 0, \quad \text { so that } \quad \mu_{z}^{V}\left(\partial V \backslash \mathscr{A}_{z_{0}}\right)=0 .
$$

Hence $\mathscr{A}_{z_{0}}$ is an absorbent set. The last statement plainly follows from Proposition 5.9.

\subsection{Lifting and Harnack inequalities}

We first consider the $\operatorname{PDE}$ (5.8) for $k=2$. Note that, in this case, it is equivalent to (5.9), and reads as follows

$$
\mathscr{L}=\frac{1}{2} \Delta_{x_{1, n}}+\left|x_{1, n}\right|^{2} \partial_{x_{n+1}}-\partial_{t} .
$$

It is homogeneous with respect to the following dilation

$$
\delta_{\lambda}(x, t)=\left(\lambda x_{1, n}, \lambda^{4} x_{n+1}, \lambda^{2} t\right) .
$$

Even if $\mathscr{L}$ does not satisfy $[\mathbf{L}]-i)$, it has a fundamental solution $\Gamma$ which shares several properties of the usual heat kernels. We remark that, since $\mathscr{L}$ does not satisfy the controllability condition $[\mathbf{C}]$, the support of $\Gamma$ is strictly contained in the half space $\{t<\tau\}$.

We next show that $\mathscr{L}$ can be lifted to a suitable operator $\widetilde{\mathscr{L}}$ in the form (5.1) satisfying both $[\mathbf{H}]$ and $[\mathbf{L}]$. By adding a new variable $y=y_{1, n} \in \mathbb{R}^{n}$, we define the following vector fields on $\mathbb{R}^{2 n+2}$

$$
\widetilde{Y}_{i}=Y_{i}=\partial_{x_{i}}, i \in \llbracket 1, n \rrbracket, \quad \widetilde{Z}=\left|x_{1, n}\right|^{2} \partial_{x_{n+1}}+\sum_{i=1}^{n} x_{i} \partial_{y_{i}}-\partial_{t} .
$$

Clearly, if we denote $v(x, y, t)=u(x, t)$ for any $u \in C^{\infty}\left(\mathbb{R}^{n+2}\right)$, we have

$$
\widetilde{Y}_{i} v(x, y, t)=Y_{i} u(x, t), \quad \forall i \in \llbracket 1, n \rrbracket, \quad \widetilde{Z} v(x, y, t)=Z u(x, t),
$$


then, if we consider the lifted operator $\widetilde{\mathscr{L}}=\frac{1}{2} \sum_{i=1}^{n} \widetilde{Y}_{i}^{2}+\widetilde{Z}$, we find $\widetilde{\mathscr{L}} v(x, y, t)=\mathscr{L} u(x, t)$. By a standard procedure (see e.g., [7, Chapter 1]), we explicitly write the group law $\circ$ of the homogeneous Lie group $\mathbb{G}=\left(\mathbb{R}^{2 n+2}, \circ,\left(\widetilde{\delta}_{\lambda}\right)_{\lambda>0}\right)$ such that $\widetilde{\mathscr{L}}$ is $\mathbb{G}$-Lie-invariant:

$(x, y, t) \circ(\xi, \eta, \tau)=\left(x_{1, n}+\xi_{1, n}, x_{n+1}+\xi_{n+1}+2\left\langle x_{1, n}, \eta_{1, n}\right\rangle-\tau\left|x_{1, n}\right|^{2}, y_{1, n}+\eta_{1, n}-\tau x_{1, n}, t+\tau\right)$,

and the dilation $\widetilde{\delta}_{\lambda}$ :

$$
\widetilde{\delta}_{\lambda}(x, y, t)=\left(\lambda x_{1, n}, \lambda^{4} x_{n+1}, \lambda^{3} y_{1, n}, \lambda^{2} t\right) .
$$

Therefore, the lifted operator $\widetilde{\mathscr{L}}$ satisfies $[\mathbf{H}]$ and $[\mathbf{L}]$. In the sequel we will consider admissible paths in the following form

$$
\widetilde{\gamma}^{\prime}(s)=\sum_{j=1}^{n} \omega_{j} \widetilde{Y}_{j}(\widetilde{\gamma}(s))+\widetilde{Z}(\widetilde{\gamma}(s)), \quad s \in[0, \widetilde{\tau}]
$$

for some constant vector $\omega=\left(\omega_{1}, \ldots, \omega_{n}\right), \widetilde{\gamma}(0)=(x, y, t)$. Its explicit expression is

$$
\widetilde{\gamma}(s)=\left(x_{1, n}+s \omega, x_{n+1}+s\left|x_{1, n}\right|^{2}+s^{2}\left\langle x_{1, n}, \omega\right\rangle+\frac{s^{3}}{3}|\omega|^{2}, y+s x_{1, n}+\frac{s^{2}}{2} \omega, t-s\right) .
$$

In order to prove an invariant Harnack inequality for the non-negative solutions to $\widetilde{\mathscr{L} v}=$ 0 , we describe the sets $\mathcal{O}_{z_{0}}$ and $\mathscr{A}_{z_{0}}$ in the case when $z_{0}$ is the origin and

$$
\mathcal{O}=\left\{(x, y, t) \in \mathbb{R}^{2 n+2}|| x_{1, n}\left|<1,-1<x_{n+1}<1,\right| y \mid<1,-1<t<1\right\} .
$$

Lemma 5.11 Let $\mathcal{O}$ be the open set defined in (5.29), and let $z_{0}=(0,0,0)$. Then

$$
\mathscr{A}_{z_{0}}=\left\{\left.(x, y, t) \in \mathcal{O}\left|0 \leq x_{n+1} \leq-t,\right| y\right|^{2} \leq-t x_{n+1}\right\}
$$

and $\mathcal{O}_{z_{0}}=\mathscr{A}_{z_{0}}$.

Proof. In order to prove (5.30), we consider any $\widetilde{\mathscr{L}}$-admissible curve $\gamma$ in $\mathcal{O}$. In our setting, the components $x_{n+1}, y_{1, n}$ and $t$ of every diffusion trajectory are constant functions. Moreover, any drift trajectory $\gamma:[0, T] \rightarrow \mathcal{O}$ starting from $(\bar{x}, \bar{y}, \bar{t})$ is given by

$$
\gamma(s)=\left(\bar{x}_{1, n}, \bar{x}_{n+1}+s\left|\bar{x}_{1, n}\right|^{2}, \bar{y}+s \bar{x}_{1, n}, \bar{t}-s\right) .
$$

Hence, any $\widetilde{\mathscr{L}}$-admissible curve $\gamma:[0, T] \rightarrow \mathcal{O}$ with $\gamma(0)=(0,0,0)$ is given by

$$
\gamma(s)=\left(x_{1, n}(s), \int_{0}^{s} \sum_{k=1}^{m}\left|c_{k}\right|^{2} \mathbb{I}_{I_{k}}(r) d r, \int_{0}^{s} \sum_{k=1}^{m} c_{k} \mathbb{I}_{I_{k}}(r) d r,-\int_{0}^{s} \sum_{k=1}^{m} \mathbb{I}_{I_{k}}(r) d r\right), \quad s \in[0, T] .
$$

Here $I_{1}, \ldots, I_{m}$ are disjoint intervals contained in $[0, T]$ and $\mathbb{I}_{I_{k}}$ denotes the characteristic function of $I_{k}$. The function $x_{1, n}$ is constant on every $I_{k}$, and any $c_{k}$ is a constant vector such that $\left|c_{k}\right| \leq 1$ for $k=1, \ldots, m$. As a consequence of the Hölder inequality we find

$$
\mathscr{A}_{z_{0}} \subseteq\left\{\left.(x, y, t) \in \mathcal{O}\left|0 \leq x_{n+1} \leq-t,\right| y\right|^{2} \leq-t x_{n+1}\right\} .
$$


In order to prove the opposite inclusion, we consider any point

$$
(\bar{x}, \bar{y}, \bar{t}) \in\left\{\left.(x, y, t) \in \mathcal{O}\left|0<x_{n+1}<-t,\right| y\right|^{2}<-t x_{n+1}\right\}, \quad \bar{y} \neq 0,
$$

and we show that there exists a $\widetilde{\mathscr{L}}$-admissible curve $\gamma=\gamma_{1}+\gamma_{2}+\cdots+\gamma_{5}$ contained in $\mathcal{O}$, which steers $(0,0,0)$ to $(\bar{x}, \bar{y}, \bar{t})$. To this aim, we fix a small positive $\varepsilon$, that will be specified in the sequel, and we set

$$
s_{\varepsilon}=\frac{-\bar{t} \bar{x}_{n+1}-|\bar{y}|^{2}}{\bar{x}_{n+1}-2|\bar{y}|(1-\varepsilon)-\bar{t}(1-\varepsilon)^{2}} .
$$

Note that $-\bar{t} \bar{x}_{n+1}+2|\bar{y}| \bar{t}(1-\varepsilon)+\bar{t}^{2}(1-\varepsilon)^{2} \geq(|\bar{y}|+\bar{t}(1-\varepsilon))^{2}$, so that $0<s_{\varepsilon}<-\bar{t}$. We set $\widetilde{x}_{1, n}=\frac{1-\varepsilon}{|\bar{y}|} \bar{y}$ and we choose $\gamma_{1}$ as a diffusion trajectory connecting $(0,0,0)$ to $\left(\widetilde{x}_{1, n}, 0,0,0\right)$, and $\gamma_{2}:\left[0, s_{\varepsilon}\right] \rightarrow \mathbb{R}^{2 n+2}$ as a drift trajectory starting from $\left(\widetilde{x}_{1, n}, 0,0,0\right)$. Hence, according to $(5.31)$, we find $\gamma_{2}\left(s_{\varepsilon}\right)=\left(\widetilde{x}_{1, n}, s_{\varepsilon}(1-\varepsilon)^{2}, s_{\varepsilon} \frac{1-\varepsilon}{|\bar{y}|} \bar{y},-s_{\varepsilon}\right)$. Then, by a diffusion trajectory $\gamma_{3}$, we connect $\gamma_{2}\left(s_{\varepsilon}\right)$ to the point $\left(\frac{|\bar{y}|-s_{\varepsilon}(1-\varepsilon)}{\left(-\bar{t}-s_{\varepsilon}\right)|\bar{y}|} \bar{y}, s_{\varepsilon}(1-\varepsilon)^{2}, s_{\varepsilon} \frac{1-\varepsilon}{|\bar{y}|} \bar{y},-s_{\varepsilon}\right)$. We next consider a drift path $\gamma_{4}:\left[0,-\bar{t}-s_{\varepsilon}\right] \rightarrow \mathbb{R}^{2 n+2}$ which, by (5.31), and by our choice of $s_{\varepsilon}$, steers the end point of $\gamma_{3}$ to $\left(\frac{|\bar{y}|-s_{\varepsilon}(1-\varepsilon)}{\left(-\bar{t}-s_{\varepsilon}\right)|\bar{y}|} \bar{y}, \bar{x}_{n+1}, \bar{y}, \bar{t}\right)$. Finally, we can find a diffusion path $\gamma_{5}$ connecting $\gamma_{4}\left(-\bar{t}-s_{\varepsilon}\right)$ to $(\bar{x}, \bar{y}, \bar{t})$.

Clearly, $\gamma=\gamma_{1}+\gamma_{2}+\cdots+\gamma_{5}$ is a $\widetilde{\mathscr{L}}$-admissible curve of $\mathbb{R}^{2 n+2}$ connecting $(0,0,0)$ to $(\bar{x}, \bar{y}, \bar{t})$. Next we prove that, for sufficiently small $\varepsilon$, the trajectory $\gamma$ is contained in $\mathcal{O}$. To this aim, as the set $\mathcal{O}$ is convex and the paths $\gamma_{1}, \gamma_{2}, \ldots, \gamma_{5}$ are segments, we only need to show that the end-points of $\gamma_{1}, \gamma_{2}, \gamma_{3}, \gamma_{4}$ belong to $\mathcal{O}$. The inequalities $-1<\frac{|\bar{y}|-s_{\varepsilon}(1-\varepsilon)}{-\bar{t}-s_{\varepsilon}}<1$ directly follow from the definition of $s_{\varepsilon}$, for sufficiently small positive $\varepsilon$. The other inequalities are a plain consequence of the fact that $0<s_{\varepsilon}<-\bar{t}<1$, as previously noticed. Since $\mathscr{A}_{z_{0}}$ is the closure of the set of the points that can be reached by a $\widetilde{\mathscr{L}}$-admissible path, we get

$$
\left\{\left.(x, y, t) \in \mathcal{O}\left|0 \leq x_{n+1} \leq-t,\right| y\right|^{2} \leq-t x_{n+1}\right\} \subseteq \mathscr{A}_{z_{0}} .
$$

This concludes the proof of (5.30).

To complete the proof, by Proposition 5.10 it is sufficient to find a non-negative solution $v$ of $\widetilde{\mathscr{L}} v=0$, such that $v \equiv 0$ in $\mathscr{A}_{z_{0}}$, and $v>0$ in $\mathcal{O} \backslash \mathscr{A}_{z_{0}}$. Let $\varphi$ be any function in $C(\partial \mathcal{O})$, such that $\varphi \equiv 0$ in $\partial \mathcal{O} \cap \mathscr{A}_{z_{0}}$ and $\varphi>0$ in $\partial \mathcal{O} \backslash \mathscr{A}_{z_{0}}$. Then the Perron-Wiener-Brelot solution $v:=H_{\varphi}^{\mathcal{O}}$ of the following Cauchy-Dirichlet problem

$$
\begin{cases}\widetilde{\mathscr{L}} v=0 & \text { in } \mathcal{O} \\ v=\varphi & \text { in } \partial \mathcal{O}\end{cases}
$$

is non-negative. Next we prove that $v>0$ in $\mathcal{O} \backslash \mathscr{A}_{z_{0}}$. By contradiction, let $(x, y, t) \in \mathcal{O} \backslash \mathscr{A}_{z_{0}}$ be such that $v(x, y, t)=0$. Then $(x, y, t)$ is a minimum for $v$, so that from Bony's minimum principle [9, Théorème 3.2] it follows that $v\left(\widetilde{x}_{1, n}, x_{n+1}, y, t\right)=\varphi\left(\widetilde{x}_{1, n}, x_{n+1}, y, t\right)=0$, for every $\widetilde{x}_{1, n} \in \partial(]-1,1\left[^{n}\right)$. Since every point $\left(\widetilde{x}_{1, n}, x_{n+1}, y, t\right)$ is regular for the Dirichlet problem, and belongs to $\partial \mathcal{O} \backslash \mathscr{A}_{z_{0}}$, we find a contradiction with our assumption on $\varphi$. Suppose now that there exists $(x, y, t) \in \mathscr{A}_{z_{0}}$ such that $v(x, y, t)>0$. Since every point of the set 
$\partial \mathcal{O} \cap \mathscr{A}_{z_{0}}$ is $\widetilde{\mathscr{L}}$-regular, $v$ is continuous in $\mathscr{A}_{z_{0}}$. Hence there exists a $(\bar{x}, \bar{y}, \bar{t}) \in \mathscr{A}_{z_{0}}$ such that $v(\bar{x}, \bar{y}, \bar{t})=\max _{\mathscr{A}_{z_{0}}} v>0$. By Bony's minimum principle we have $v\left(\widetilde{x}_{1, n}, \bar{x}_{n+1}, \bar{y}, \bar{t}\right)=$ $\varphi\left(\widetilde{x}_{1, n}, \bar{x}_{n+1}, \bar{y}, \bar{t}\right)>0$, for any $\widetilde{x}_{1, n} \in \partial(]-1,1\left[{ }^{n}\right)$, and this fact contradicts our assumption on $\varphi$.

Next we introduce some notations to state a Harnack inequality which is invariant with respect to the group law o defined in (5.26) and the dilation $\widetilde{\delta}_{r}$ introduced in (5.27). Consider the box $\left.\left.Q_{r}=\right]-r, r\left[{ }^{n} \times\right]-r^{4}, r^{4}[\times]-r^{3}, r^{3}\left[{ }^{n} \times\right]-r^{2}, 0\right]$, and note that $Q_{r}=\widetilde{\delta}_{r} Q_{1}$. For every compact set $\mathcal{K} \subseteq Q_{1}$, for any positive $r$ and for any $z_{0} \in \mathbb{R}^{2 n+2}$ we denote by

$$
Q_{r}\left(z_{0}\right)=z_{0} \circ \widetilde{\delta}_{r} Q_{1}=\left\{z_{0} \circ \widetilde{\delta}_{r} \zeta \mid \zeta \in Q_{1}\right\}, \quad \mathcal{K}_{r}\left(z_{0}\right)=z_{0} \circ \widetilde{\delta}_{r} \mathcal{K} .
$$

Corollary 5.12 For every compact set $\mathcal{K} \subseteq\left\{\left.(x, y, t) \in Q_{1}\left|0<x_{n+1}<-t,\right| y\right|^{2}<-t x_{n+1}\right\}$, $r>0$ and $z_{0} \in \mathbb{R}^{2 n+2}$ there exists a positive constant $C_{\mathcal{K}}$, depending only on $\widetilde{\mathscr{L}}$ and $\mathcal{K}$, such that

$$
\sup _{\mathcal{K}_{r}\left(z_{0}\right)} v \leq C_{\mathcal{K}} v\left(z_{0}\right)
$$

for every non-negative solution $v$ of $\widetilde{\mathscr{L} v}=0$ on any open set containing $\bar{Q}_{r}\left(z_{0}\right)$.

Proof. Consider the function $w(z)=v\left(z_{0} \circ \widetilde{\delta}_{r} z\right)$. By the invariance with respect to $\widetilde{\delta}_{r}$ and $\circ$, we have $\widetilde{\mathscr{L}} w=0$ in $Q_{1}$. Aiming to apply Theorem 5.2, we consider the open set $\mathcal{O}$ defined in (5.29), and we note that $\mathcal{O} \cap\{t<0\} \subset Q_{1}$. Then $w$ is defined as a continuous function on $\partial \mathcal{O} \cap\{t<0\}$. We extend $w$ to a continuous function on $\partial \mathcal{O}$, and we solve the boundary value problem $\widetilde{\mathscr{L}} \widetilde{w}=0$ in $\widetilde{Q}_{1}$, with $\widetilde{w}=w$ in $\partial \mathcal{O}$. Then we apply Theorem 5.2 and Lemma 5.11 , and we get $\sup _{\mathcal{K}} \widetilde{w} \leq C_{\mathcal{K}} \widetilde{w}(0,0,0)$. By the comparison principle we have $\widetilde{w}=w$ in $\mathcal{O} \cap\{t \leq 0\}$, then the claim plainly follows from the inclusion $\mathcal{K} \subset \mathcal{O} \cap\{t<0\}$.

We are now ready to build a Harnack chain for (5.23) by using the following set

$$
\mathcal{K}=\left\{(x, y, t) \in \mathbb{R}^{2 n+2}|| x_{1, n}\left|\leq \frac{1}{2}, \frac{1}{32} \leq x_{n+1} \leq \frac{1}{4},\right| y \mid \leq \frac{1}{8}, t=-\frac{1}{2}\right\}
$$

which is a compact subset of $\left\{\left.(x, y, t) \in Q_{1}\left|0<x_{n+1}<-t,\right| y\right|^{2}<-t x_{n+1}\right\}$. Before doing that for $k=2$ only, we extend the above procedure to equations (5.8) and (5.9) for $k>2$.

We next show that, in both cases (5.8) and (5.9), $\mathscr{L}$ can be lifted to a suitable operator $\widetilde{\mathscr{L}}$ in the form (5.1) satisfying $[\mathbf{H}]$ and $[\mathbf{L}]$. We introduce a new variable $y \in \mathbb{R}^{(k-1) n}$, that will be denoted as follows $y=\left(y_{1}, y_{2}, \ldots, y_{(k-1)}\right)$, with $y_{j}=\left(y_{j 1}, \ldots, y_{j n}\right) \in \mathbb{R}^{n}$ for $j \in \llbracket 1, k-1 \rrbracket$. We then define the lifted vector fields on $\mathbb{R}^{k n+2}$ :

$$
\widetilde{Y}_{i}=Y_{i}=\partial_{x_{i}}, \quad i \in \llbracket 1, n \rrbracket, \quad \widetilde{Z}=Z+\sum_{i=1}^{k-1} \sum_{j=1}^{n} x_{j}^{i} \partial_{y_{i j}} ;
$$

where $Z=\left|x_{1, n}\right|^{k} \partial_{x_{n+1}}-\partial_{t}$ for (5.8), and $Z=\sum_{j=1}^{n} x_{j}^{k} \partial_{x_{n+1}}-\partial_{t}$ for (5.9). If we denote $v(x, y, t)=u(x, t)$ for any $u \in C^{\infty}\left(\mathbb{R}^{n+2}\right)$, we have

$$
\widetilde{Y}_{i} v(x, y, t)=Y_{i} u(x, t), \quad \forall i \in \llbracket 1, n \rrbracket, \quad \widetilde{Z} v(x, y, t)=Z u(x, t) .
$$


Then, setting $\widetilde{\mathscr{L}}=\frac{1}{2} \sum_{i=1}^{n} \widetilde{Y}_{i}^{2}+\widetilde{Z}$, we plainly find $\widetilde{\mathscr{L}} v(x, y, t)=\mathscr{L} u(x, t)$.

Since $\operatorname{dim}\left(\operatorname{Lie}\left\{\tilde{Y}_{1}, \ldots, \widetilde{Y}_{n}, \widetilde{Z}\right\}\right)=k n+2$ and $\operatorname{rank}\left(\operatorname{Lie}\left\{\widetilde{Y}_{1}, \ldots, \widetilde{Y}_{n}, \widetilde{Z}\right\}(x, y, t)\right)=k n+2$ at every point $(x, y, t) \in \mathbb{R}^{k n+2}$, Theorem 1.1 in [6] yields the existence of a homogeneous Lie group $\mathbb{G}=\left(\mathbb{R}^{k n+2}, \circ,\left(\widetilde{\delta}_{\lambda}\right)_{\lambda>0}\right)$ such that $\widetilde{\mathscr{L}}$ is Lie-invariant on $\mathbb{G}$. Therefore, the lifted operators $\widetilde{\mathscr{L}}$ satisfy $[\mathbf{H}]$ and $[\mathbf{L}]$. The dilation $\widetilde{\delta}_{\lambda}$ acts as follows:

$$
\widetilde{\delta}_{\lambda}(x, y, t)=\left(\lambda x_{1, n}, \lambda^{k+2} x_{n+1}, \lambda^{3} y_{1}, \ldots, \lambda^{k+1} y_{k-1}, \lambda^{2} t\right),
$$

for every $(x, y, t) \in \mathbb{R}^{k n+2}$, and $\lambda>0$. We next aim to apply Theorem 5.2 in order to prove a Harnack inequality on the lifted space $\mathbb{R}^{k n+2}$. For any $\omega \in L^{2}\left([-T, T], \mathbb{R}^{n}\right)$ for every $(x, y, t) \in \mathbb{R}^{k n+2}$ and $T>0$, we denote by $\widetilde{\gamma}:[-T, T] \rightarrow \mathbb{R}^{k n+2}$ the solution of the Cauchy problem

$$
\left\{\begin{array}{l}
\widetilde{\gamma}^{\prime}(s)=\sum_{j=1}^{n} \omega_{j}(s) \widetilde{Y}_{j}(\widetilde{\gamma}(s))+\widetilde{Z}(\widetilde{\gamma}(s)), \quad s \in[-T, T], \\
\widetilde{\gamma}(0)=(x, y, t) .
\end{array}\right.
$$

In order to simplify the notation, in the sequel we will denote the solution of (5.36) as

$$
\gamma(s)=\left(x_{1, n}(s), x_{n+1}(s), y(s), t(s)\right), \quad s \in[-T, T] .
$$

Note that $t(s)=t-s$ for every $s \in[-T, T]$, so that $t(T)=t-T$.

The composition law "o" of $G$ is related to (5.36) as follows: if $(\bar{x}, \bar{y}, \bar{t})=\widetilde{\gamma}(T)$ is the end point of the path $\widetilde{\gamma}$ defined by (5.36) with $\widetilde{\gamma}(0)=(0,0,0)$ and $(\widetilde{x}, \widetilde{y}, \widetilde{t})=\widetilde{\gamma}(T)$ is the end point of the path $\widetilde{\gamma}$ defined by (5.36) with $\widetilde{\gamma}(0)=(\xi, \eta, \tau)$, then

$$
(\widetilde{x}, \widetilde{y}, \widetilde{t})=(\xi, \eta, \tau) \circ(\bar{x}, \bar{y}, \bar{t})
$$

with $\bar{t}=-T$ (see for instance Corollary 1.2.24 in [7]). The above identity also holds when computing $\widetilde{\gamma}$ at $s=-T$. In particular, if we choose any $\bar{\omega} \in \mathbb{R}^{n}$, and $\bar{t}>0$, we let $T=$ $\bar{t}, \omega(s)=\bar{\omega}$ for any $s \in[-\bar{t}, \bar{t}]$, we find

$$
\begin{aligned}
& \bar{x}=\left(-\bar{t} \bar{\omega},-\frac{\bar{t}^{k+1}}{k+1}|\bar{\omega}|^{k}\right), \quad \tilde{t}=\tau+\bar{t}, \\
& \widetilde{x}=\left(\xi_{1, n}-\bar{t} \bar{\omega}, \xi_{n+1}-\int_{0}^{\bar{t}}\left|\xi_{1, n}-s \bar{\omega}\right|^{k} d s\right) .
\end{aligned}
$$

According with Remark 5.4, the fundamental solution $\widetilde{\Gamma}$ of $\widetilde{\mathscr{L}}$ exists and is invariant with respect to the group operations (5.38) and (5.35). Then function

$$
\Gamma(x, t, \xi, \tau)=\int_{\mathbb{R}^{(k-1) n}} \widetilde{\Gamma}(x, y, t, \xi, 0, \tau) d y
$$

is a fundamental solution to $\mathscr{L}$ and gets from $\widetilde{\Gamma}$ the following invariance properties:

$$
\begin{aligned}
& \Gamma(\widetilde{x}, \widetilde{t}, \xi, \tau)=\Gamma(\bar{x}, \bar{t}, 0,0), \\
& \Gamma\left(\lambda \bar{x}_{1, n}, \lambda^{k+2} \bar{x}_{n+1}, \lambda^{2} \bar{t}, 0_{1, n+1}, 0\right)=\frac{1}{\lambda^{n+k+2}} \Gamma\left(\bar{x}, \bar{t}, 0_{1, n+1}, 0\right),
\end{aligned}
$$

for every $(\xi, \tau)(\bar{x}, \bar{t}) \in \mathbb{R}^{n+2}, \lambda>0$, where $(\widetilde{x}, \widetilde{t})$ is defined in (5.38). In the following remark we summarize the above properties when $(\bar{x}, \bar{t})$ has the form $(5.39)$. 
Remark 5.13 For every $(\xi, \tau) \in \mathbb{R}^{n+2}, t>0$ and for any constant vector $\omega \in \mathbb{R}^{n}$, we have

$$
\Gamma\left(\xi_{1, n}-\sqrt{t} \omega, \xi_{n+1}-\int_{0}^{t}\left|\xi_{1, n}-\frac{s}{\sqrt{t}} \omega\right|^{k} d s, \tau+t, \xi, \tau\right)=\frac{1}{t^{\frac{n+k}{2}+1}} \Gamma\left(-\omega,-\frac{|\omega|^{k}}{k+1}, 0_{1, n+1}, 0\right) .
$$

We next focus on the attainable set $\mathscr{A}_{z_{0}}$ of the unit cylinder

$$
\mathcal{O}=\left\{(x, y, t) \in \mathbb{R}^{k n+2}|| x_{1, n}\left|<1,-1<x_{n+1}<1,\right| y \mid<1,-1<t<1\right\},
$$

with respect to the point $z_{0}=(0,0,0)$. Here $\left|x_{1, n}\right|$ and $|y|$ denote, respectively, the Euclidean norm of the vectors $x_{1, n} \in \mathbb{R}^{n}$ and $y \in \mathbb{R}^{(k-1) n}$.

Unlike the case $k=2$, as $k>2$ we are not able to give a complete characterization of the sets $\mathscr{A}_{z_{0}}$ and $\mathcal{O}_{z_{0}}$ as we did in Lemma 5.11. We will consider instead the differential of the end point map related to (5.36) to find some interior points of $\mathscr{A}_{z_{0}}$. With obvious meaning of the notations, we set $(x(T), y(T), t(T))=\widetilde{\gamma}(T)$, we note that $t(T)=t-T$, and we define

$$
E: L^{2}([0, T]) \rightarrow \mathbb{R}^{k n+1}, \quad E(\omega)=E(\omega, x, y, t, T):=(x(T), y(T)) .
$$

We refer to the classical literature (see e.g. [12, Theorem 3.2.6]) for the differentiability properties of $E$. We next show that the differential $D E(\omega)$ of $E$, computed at some given $\omega \in L^{2}([0, T])$ is surjective. Hence $E(\omega)$ is an interior point of $\mathscr{A}_{z_{0}}$, so that we can apply Theorem 5.2.

Lemma 5.14 Let $\bar{w}$ be any given vector of $\mathbb{R}^{n}$ such that $\bar{w}_{j} \neq 0$ for every $j \in \llbracket 1, n \rrbracket$. Consider the solution $\widetilde{\gamma}$ to the problem (5.36), with $\omega \equiv \bar{w}$. Then $D E(\omega)$ is surjective.

Proof. By the invariance of the vector fields $\widetilde{Y}_{i}, i \in \llbracket 1, n \rrbracket$, and $\widetilde{Z}$ with respect to the homogeneous Lie group $\mathbb{G}$, is not restrictive to assume $(x, y, t)=(0,0,0)$ and $T=1$. To prove our claim, we compute

$$
D E(\omega) \widetilde{\omega}=\lim _{h \rightarrow 0} \frac{1}{h}(E(\omega+h \widetilde{\omega})-E(\omega)),
$$

where

$$
\begin{aligned}
& \widetilde{\omega}(s)=\frac{1}{b-a} v \text { for } s \in[a, b], a, b \in[0,1], a<b, v \text { is any vector of } \mathbb{R}^{n}, \\
& \widetilde{\omega}(s)=0 \text { for } s \notin[a, b] .
\end{aligned}
$$

In the sequel, we denote by $\widetilde{\gamma}^{h}(s)=\left(x^{h}(s), y^{h}(s), t^{h}(s)\right)$ the solution of (5.36) relevant to $\omega+h \widetilde{\omega}$. Clearly, $t^{h}(s)=-s$, and $x^{h}(1)=\bar{w}+h v$, so that

$$
\lim _{h \rightarrow 0} \frac{1}{h}\left(x_{1, n}^{h}(1)-x_{1, n}(1)\right)=v .
$$

We next show that, for every $j \in \llbracket 1, n \rrbracket$ and $i \in \llbracket 1, k-1 \rrbracket$, we have

$$
\lim _{h \rightarrow 0} \frac{y_{i j}^{h}(1)-y_{i j}(1)}{h}=\left(\frac{i}{i+1} \frac{b^{i+1}-a^{i+1}}{b-a}-a \frac{b^{i}-a^{i}}{b-a}+1-b^{i}\right) \bar{w}_{j}^{i-1} v_{j} .
$$


Indeed, we have

$$
\begin{aligned}
y_{i j}^{h}(1) & =\int_{0}^{a}\left(t \bar{w}_{j}\right)^{i} d t+\int_{a}^{b}\left(t \bar{w}_{j}+h \frac{t-a}{b-a} v_{j}\right)^{i} d t+\int_{b}^{1}\left(t \bar{w}_{j}+h v_{j}\right)^{i} d t \\
& =\int_{0}^{a}\left(t \bar{w}_{j}\right)^{i} d t+\int_{a}^{b}\left(t \bar{w}_{j}\right)^{i} d t+\int_{b}^{1}\left(t \bar{w}_{j}\right)^{i} d t \\
& +i h \bar{w}_{j}^{i-1} v_{j}\left(\int_{a}^{b} t^{i-1} \frac{t-a}{b-a} d t+\int_{b}^{1} t^{i-1} d t\right)+o(h), \quad \text { as } h \rightarrow 0, \\
& =y_{i j}(1)+\left(\frac{i}{i+1} \frac{b^{i+1}-a^{i+1}}{b-a}-a \frac{b^{i}-a^{i}}{b-a}+1-b^{i}\right) \bar{w}_{j}^{i-1} v_{j} h+o(h), \quad \text { as } h \rightarrow 0,
\end{aligned}
$$

where $o(h)$ vanishes as $h$ goes to zero. This proves (5.46). Analogously,

$$
\lim _{h \rightarrow 0} \frac{x_{n+1}^{h}(1)-x_{n+1}(1)}{h}=\left(\frac{k}{k+1} \frac{b^{k+1}-a^{k+1}}{b-a}-a \frac{b^{k}-a^{k}}{b-a}+1-b^{k}\right)|\bar{w}|^{k-2}\langle\bar{w}, v\rangle,
$$

when considering system (1.3), and

$$
\lim _{h \rightarrow 0} \frac{x_{n+1}^{h}(1)-x_{n+1}(1)}{h}=\left(\frac{k}{k+1} \frac{b^{k+1}-a^{k+1}}{b-a}-a \frac{b^{k}-a^{k}}{b-a}+1-b^{k}\right) \sum_{j=1}^{n} \bar{w}_{j}^{k-1} v_{j},
$$

in the case of (1.4). Note that for all $i \in \llbracket 1, k \rrbracket$ one has

$$
\frac{i}{i+1} \frac{b^{i+1}-a^{i+1}}{b-a}-a \frac{b^{i}-a^{i}}{b-a}=O(b-a), \quad \text { as } \quad b-a \rightarrow 0,
$$

for any $i \in \llbracket 1, k \rrbracket$. Then, from (5.45), (5.46), (5.47), in the case (1.3), it follows that

$$
\begin{aligned}
D E(\omega) \widetilde{\omega}= & \left(v,\left(1-b^{k}\right)|\bar{w}|^{k-2}\langle\bar{w}, v\rangle,(1-b) v,\left(1-b^{2}\right) \bar{w}_{1} v_{1}, \ldots,\left(1-b^{2}\right) \bar{w}_{n} v_{n},\right. \\
& \left.\ldots,\left(1-b^{k-1}\right) \bar{w}_{1}^{k-2} v_{1}, \ldots,\left(1-b^{k-1}\right) \bar{w}_{n}^{k-2} v_{n}\right)+O(b-a),
\end{aligned}
$$

as $b-a \rightarrow 0$. We next choose $\left.\left.b_{0}, \ldots, b_{k} \in\right] 0,1\right]$ such that $b_{i} \neq b_{m}$ if $i \neq m$ and we let $v$ be any unit vector $e_{j}$ of the canonical basis of $\mathbb{R}^{n}$. Then the $j-t h$, the $n+j+1-t h \ldots$, the $(k-1) n+j+1-t h$ components of $D E(\omega) \widetilde{\omega}$ are

$$
\left(1,1-b_{i},\left(1-b_{i}^{2}\right) w_{j}, \ldots,\left(1-b_{i}^{k-1}\right) \bar{w}_{j}^{k-2}\right),
$$

while the $n+1-t h$ component is $\left(1-b_{i}^{k}\right)|\bar{w}|^{k-2} \bar{w}_{j}$. By our assumption, $\bar{w}_{j} \neq 0$, and the following $(k+1) \times(k+1)$ matrix

$$
M\left(b_{0}, b_{1}, \ldots, b_{k}\right)=\left(\begin{array}{ccccc}
1 & 1-b_{0} & 1-b_{0}^{2} & \ldots & 1-b_{0}^{k} \\
1 & 1-b_{1} & 1-b_{1}^{2} & \ldots & 1-b_{1}^{k} \\
\vdots & \vdots & \vdots & \ddots & \vdots \\
1 & 1-b_{k} & 1-b_{k}^{2} & \ldots & 1-b_{k}^{k}
\end{array}\right) .
$$


is non singular, since

$$
\operatorname{det} M\left(b_{0}, b_{1}, \ldots, b_{k}\right)=(-1)^{k} \prod_{i \neq m}\left(b_{i}-b_{m}\right) \neq 0,
$$

because of our choice of the $b_{i}$ 's. Thus, if we choose $v=e_{j}$ and each $a_{i}$ sufficiently close to $b_{i}$, then (5.50) restores $k+1$ linearly independent vectors. In conclusion, it is possible to find $v_{1}, \ldots, v_{n}, b_{0}, \ldots, b_{k}, a_{0}, \ldots, a_{k}$, such that the vectors $D E(\omega) \widetilde{\omega}$ defined by using $v_{j}, a_{i}, b_{i}$ in (5.44), span $\mathbb{R}^{k n+1}$. This proves our claim for system (1.3). The proof in the case (1.4) is analogous, we only need to replace $(5.47)$ by (5.48). We omit the details.

We next obtain, as a corollary, a Harnack inequality which is invariant with respect to the Lie group $\mathbb{G}=\left(\mathbb{R}^{k n+2}, \circ,\left(\widetilde{\delta}_{\lambda}\right)_{\lambda>0}\right)$. For every compact subset $\mathcal{K}$ of the unit cylinder $\mathcal{O}$ defined in (5.42), any positive $r$ and any $z_{0}=\left(x_{0}, y_{0}, t_{0}\right) \in \mathbb{R}^{k n+2}$ we set

$$
\mathcal{O}_{r}\left(z_{0}\right)=z_{0} \circ \widetilde{\delta}_{r} \mathcal{O}=\left\{z_{0} \circ \widetilde{\delta}_{r} \zeta \mid \zeta \in \mathcal{O}\right\}, \quad \mathcal{K}_{r}\left(z_{0}\right)=z_{0} \circ \widetilde{\delta}_{r} \mathcal{K} .
$$

We recall the definition of the end-point map $E$ introduced in (5.43), and we define the following sets

$$
\begin{aligned}
\widetilde{I}:= & \left\{\omega:\left[0, \frac{1}{2}\right] \rightarrow \mathbb{R}^{n} \mid \omega(s) \equiv \widetilde{\omega} \text { with } \frac{1}{4} \leq\left|\widetilde{\omega}_{j}\right| \leq 1, j \in \llbracket 1, n \rrbracket\right\}, \\
\widehat{I}:= & \left\{\omega:\left[0, \frac{1}{2}\right] \rightarrow \mathbb{R}^{n} \mid \omega_{j}(s)=\hat{c} \text { for any } s \in\left[0, \frac{1}{8}\right] \cup\left[\frac{3}{8}, \frac{1}{2}\right],\right. \\
& \left.\omega_{j}(s)=-\hat{c} \text { for any } s \in\left[\frac{1}{8}, \frac{3}{8}\right], j \in \llbracket 1, n \rrbracket \text { with } 1 \leq \hat{c} \leq \sqrt{2},\right\}, \\
\widetilde{\mathcal{K}}:= & \left\{\left(E\left(\omega, 0,0,0, \frac{1}{2}\right), \frac{1}{2}\right) \mid \omega \in \widetilde{I}\right\} \quad \widehat{\mathcal{K}}:=\left\{\left(E\left(\omega, 0,0,0, \frac{1}{2}\right), \frac{1}{2}\right) \mid \omega \in \widehat{I}\right\} .
\end{aligned}
$$

We remark that $\widetilde{\mathcal{K}}$ and $\widehat{\mathcal{K}}$ are compact subset of the unit cylinder defined in (5.42), being $E$ a continuous map. We also note that, if we denote by $\bar{z}=(\bar{x}, \bar{y}, \bar{t})$ the point $\left(E\left(\omega, 0,0,0, \frac{1}{2}\right), \frac{1}{2}\right)$ with $\omega$ as described in $\widehat{I}$, we have $\bar{x}_{1, n}=0_{1, n}, \bar{x}_{n+1}=\hat{c}^{k} a_{n+1, k}$, with:

$$
\begin{array}{ll}
a_{n+1, k}=\frac{4 n^{k / 2}}{k+1} 8^{-(k+1)} & \text { for (5.8), } \\
a_{n+1, k}=\frac{4 n}{k+1} 8^{-(k+1)} & \text { for (5.9) and } k \text { even, } \\
a_{n+1, k}=0 & \text { for (5.9) and } k \text { odd. }
\end{array}
$$

Proposition 5.15 Let $\mathcal{O} \subseteq \mathbb{R}^{k n+2}$ be the unit cylinder defined in (5.42), let $r>0$ and let $z_{0}=\left(x_{0}, y_{0}, t_{0}\right) \in \mathbb{R}^{N+1}$. If $\widetilde{\mathscr{L}}$ is the lifted operator of $\mathscr{L}$ in $(5.8)$ or $(5.9)$, then there exists a positive constant $\widetilde{c}$ such that the sets $\widetilde{\mathcal{K}}$ and $\widehat{\mathcal{K}}$ defined in $(5.52)$ are compact subsets of $\operatorname{Int}\left(\mathscr{A}_{(0,0,0)}\right)$. Moreover there exist two positive constants $C_{\widetilde{\mathcal{K}}}, C_{\widehat{\mathcal{K}}}$, only depending on $\mathcal{O}$ and on $\widetilde{\mathscr{L}}$, such that

$$
\sup _{\widetilde{\mathcal{K}}_{r}\left(z_{0}\right)} \widetilde{u} \leq C_{\widetilde{\mathcal{K}}} \widetilde{u}\left(z_{0}\right), \quad \sup _{\widehat{\mathcal{K}}_{r}\left(z_{0}\right)} \widetilde{u} \leq C_{\widehat{\mathcal{K}}} \widetilde{u}\left(z_{0}\right),
$$

for every positive solution $\widetilde{u}$ of $\widetilde{L} \widetilde{u}=0$ in $\mathcal{O}_{r}\left(z_{0}\right)$.

Proof. By the invariance of $\widetilde{\mathscr{L}}$ with respect to the homogeneous Lie group $\mathbb{G}$, it is not restrictive to assume $\left(x_{0}, y_{0}, t_{0}\right)=(0,0,0)$ and $r=1$. By Lemma $5.14 E(\omega)$ is an interior point of $\mathscr{A}_{(0,0,0)}$ for any $\omega \in \widetilde{I}$, and for any $\omega \in \widehat{I}$. The conclusion follows from Theorem 5.2. 


\section{Harnack chains and lower bounds}

In this section we build Harnack chains and we prove asymptotic lower bounds for positive solutions to $\mathscr{L} u=0$. In the first Lemma 6.1 we capture paths that give Gaussian lower bounds as $\left|x_{1, n}-\xi_{1, n}\right|^{2} \geq K(t-\tau)$, for suitably big $K$ and asymptotic bounds for points $x, \xi$ with $\left|x_{n+1}-\xi_{n+1}\right|$ suitably big with respect to $(t-\tau)^{1+k / 2}$.

Lemma 6.2 applies when $\left|x_{n+1}-\xi_{n+1}\right|$ is small with respect to $(t-\tau)^{1+k / 2}$. Moreover, in this lemma, we consider points with non degenerate components set to zero. In such case, if we denote $(\bar{x}, \bar{y}, \bar{t})=(\widetilde{x}, \widetilde{y}, \widetilde{t}) \circ(\widehat{x}, \widehat{y}, \widehat{t})$, then

$$
\widetilde{x}_{1, n}=\widehat{x}_{1, n}=0_{1, n} \quad \Longrightarrow \quad \bar{x}_{n+1}=\widetilde{x}_{n+1}+\widehat{x}_{n+1},
$$

that is the group law $\circ$ is additive w.r.t. the $(n+1)$ th component. In some sense this allows to move in the direction of the vector field $\underbrace{\left[\partial_{x_{1}},\left[\partial_{x_{1}}, \cdots,\left[\partial_{x_{1}}, Z\right] \cdots\right]\right]}_{k \text { times }}=k ! \partial_{x_{n+1}}$.

The proof of the two lemmas is based on the lifting procedure introduced in Section 5 and on the construction of a finite sequence of cylinders contained in the lifted domain of the solution. Specifically, we find points along the trajectory of the integral path introduced in (5.36). The bounds depend on the length of the Harnack chain that in turns depends on the slope of the trajectory. Asymptotic lower bounds are proved in Propositions 6.3 and 6.4.

Lemma 6.1 Let $\mathscr{L}$ be the operator defined in (5.8) or in (5.9), let $T_{1}, \tau, t, T_{2}$ be such that $T_{1}<\tau<t<T_{2}$ and $t-\tau \leq \tau-T_{1}$. Let $\left.\gamma:[0, t-\tau] \rightarrow \mathbb{R}^{n+1} \times\right] T_{1}, T_{2}$ [ be a path satisfying

$$
\gamma^{\prime}(s)=\sum_{j=1}^{n} \omega_{j} Y_{j}(\gamma(s))+Z(\gamma(s)), \quad \gamma(0)=(x, t), \quad \gamma(t-\tau)=(\xi, \tau)
$$

for some constant vector of $\mathbb{R}^{n}$ such that

$$
\max _{j \in \llbracket 1, n \rrbracket}\left|\omega_{j}\right| \leq 2 \min _{i \in \llbracket 1, n \rrbracket}\left|\omega_{i}\right|, \quad \text { and that } \quad(t-\tau) \max _{j \in \llbracket 1, n \rrbracket} \omega_{j}^{2} \geq 4 .
$$

Then there exists a positive constant $C$, only depending on $\mathscr{L}$, such that:

$$
u(\xi, \tau) \leq \exp \left(C\left((t-\tau) \max _{j \in \llbracket 1, n \rrbracket} \omega_{j}^{2}+1\right)\right) u(x, t),
$$

for every non-negative solution u to $\mathscr{L} u=0$ in $\left.\mathbb{R}^{n+1} \times\right] T_{1}, T_{2}[$.

Proof. Define the function $\widetilde{u}$ by setting $\widetilde{u}(x, y, t)=u(x, t)$ for every $\left.(x, y, t) \in \mathbb{R}^{k n+1} \times\right] T_{1}, T_{2}[$. Clearly, $\widetilde{u}$ is a non-negative solution to $\widetilde{\mathscr{L}} \widetilde{u}=0$. Let $\left.\widetilde{\gamma}:[0, t-\tau] \rightarrow \mathbb{R}^{k n+1} \times\right] T_{1}, T_{2}[$ be the solution of the Cauchy problem

$$
\left\{\begin{array}{l}
\widetilde{\gamma}^{\prime}(s)=\sum_{j=1}^{n} \omega_{j} \widetilde{Y}_{j}(\widetilde{\gamma}(s))+\widetilde{Z}(\widetilde{\gamma}(s)), \quad s \in[0, t-\tau] \\
\widetilde{\gamma}(0)=(x, 0, t)
\end{array}\right.
$$


where $\omega$ is the constant vector in (6.2). Note that, if $\widetilde{x}_{1, n+1}$ and $x_{1, n+1}$ are the first $n+1$ components of $\widetilde{\gamma}$ and $\gamma$, respectively, then we have $\widetilde{x}_{1, n+1}(s)=x_{1, n+1}(s)$, for every $s \in[0, t-\tau]$.

We next apply the Harnack inequalities stated in Proposition 5.15 to a suitable set of points $z_{1}, \ldots, z_{m}$ lying on $\widetilde{\gamma}([0, t-\tau])$. We suppose $\omega_{1}^{2}=\max _{j \in \llbracket 1, n \rrbracket} \omega_{j}^{2}$, as it is not restrictive, and we let $m$ be the unique positive integer such that $m-1<\frac{(t-\tau) \omega_{1}^{2}}{2} \leq m$. We set $\widetilde{s}=\frac{t-\tau}{m}$ and we define $z_{j}=\widetilde{\gamma}(j \widetilde{s})$ for $j \in \llbracket 1, m \rrbracket$. In order to apply Proposition 5.15, we put $\widetilde{r}=\sqrt{2 \widetilde{s}}$, and $\widetilde{\omega}=\frac{\widetilde{r}}{2} \omega$. Note that $m \geq \frac{(t-\tau) \omega_{1}^{2}}{2} \geq 2$ and, as a consequence, we have $\widetilde{\omega} \in \widetilde{I}$. Thus, if we denote by $\widetilde{z}$ the point $\left(E\left(\widetilde{\omega}, 0,0,0, \frac{1}{2}\right), \frac{1}{2}\right)$ defined in (5.52), we have $\widetilde{z} \in \widetilde{\mathcal{K}}$. Moreover, $z_{j}=z_{j-1} \circ \delta_{\widetilde{r}} \widetilde{z}$, thus

$$
z_{j} \in \widetilde{\mathcal{K}}_{\widetilde{r}}\left(z_{j-1}\right), \quad \text { for any } j \in \llbracket 1, m \rrbracket .
$$

Note that by our assumption, $\frac{\widetilde{r}^{2}}{2} \leq \frac{2}{\omega_{1}^{2}} \leq t-\tau \leq \tau-T_{1}$, hence, $\left.\mathcal{O}_{\widetilde{r}}\left(z_{j}\right) \subset \mathbb{R}^{k n+1} \times\right] T_{1}, T_{2}[$ for every $j \in \llbracket 0, m-1 \rrbracket$. Thus, by Proposition 5.15, there exists a constant $C_{\widetilde{\mathcal{K}}}>1$ such that $\widetilde{u}\left(z_{j}\right) \leq C_{\widetilde{\mathcal{K}}} \widetilde{u}\left(z_{j-1}\right)$ for every $j \in \llbracket 1, m \rrbracket$. In particular, being $m<\frac{(t-\tau) \omega_{1}^{2}}{2}+1, z_{0}=$ $(x, 0, t), z_{m}=\widetilde{\gamma}(t-\tau)$, we find

$$
\widetilde{u}(\widetilde{\gamma}(t-\tau)) \leq C_{\widetilde{\mathcal{K}}}^{\frac{(t-\tau) \omega_{1}^{2}}{2}+1} \widetilde{u}(x, 0, t) .
$$

Hence,

$$
u(\xi, \tau)=u(\gamma(t-\tau))=\widetilde{u}(\widetilde{\gamma}(t-\tau)) \leq C_{\widetilde{\mathcal{K}}}^{\frac{(t-\tau) \omega_{1}^{2}}{2}+1} \widetilde{u}(x, 0, t)=C_{\widetilde{\mathcal{K}}}^{\frac{(t-\tau) \omega_{1}^{2}}{2}+1} u(x, t),
$$

and our claim follows by choosing $C:=\log \left(C_{\widetilde{\mathcal{K}}}\right)$.

Note that, whenever Lemma 6.1 applies, we have $x_{1, n}(t-\tau)=x_{1, n}+(t-\tau) \omega \neq x_{1, n}$. Next result gives a bound along a trajectory $\widetilde{\gamma}$ such that $x_{1, n}(s)=0$ for any $s \in[0, t-\tau]$.

Lemma 6.2 Let $\mathscr{L}$ be the operator defined in (5.8) or in (5.9), with $k$ even, and let $a_{n+1, k}$ be as in (5.53). Let $T_{1}, \tau, t, T_{2}$ be such that $T_{1}<\tau<t<T_{2}$ and $t-\tau \leq \tau-T_{1}$. Then there exists a positive constant $C$, only depending on $\mathscr{L}$, such that:

$$
u\left(0_{1, n}, x_{n+1}+\widetilde{\xi}_{n+1}, \tau\right) \leq \exp \left(C\left(\frac{(t-\tau)^{1+2 / k}}{\widetilde{\xi}_{n+1}^{2 / k}}+1\right)\right) u\left(0_{1, n}, x_{n+1}, t\right) .
$$

for every $\left.(x, t) \in \mathbb{R}^{n+1} \times\right] T_{1}, T_{2}[, \tau \in] T_{1}, t\left[\right.$ with $\left.\widetilde{\xi}_{n+1} \in\right] 0,2 a_{n+1, k}(t-\tau)^{1+k / 2}[$, for every non-negative solution $u$ to $\mathscr{L} u=0$ in $\left.\mathbb{R}^{n+1} \times\right] T_{1}, T_{2}[$.

Proof. As in the proof of Lemma 6.1, we consider the function $\widetilde{u}$ defined as $\widetilde{u}(x, y, t)=u(x, t)$ for every $\left.(x, y, t) \in \mathbb{R}^{k n+1} \times\right] T_{1}, T_{2}[$. Let $m$ be the unique positive integer such that

$$
m-1<(2(t-\tau))^{1+2 / k}\left(\frac{a_{n+1, k}}{\widetilde{\xi}_{n+1}}\right)^{2 / k} \leq m .
$$


Let $\widetilde{r}=\sqrt{\frac{2(t-\tau)}{m}}$ and let $\hat{c}=\left(\frac{\widetilde{\xi}_{n+1}}{a_{n+1, k}}\right)^{1 / k} \frac{m^{1 / 2}}{(2(t-\tau))^{1 / 2+1 / k}}$. Note that from our assumption $0<\widetilde{\xi}_{n+1}<2 a_{n+1, k}(t-\tau)^{1+k / 2}$ it follows that $m \geq 2$, hence $1 \leq \hat{c} \leq \sqrt{2}$. Then, if we denote by $\bar{z}$ the point $\left(E\left(\omega, 0,0,0, \frac{1}{2}\right), \frac{1}{2}\right)$ defined in (5.52), with $\hat{c}$ as above, we have $\bar{z} \in \widehat{\mathcal{K}}$.

Let $z_{0}=\left(0_{1, n}, x_{n+1}, 0, t\right)$, and let $z_{j}=z_{j-1} \circ \delta_{\widetilde{r}} \bar{z}$, for $j \in \llbracket 1, m \rrbracket$. By our assumption we also have $\widetilde{r}^{2} \leq \tau-T_{1}$, then $\left.\mathcal{O}_{\widetilde{r}}\left(z_{j}\right) \subset \mathbb{R}^{k n+1} \times\right] T_{1}, T_{2}$, for every $j \in \llbracket 0, m \rrbracket$. Thus Proposition 5.15 yields $\widetilde{u}\left(z_{m}\right) \leq C_{\widetilde{\mathcal{K}}}^{m} \widetilde{u}\left(z_{0}\right)$. According with (6.1), and with our choice of $m, \hat{c}$, and $\widetilde{r}$, the first $n+1$ components of $z_{m}$ are $\left(0_{1, n}, x_{n+1}+\widetilde{\xi}_{n+1}\right)$. Then

$$
u\left(0_{1, n}, x_{n+1}+\widetilde{x}_{n+1}, \tau\right)=\widetilde{u}\left(z_{m}\right) \leq C_{\widetilde{\mathcal{K}}}^{m} \widetilde{u}\left(z_{0}\right)=C_{\widetilde{\mathcal{K}}}^{m} u\left(0_{1, n}, x_{n+1}, t\right),
$$

and the conclusion follows from (6.3).

Proposition 6.3 Let $\mathscr{L}$ be the operator defined in (5.8) and let $k$ be a positive even integer. Let $\left.u: \mathbb{R}^{n+1} \times\right] T_{1}, T_{2}[\rightarrow \mathbb{R}$ be a non-negative solution to $\mathscr{L} u=0$, and let $t, \tau \in \mathbb{R}$ be such that $T_{1}<\tau<t<T_{2}$, and $t-\tau \leq 2\left(\tau-T_{1}\right)$. Then there exists a positive constant $C_{1}$, only depending on $\mathscr{L}$, such that

i) for any $x, \xi \in \mathbb{R}^{n+1}$ such that $\xi_{n+1}-x_{n+1} \geq 15^{k}\left((t-\tau)\left(\left|x_{1, n}\right|^{k}+\left|\xi_{1, n}\right|^{k}\right)+n^{k / 2}(t-\tau)^{1+k / 2}\right)$ we have

$$
u(\xi, \tau) \leq \exp \left(C_{1}\left(\frac{\left|x_{1, n}-\xi_{1, n}\right|^{2}}{t-\tau}+\frac{\left(\xi_{n+1}-x_{n+1}-\frac{t-\tau}{2^{k+4}}\left(\left|x_{1, n}\right|^{k}+\left|\xi_{1, n}\right|^{k}\right)\right)^{2 / k}}{(t-\tau)^{1+2 / k}}+1\right)\right) u(x, t)
$$

ii) for any $x, \xi \in \mathbb{R}^{n+1}$ such that $0<\xi_{n+1}-x_{n+1} \leq \frac{t-\tau}{2(k+1)}\left(\left|x_{1, n}\right|^{k}+\left|\xi_{1, n}\right|^{k}\right)+\frac{n^{k / 2}}{8^{k+1}(k+1)}(t-$ $\tau)^{1+k / 2}$ we have

$$
u(\xi, \tau) \leq \exp \left(C_{1}\left(\frac{\left|x_{1, n}\right|^{k+2}+\left|\xi_{1, n}\right|^{k+2}}{\xi_{n+1}-x_{n+1}}+\frac{(t-\tau)^{1+2 / k}}{\left(\xi_{n+1}-x_{n+1}\right)^{2 / k}}+1\right)\right) u(x, t) .
$$

Proof of (i). We divide the proof into two steps. In the first one we find a path $\gamma:\left[0, \frac{t-\tau}{2}\right] \rightarrow$ $\mathbb{R}^{k n+2}$ that steers $x_{1, n}$ to $\xi_{1, n}$. In the second step another path $\gamma_{1}+\gamma_{2}$ steers the $(n+1) t h$ component $x_{n+1}\left(\frac{t-\tau}{2}\right)$ of $\gamma\left(\frac{t-\tau}{2}\right)$ to $\xi_{n+1}$.

Step 1: If $\omega=\frac{2}{t-\tau}\left(x_{1, n}-\xi_{1, n}\right)$ satisfies the assumptions of Lemma 6.1, then one readily gets $x_{1, n}\left(\frac{t-\tau}{2}\right)=\xi_{1, n}$ and $u\left(\gamma\left(\frac{t-\tau}{2}\right)\right) \leq \exp \left(C\left[2 \frac{\left|x_{1, n}-\xi_{1, n}\right|^{2}}{t-\tau}+1\right]\right) u(x, t)$ and the first step is achieved. If this is not the case, we rely on the following construction. Set $K=$ $\max _{j \in \llbracket 1, n \rrbracket} \frac{\left|\xi_{j}-x_{j}\right|}{\sqrt{t-\tau}}$ and $M:=\max \left\{3 K, \frac{3}{2}\right\}$. For every $j \in \llbracket 1, n \rrbracket$, we set

$$
\widetilde{\omega}_{j}:=\frac{4 M}{\sqrt{t-\tau}}, \quad \widehat{\omega}_{j}:=4 \frac{\xi_{j}-x_{j}}{t-\tau}-\frac{4 M}{\sqrt{t-\tau}},
$$


so that

$$
\frac{8}{3} \frac{M}{\sqrt{t-\tau}} \leq\left|\widehat{\omega}_{j}\right| \leq \frac{16}{3} \frac{M}{\sqrt{t-\tau}}, \quad \frac{t-\tau}{4} \widetilde{\omega}_{j}^{2} \geq 9, \quad \frac{t-\tau}{4} \widehat{\omega}_{j}^{2} \geq 4
$$

Consider now the path $\gamma$ associated to (6.2) with $\omega(s)=\widetilde{\omega} \mathbb{I}_{s \in\left[0, \frac{t-\tau}{4}\right]}+\widehat{\omega} \mathbb{I}_{s \in\left[\frac{t-\tau}{4}, \frac{t-\tau}{2}\right]}$ for which $x_{1, n}\left(\frac{t-\tau}{2}\right)=\xi_{1, n}$. The assumptions of Lemma 6.1 are clearly satisfied. Hence:

$$
\begin{aligned}
u\left(\gamma\left(\frac{t-\tau}{2}\right)\right) & \leq \exp \left(C^{\prime}\left(\frac{\left|x_{1, n}-\xi_{1, n}\right|^{2}}{t-\tau}+1\right)\right) u(x, t), \\
\gamma\left(\frac{t-\tau}{2}\right) & =\left(\xi_{1, n}, x_{n+1}+\int_{0}^{\frac{t-\tau}{2}}\left|x_{1, n}+\int_{0}^{s} \omega(u) d u\right|^{k} d s, \frac{t+\tau}{2}\right),
\end{aligned}
$$

for some positive constant $C^{\prime}$. By a plain change of variable in the above integral we find

$$
x_{n+1}\left(\frac{t-\tau}{2}\right)-x_{n+1}=\int_{0}^{\frac{t-\tau}{4}}\left|x_{1, n}+s \widetilde{\omega}\right|^{k} d s+\int_{0}^{\frac{t-\tau}{4}}\left|\xi_{1, n}-s \widehat{\omega}\right|^{k} d s .
$$

Note that, for $s \in\left[0, \frac{t-\tau}{4}\right]$ and $j \in \llbracket 1, n \rrbracket$, we have

$$
\begin{aligned}
& \left|x_{j}+s \widetilde{\omega}_{j}\right| \leq\left|x_{j}\right|+\frac{4 s}{\sqrt{t-\tau}} M \leq\left|x_{j}\right|+3\left(\left|x_{j}-\xi_{j}\right|+\frac{\sqrt{t-\tau}}{2}\right) \leq 4\left|x_{j}\right|+3\left|\xi_{j}\right|+3 \frac{\sqrt{t-\tau}}{2} \\
& \left|\xi_{j}-s \widehat{\omega}_{j}\right| \leq\left|\xi_{j}\right|+\frac{4 s}{\sqrt{t-\tau}}\left(M+\frac{\left|x_{j}-\xi_{j}\right|}{\sqrt{t-\tau}}\right) \leq 4\left|x_{j}\right|+5\left|\xi_{j}\right|+3 \frac{\sqrt{t-\tau}}{2}
\end{aligned}
$$

thus, from the elementary inequality $\left(a^{2}+b^{2}+c^{2}\right)^{k / 2} \leq 3^{k / 2-1}\left(a^{k}+b^{k}+c^{k}\right)$, we get

$$
x_{n+1}\left(\frac{t-\tau}{2}\right)-x_{n+1} \leq \frac{t-\tau}{4} 3^{k-1}\left(2^{2 k+1}\left|x_{1, n}\right|^{k}+\left(3^{k}+5^{k}\right)\left|\xi_{1, n}\right|^{k}+2\left(\frac{3^{k} n^{k / 2}}{2^{k}}\right)(t-\tau)^{k / 2}\right) .
$$

Recalling that $\xi_{n+1}-x_{n+1} \geq 15^{k}\left((t-\tau)\left(\left|x_{1, n}\right|^{k}+\left|\xi_{1, n}\right|^{k}\right)+n^{k / 2}(t-\tau)^{1+k / 2}\right)$, we find

$$
\xi_{n+1}-x_{n+1}\left(\frac{t-\tau}{2}\right) \geq \frac{t-\tau}{2} 15^{k}\left(\left|x_{1, n}\right|^{k}+\left|\xi_{1, n}\right|^{k}+n^{k / 2}(t-\tau)^{k / 2}\right) .
$$

We next prove a similar lower bound for $x_{n+1}\left(\frac{t-\tau}{2}\right)-x_{n+1}$. To this aim, we note that, if $\left|x_{1, n}\right| \geq \frac{t-\tau}{8}|\widetilde{\omega}|$, then $\left|x_{1, n}+s \widetilde{\omega}\right| \geq \frac{1}{2}\left|x_{1, n}\right|$ for every $s \in\left[0, \frac{t-\tau}{16}\right]$. Analogously, if $\left|x_{1, n}\right| \leq$ $\frac{t-\tau}{8}|\widetilde{\omega}|$, then $\left|x_{1, n}+s \widetilde{\omega}\right| \geq \frac{t-\tau}{16}|\widetilde{\omega}|$ for every $s \in\left[\frac{3}{16}(t-\tau), \frac{t-\tau}{4}\right]$. The same remark holds if we replace $x_{1, n}$ and $\widetilde{\omega}$ by $\xi_{1, n}$ and $\widehat{\omega}$, respectively. As a consequence we find,

$$
x_{n+1}-x_{n+1}\left(\frac{t-\tau}{2}\right) \geq \frac{t-\tau}{16}\left(\max \left\{\frac{\left|x_{1, n}\right|^{k}}{2^{k}},\left(\frac{t-\tau}{16}|\widetilde{\omega}|\right)^{k}\right\}+\max \left\{\frac{\left|\xi_{1, n}\right|^{k}}{2^{k}},\left(\frac{t-\tau}{16}|\widehat{\omega}|\right)^{k}\right\}\right),
$$

so that, in particular,

$$
x_{n+1}\left(\frac{t-\tau}{2}\right)-x_{n+1} \geq \frac{t-\tau}{2^{k+4}}\left(\left|x_{1, n}\right|^{k}+\left|\xi_{1, n}\right|^{k}\right) .
$$


Step 2: We next denote by $\bar{\omega}$ the vector in $\mathbb{R}^{n}$ such that $\bar{\omega}_{j}=1$ if $\xi_{j} \geq 0, \bar{\omega}_{j}=-1$ if $\xi_{j}<0$, for $j \in \llbracket 1, n \rrbracket$, and we fix a real parameter $b \geq \frac{4}{\sqrt{t-\tau}}$, that will be specified later. We consider the path $\left.\gamma_{1}:\left[\frac{t-\tau}{2}, \frac{3}{4}(t-\tau)\right] \rightarrow \mathbb{R}^{n+1} \times\right] T_{1}, T_{2}\left[\right.$, starting from $\gamma\left(\frac{t-\tau}{2}\right)$ and defined as in (6.2) with $\omega=b \bar{\omega}$, then the path $\left.\gamma_{2}:\left[\frac{3}{4}(t-\tau), t-\tau\right] \rightarrow \mathbb{R}^{n+1} \times\right] T_{1}, T_{2}[$, starting from $\gamma_{1}\left(\frac{3}{4}(t-\tau)\right)$ and defined by setting $\omega=-b \bar{\omega}$. From Lemma 6.1 it then follows

$$
u\left(\xi_{1, n}, \varphi(b), \tau\right) \leq \exp \left(2 C\left(\frac{(t-\tau) b^{2}}{4}+1\right)\right) u\left(\gamma\left(\frac{t-\tau}{2}\right)\right),
$$

where

$$
\varphi(b)=x_{n+1}\left(\frac{t-\tau}{2}\right)+2 \int_{0}^{\frac{t-\tau}{4}}\left|\xi_{1, n}+s b \bar{\omega}\right|^{k} d s
$$

is an increasing continuous function of $b \in\left[\frac{4}{\sqrt{t-\tau}},+\infty[\right.$. An elementary computation shows that

$$
2 \int_{0}^{\frac{t-\tau}{4}}\left|\xi_{1, n}+s b \bar{\omega}\right|^{k} d s \leq 2^{k} \frac{t-\tau}{4}\left|\xi_{1, n}\right|^{k}+\frac{b^{k} n^{k / 2}}{k+1} \frac{(t-\tau)^{k+1}}{2^{k+2}}
$$

then

$$
\varphi\left(\frac{4}{\sqrt{t-\tau}}\right) \leq x_{n+1}\left(\frac{t-\tau}{2}\right)+2^{k} \frac{t-\tau}{4}\left|\xi_{1, n}\right|^{k}+\frac{2^{k-2} n^{k / 2}}{k+1}(t-\tau)^{1+k / 2}<\xi_{n+1},
$$

by (6.7). On the other hand we have

$$
\varphi(b) \geq x_{n+1}\left(\frac{t-\tau}{2}\right)+\frac{b^{k}}{k+1} \frac{(t-\tau)^{k+1} n^{k / 2}}{2^{2 k+1}} \rightarrow+\infty, \quad \text { as } b \rightarrow+\infty .
$$

Hence, there exists a unique $\widetilde{b} \geq \frac{4}{\sqrt{t-\tau}}$ such that $\varphi(\widetilde{b})=\xi_{n+1}$. Moreover from (6.10) it also follows that

$$
\widetilde{b} \leq 2(k+1)^{1 / k}\left(\frac{2}{t-\tau}\right)^{1+1 / k}\left(\xi_{n+1}-x_{n+1}\left(\frac{t-\tau}{2}\right)\right)^{1 / k} n^{-1 / 2},
$$

which, together with (6.8), gives

$$
\widetilde{b} \leq(k+1)^{1 / k}\left(\frac{2}{t-\tau}\right)^{1+1 / k}\left(\xi_{n+1}-x_{n+1}-\frac{t-\tau}{2^{k+4}}\left(\left|x_{1, n}\right|^{k}+\left|\xi_{1, n}\right|^{k}\right)\right)^{1 / k} .
$$

Eventually, equation (6.9) yields

$$
u(\xi, \tau) \leq \exp \left(C_{1}\left(\frac{\left(\xi_{n+1}-x_{n+1}-\frac{t-\tau}{2^{k+4}}\left(\left|x_{1, n}\right|^{k}+\left|\xi_{1, n}\right|^{k}\right)\right)^{2 / k}}{(t-\tau)^{1+2 / k}}+1\right)\right) u\left(\gamma\left(\frac{t-\tau}{2}\right)\right) .
$$

with $C_{1}=4^{1+1 / k}(k+1)^{2 / k} C$. The above inequality, with (6.4), proves the claim (i).

Proof of (ii). We prove our claim by applying Lemma 6.2 in a suitable interval $\left[\tau+t_{2}, t-t_{1}\right] \subsetneq$ $[\tau, t]$, and Lemma 6.1 in the remaining intervals $\left[t-t_{1}, t\right]$ and $\left[\tau, \tau+t_{2}\right]$. We first suppose 
that $x_{1, n} \neq 0, \xi_{1, n} \neq 0$, we set

$$
\begin{aligned}
& t_{1}=\min \left\{\frac{\max _{j \in \llbracket 1, n \rrbracket} x_{j}^{2}}{4}, \frac{\xi_{n+1}-x_{n+1}}{\left|x_{1, n}\right|^{k}}, \frac{t-\tau}{3}\right\}, \\
& t_{2}=\min \left\{\frac{\max _{j \in \llbracket 1, n \rrbracket} \xi_{j}^{2}}{4}, \frac{\xi_{n+1}-x_{n+1}}{\left|\xi_{1, n}\right|^{k}}, \frac{t-\tau}{3}\right\},
\end{aligned}
$$

and we consider the paths

$$
\begin{aligned}
& \gamma_{1}(s)=\left(\left(1-\frac{s}{t_{1}}\right) x_{1, n}, x_{n+1}+\frac{\left(s-t_{1}\right)^{k+1}+t_{1}^{k+1}}{(k+1) t_{1}^{k}}\left|x_{1, n}\right|^{k}, t-s\right), \quad s \in\left[0, t_{1}\right] \\
& \gamma_{2}(s)=\left(\frac{s}{t_{2}} \xi_{1, n}, \xi_{n+1}+\frac{s^{k+1}-t_{2}^{k+1}}{(k+1) t_{2}^{k}}\left|\xi_{1, n}\right|^{k}, \tau+t_{2}-s\right), \quad s \in\left[0, t_{2}\right] .
\end{aligned}
$$

We next proceed assuming that $\max _{j \in \llbracket 1, n \rrbracket}\left|x_{j}\right| \leq 2 \min _{i \in \llbracket 1, n \rrbracket}\left|x_{i}\right|$ and $\max _{j \in \llbracket 1, n \rrbracket}\left|\xi_{j}\right| \leq$ $2 \min _{i \in \llbracket 1, n \rrbracket}\left|\xi_{i}\right|$. In this case we apply Lemma 6.1 in the interval $\left[0, t_{1}\right]$ with $\bar{\omega}=-\frac{1}{t_{1}} x_{1, n}$, so that we have $t_{1} \max _{j \in \llbracket 1, n \rrbracket} \bar{\omega}_{j}^{2} \geq 4$ and $\max _{j \in \llbracket 1, n \rrbracket}\left|\bar{\omega}_{j}\right| \leq 2 \min _{i \in \llbracket 1, n \rrbracket}\left|\bar{\omega}_{i}\right|$. We find

$$
\begin{aligned}
u\left(\gamma_{1}\left(t_{1}\right)\right) & \leq \exp \left(C\left(\max \left\{\frac{\left|x_{1, n}\right|^{k+2}}{\xi_{n+1}-x_{n+1}}, 3 \frac{\left|x_{1, n}\right|^{2}}{t-\tau}, 4\right\}+1\right)\right) u(x, t), \\
u(\xi, \tau) & \leq \exp \left(C\left(\max \left\{\frac{\left|\xi_{1, n}\right|^{k+2}}{\xi_{n+1}-x_{n+1}}, 3 \frac{\left|\xi_{1, n}\right|^{2}}{t-\tau}, 4\right\}+1\right)\right) u\left(\gamma_{2}(0)\right),
\end{aligned}
$$

with

$$
\gamma_{1}\left(t_{1}\right)=\left(0, x_{n+1}+\frac{t_{1}}{k+1}\left|x_{1, n}\right|^{k}, t-t_{1}\right), \quad \gamma_{2}(0)=\left(0, \xi_{n+1}-\frac{t_{2}}{k+1}\left|\xi_{1, n}\right|^{k}, \tau+t_{2}\right) .
$$

If $\max _{j \in \llbracket 1, n \rrbracket}\left|x_{j}\right|>2 \min _{i \in \llbracket 1, n \rrbracket}\left|x_{i}\right|$, we rely on the argument used at the beginning of the proof of (i). Specifically, we set $M:=\max \left\{3 \max _{j \in \llbracket 1, n \rrbracket} \frac{\left|x_{j}\right|}{\sqrt{t_{1}}}, \frac{3}{2}\right\}$, and $\widetilde{\omega}_{j}:=2 \frac{M}{\sqrt{t_{1}}}, \widehat{\omega}_{j}:=\frac{2}{t_{1}} x_{j}+2 \frac{M}{\sqrt{t_{1}}}$ for every $j \in \llbracket 1, n \rrbracket$, then we consider the path $\gamma$ associated to $(6.2)$ with $\omega(s)=\widetilde{\omega} \mathbb{I}_{s \in\left[0, \frac{t_{1}}{2}\right]}-$ $\widehat{\omega} \mathbb{I}_{s \in\left[\frac{t_{1}}{2}, t_{1}\right]}$. Also in this case we get (6.11), with some bigger constant $C$. Aiming to simplify our exposition we omit the details of the proof.

We next conclude the proof by using Lemma 6.2. We set $\widetilde{\xi}_{n+1}=\xi_{n+1}-x_{n+1}-\frac{t_{1}}{k+1}\left|x_{1, n}\right|^{k}-$ $\frac{t_{2}}{k+1}\left|\xi_{1, n}\right|^{k}$, and we recall that $\xi_{n+1}-x_{n+1} \leq \frac{t-\tau}{2(k+1)}\left(\left|x_{1, n}\right|^{k}+\left|\xi_{1, n}\right|^{k}\right)+\frac{n^{k / 2}}{8^{k+1}(k+1)}(t-\tau)^{1+k / 2}$. Thus

$$
\frac{t-\tau}{3}<\left(t-t_{1}\right)-\left(\tau+t_{2}\right)<t-\tau, \quad \frac{k-1}{k+1}\left(\xi_{n+1}-x_{n+1}\right) \leq \widetilde{\xi}_{n+1} \leq \frac{n^{k / 2}}{8^{k+1}(k+1)}(t-\tau)^{1+k / 2}
$$

Then, from Lemma 6.2 we get

$$
\begin{aligned}
u\left(\gamma_{2}(0)\right) & \leq \exp \left(C\left(\frac{\left(t-t_{1}-t_{2}-\tau\right)^{1+2 / k}}{\left(\widetilde{\xi}_{n+1}\right)^{2 / k}}+1\right)\right) u\left(\gamma\left(t_{1}\right)\right) \\
& \leq \exp \left(C\left(\left(\frac{k+1}{k-1}\right)^{2 / k} \frac{(t-\tau)^{1+2 / k}}{\left(\xi_{n+1}-x_{n+1}\right)^{2 / k}}+1\right)\right) u\left(\gamma\left(t_{1}\right)\right) .
\end{aligned}
$$


From inequalities (6.11) and (6.13) it follows that

$$
u(\xi, \tau) \leq \exp \left(C^{\prime}\left(\frac{\left|x_{1, n}\right|^{k+2}+\left|\xi_{1, n}\right|^{k+2}}{\xi_{n+1}-x_{n+1}}+\frac{(t-\tau)^{1+2 / k}}{\left(\xi_{n+1}-x_{n+1}\right)^{2 / k}}+\frac{\left|x_{1, n}\right|^{2}+\left|\xi_{1, n}\right|^{2}}{t-\tau}+1\right)\right) u(x, t),
$$

for some positive constant $C^{\prime}$ only depending on $C$ and on $k$. Note that the last term in the above expression is bounded by the first one. Indeed, the inequality

$$
\frac{\left|x_{1, n}\right|^{2}+\left|\xi_{1, n}\right|^{2}}{t-\tau} \leq \frac{2}{k+2} \frac{\left|x_{1, n}\right|^{k+2}+\left|\xi_{1, n}\right|^{k+2}}{(t-\tau)^{1+k / 2}}+\frac{k}{k+2}
$$

combined with (6.12), gives

$$
\frac{\left|x_{1, n}\right|^{2}+\left|\xi_{1, n}\right|^{2}}{t-\tau} \leq \frac{2}{4^{k+1}(k+2)(k-1)} \frac{\left|x_{1, n}\right|^{k+2}+\left|\xi_{1, n}\right|^{k+2}}{\xi_{n+1}-x_{n+1}}+\frac{k}{k+2} .
$$

This concludes the proof of (ii) when $x_{1, n} \neq 0$, and $\xi_{1, n} \neq 0$.

If $x_{1, n}=0$, we simply omit the construction of $\gamma_{1}$, and we rely on $\gamma_{2}$ and on the application of Lemma 6.2 in the interval $\left[\tau+t_{2}, t\right]$. Analogously, if $\xi_{1, n}=0$, we avoid the construction of $\gamma_{2}$. This concludes the proof.

Proposition 6.4 Let $\mathscr{L}$ be the operator defined in (5.9) and let $k$ be a positive integer. Let $\left.u: \mathbb{R}^{n+1} \times\right] T_{1}, T_{2}[\rightarrow \mathbb{R}$ be a non-negative solution to $\mathscr{L} u=0$, and let $t, \tau \in \mathbb{R}$ be such that $T_{1}<\tau<t<T_{2}$, and $t-\tau \leq 2\left(\tau-T_{1}\right)$. Then there exists a positive constant $C_{1}$, only depending on $\mathscr{L}$, such that

i) if $k$ is even, then for any $x, \xi \in \mathbb{R}^{n+1}$ such that $\xi_{n+1}-x_{n+1} \geq 15^{k}(t-\tau) \sum_{j=1}^{n}\left(x_{j}^{k}+\xi_{j}^{k}\right)+$ $n\left(\frac{3}{2}\right)^{k}(t-\tau)^{1+k / 2}$ we have

$$
\begin{aligned}
u(\xi, \tau) \leq \exp ( & C_{1}\left(\frac{\left|x_{1, n}-\xi_{1, n}\right|^{2}}{t-\tau}+\right. \\
& \left.\left.+\frac{\left(\xi_{n+1}-x_{n+1}-\frac{1}{2^{k+4}} \sum_{j=1}^{n}\left(x_{j}^{k}+\xi_{j}^{k}\right)(t-\tau)\right)^{2 / k}}{(t-\tau)^{1+2 / k}}+1\right)\right) u(x, t) ;
\end{aligned}
$$

ii) if $k$ is even, then for any $x, \xi \in \mathbb{R}^{n+1}$ such that $0<\xi_{n+1}-x_{n+1} \leq \frac{t-\tau}{2(k+1)} \sum_{j=1}^{n}\left(x_{j}^{k}+\xi_{j}^{k}\right)+$ $\frac{(t-\tau)^{1+k / 2}}{4^{k+1}(k+1)}$ we have

$$
u(\xi, \tau) \leq \exp \left(C_{1}\left(\frac{\left|x_{1, n}\right|^{k+2}+\left|\xi_{1, n}\right|^{k+2}}{\xi_{n+1}-x_{n+1}}+\frac{(t-\tau)^{1+2 / k}}{\left(\xi_{n+1}-x_{n+1}\right)^{2 / k}}+1\right)\right) u(x, t) ;
$$

iii) if $k$ is odd, we have

$$
\begin{aligned}
u(\xi, \tau) \leq \exp ( & C_{1}\left(\frac{\left|x_{1, n}-\xi_{1, n}\right|^{2}}{t-\tau}+\right. \\
& \left.\left.+\frac{\left|\xi_{n+1}-x_{n+1}-\frac{1}{2^{k+4}} \sum_{j=1}^{n}\left(x_{j}^{k}+\xi_{j}^{k}\right)(t-\tau)\right|^{2 / k}}{(t-\tau)^{1+2 / k}}+1\right)\right) u(x, t) .
\end{aligned}
$$


Proof. The proof of (i) and (ii) is analogous to that of Proposition 6.3. The unique modification is due to the fact that here we have

$$
x_{n+1}\left(\frac{t-\tau}{2}\right)-x_{n+1}=\int_{0}^{\frac{t-\tau}{4}} \sum_{j=1}^{n}\left(x_{j}+s \widetilde{\omega}_{j}\right)^{k} d s+\int_{0}^{\frac{t-\tau}{4}} \sum_{j=1}^{n}\left(\xi_{j}-s \widehat{\omega}_{j}\right)^{k} d s .
$$

instead of (6.5). From (6.6), by the same argument used in the proof of (6.7) and (6.8), we obtain

$$
\frac{t-\tau}{2^{k+4}} \sum_{j=1}^{n}\left(x_{j}^{k}+\xi_{j}^{k}\right) \leq x_{n+1}\left(\frac{t-\tau}{2}\right)-x_{n+1} \leq \frac{t-\tau}{2} 15^{k} \sum_{j=1}^{n}\left(\left|x_{j}\right|^{k}+\left|\xi_{j}\right|^{k}\right)+2 n\left(\frac{3}{2}\right)^{k}(t-\tau)^{k / 2+1} .
$$

We omit the other details of the proof of (i) and (ii).

The proof of (iii) follows from the same argument used in the proof of (i). Note that, as $k$ is odd, the function

$$
\varphi(b)=x_{n+1}\left(\frac{t-\tau}{2}\right)+2 \int_{0}^{\frac{t-\tau}{4}} \sum_{j=1}^{n}\left(x_{j}+b \bar{\omega}_{j}\right)^{k} d s
$$

defined for any $b \in \mathbb{R}$ is surjective, then in this case Lemma 6.1 is sufficient to conclude the proof.

\section{Final derivation of the estimates}

Proof of Theorem 2.1. It follows from the bounds proved in Sections 4.5 and 6. Consider first equation (1.3).

The upper bound of (i) is given in (4.38). In order to prove the lower bound, we fix a constant vector $\left.\omega \in \mathbb{R}^{n}, \alpha \in\right] 0,1[$, and we set

$$
\widetilde{x}_{1, n}=\xi_{1, n}-\alpha \sqrt{t} \omega, \quad \widetilde{x}_{n+1}=\xi_{n+1}-\int_{0}^{\alpha^{2} t}\left|\xi_{1, n}-\frac{s}{\alpha \sqrt{t}} \omega\right|^{k} d s .
$$

According with Remark 5.13, we have

$$
p\left(\alpha^{2} t, \widetilde{x}, \xi\right)=\Gamma\left(\widetilde{x}, \alpha^{2} t, \xi, 0\right)=\frac{C}{\left(\alpha^{2} t\right)^{1+\frac{n+k}{2}}}, \quad C=\Gamma\left(\omega,-\frac{|\omega|^{k}}{k+1}, 1\right)
$$

For our purpose, we choose here $\omega=(1, \ldots, 1) \in \mathbb{R}^{n}$, and $\alpha=1 / \sqrt{2}$. Note that the constant $C$ is strictly positive, being $\omega \neq 0$. Also note that

$$
0<\xi_{n+1}-\widetilde{x}_{n+1} \leq 2^{k} \alpha^{2}\left(t\left|\xi_{1, n}\right|^{k}+\alpha^{k} t^{1+k / 2}|\omega|^{k}\right) .
$$

We then apply Proposition 6.3 (i), and we find

$$
\begin{aligned}
p(t, x, \xi) \geq \exp \left(-C_{1}\right. & \left(\frac{\left|x_{1, n}-\widetilde{x}_{1, n}\right|^{2}}{t}+\right. \\
& \left.\left.\frac{\left(\widetilde{x}_{n+1}-x_{n+1}-\frac{t}{2^{k+5}}\left(\left|x_{1, n}\right|^{k}+\left|\widetilde{x}_{1, n}\right|^{k}\right)\right)^{2 / k}}{t^{1+2 / k}}+1\right)\right) p(t / 2, \widetilde{x}, \xi) .
\end{aligned}
$$


The lower bound (i) thus follows from the inequalities $\left|\widetilde{x}_{1, n}-\xi_{1, n}\right| \leq \sqrt{t} / 2|\omega|$ and $\xi_{n+1}>\widetilde{x}_{n+1}$.

The upper bound of (ii) is equation (4.37), the lower bound is given in Lemma 4.11.

The upper bound of (iii) is given in (4.39). In order to prove the lower bound we rely on Proposition 6.3 (ii). In order to satisfy its hypothesis, we choose $\alpha \in] 0,1 / \sqrt{2}]$ such that $\xi_{n+1}-\widetilde{x}_{n+1} \leq \frac{1}{2}\left(\xi_{n+1}-x_{n+1}\right)$. Note that

$$
0<\xi_{n+1}-\widetilde{x}_{n+1} \leq 2^{k} \alpha^{2}\left(t\left|\widetilde{x}_{1, n}\right|^{k}+\alpha^{k} t^{1+k / 2}|\omega|^{k}\right)
$$

then it is sufficient to choose $\alpha$ such that

$$
\alpha^{2} t \leq \frac{\xi_{n+1}-x_{n+1}}{2^{k+2}\left|\widetilde{x}_{1, n}\right|^{k}}, \quad(\alpha \sqrt{t})^{k+2} \leq \frac{\xi_{n+1}-x_{n+1}}{2^{k+2}|\omega|^{k}} .
$$

Using our assumption $\left|\xi_{n+1}-x_{n+1}\right| \leq K t^{1+k / 2}$ we find

$$
\frac{1}{\alpha^{2} t} \geq \frac{C_{K, k}}{t} \max \left(\frac{\left|\widetilde{x}_{1, n}\right|^{k}}{\left|\xi_{n+1}-x_{n+1}\right|^{\frac{k}{k+2}}}, 1\right)
$$

for some positive constant $C_{K, k}$ depending on $K$ and $k$. Then (6.16) gives

$$
p\left(\alpha^{2} t, \widetilde{x}, \xi\right) \geq \frac{\widetilde{C}_{K, k}}{t^{\frac{n+k+2}{2}}}\left(1+\frac{\left|\widetilde{x}_{1, n}\right|^{k+2}}{\left|\xi_{n+1}-x_{n+1}\right|}\right)^{\frac{(k+2)(n+k+2)}{2 k}}
$$

With this choice of $\alpha$ Proposition 6.3 (ii) then gives

$$
\begin{aligned}
p(t, x, \xi) \geq & \frac{\widetilde{C}_{K, k}}{t^{\frac{n+k+2}{2}}}\left(1+\frac{\left|\widetilde{x}_{1, n}\right|^{k+2}}{\left|\xi_{n+1}-x_{n+1}\right|}\right)^{\frac{(k+2)(n+k+2)}{2 k}} \cdot \\
& \exp \left(-C_{1}\left(\frac{\left|x_{1, n}\right|^{k+2}+\left|\widetilde{x}_{1, n}\right|^{k+2}}{\widetilde{x}_{n+1}-x_{n+1}}+\frac{\left(\left(1-\alpha^{2}\right) t\right)^{1+2 / k}}{\left(\widetilde{x}_{n+1}-x_{n+1}\right)^{2 / k}}+1\right)\right)
\end{aligned}
$$

and our lower bound follows from the inequalities $t / 2 \leq\left(1-\alpha^{2}\right) t \leq t, 1 / 2\left(\xi_{n+1}-x_{n+1}\right) \leq$ $\widetilde{x}_{n+1}-x_{n+1} \leq \xi_{n+1}-x_{n+1}$ and $\left|\widetilde{x}_{1, n}-\xi_{1, n}\right| \leq \sqrt{t} / 2|\omega|$.

When considering equation (1.4), the lower bounds for points (i) and (iii) directly follow from 6.4. The proof of the remaining bounds can be done by the same arguments used for equation (1.3).

Remark 6.5 We can assume by symmetry that w.l.o.g. $\left|\xi_{1, n}\right| \geq\left|x_{1, n}\right|$. In this case, observe from equation (6.17) that if $\left|\xi_{1, n}\right| \geq K t^{1 / 2}$ for $K$ large enough, then we can derive from Lemma 4.11 that the Gaussian diagonal regime holds for the lower bound. From the proof leading to (4.38), this means that the non-exponential estimates in case i) could be alternatively rewritten changing the $t^{-\left\{\frac{n+k}{2}+1\right\}}$ term into

$$
t^{-n / 2} t^{-3 / 2}\left(\left\{\left|x_{1, n}\right|^{k-1}+\left|\xi_{1, n}\right|^{k-1}\right\} \vee t^{\frac{k-1}{2}}\right)^{-1},
$$

that emphasizes the regime transition depending on the magnitude of the non-degenerate components w.r.t. to their characteristic time-scale. From the above computations, the expression in (6.19) can also substitute the non-exponential term in case iii). 
Acknowledgments. We express our gratitude to Denis Talay, because this study has started in occasion of a workshop he organized, with the specific aim at fostering new research. We thank E. Lanconelli for his interest in our work. Also, the last author thanks University Paris 7 and the Laboratoire de Probabilités et Modèles Aléatoires for the one month invitation during which this work developed.

\section{References}

[1] V. BALLY, On the connection between the Malliavin covariance matrix and Hörmander's condition, J. Funct. Anal., 96-2 (1990), pp. 219-255.

[2] V. Bally and A. Kohatsu-Higa, Lower bounds for densities of Asian type stochastic differential equations, J. Funct. Anal., 258 (2010), pp. 3134-3164.

[3] V. Bally And D. TAlay, The law of the Euler scheme for stochastic differential equations: I. Convergence rate of the distribution function, Prob. Th. Rel. Fields, 104-1 (1996), pp. 43-60.

[4] H. Bauer, Harmonische Räume und ihre Potentialtheorie, Ausarbeitung einer im Sommersemester 1965 an der Universität Hamburg gehaltenen Vorlesung. Lecture Notes in Mathematics, No. 22, Springer-Verlag, Berlin, 1966.

[5] G. Ben Arous and R. LÉAndre, Décroissance exponentielle du noyau de la chaleur sur la diagonale. II, Probab. Theory Related Fields, 90 (1991), pp. 377-402.

[6] A. Bonfiglioli and E. Lanconelli, Lie groups related to Hörmander operators and Kolmogorov-Fokker-Planck equations, Commun. Pure Appl. Anal., 11 (2012), pp. 15871614.

[7] A. Bonfiglioli, E. Lanconelli, and F. Uguzzoni, Stratified Lie groups and potential theory for their sub-Laplacians, Springer Monographs in Mathematics, Springer, Berlin, 2007.

[8] A. Bonfiglioli and F. Uguzzoni, Maximum principle and propagation for intrinsicly regular solutions of differential inequalities structured on vector fields, J. Math. Anal. Appl., 322 (2006), pp. 886-900.

[9] J. M. Bony, Principe du maximum, inégalité de Harnack et unicité du problème de Cauchy pour les opérateurs elliptiques dégénérés, Ann. Inst. Fourier, 19 (1969), pp. $277-$ 304 .

[10] A. N. Borodin And P. SAlminen, Handbook of Brownian motion-facts and formulae, Probability and its Applications, Birkhäuser Verlag, Basel, second ed., 2002.

[11] U. Boscain And S. Polidoro, Gaussian estimates for hypoelliptic operators via optimal control, Atti Accad. Naz. Lincei Cl. Sci. Fis. Mat. Natur. Rend. Lincei (9) Mat. Appl., 18 (2007), pp. 333-342. 
[12] A. Bressan And B. Piccoli, Introduction to the mathematical theory of control, vol. 2 of AIMS Series on Applied Mathematics, American Institute of Mathematical Sciences (AIMS), Springfield, MO, 2007.

[13] A. Carciola, A. Pascucci, and S. Polidoro, Harnack inequality and no-arbitrage bounds for self-financing portfolios, Bol. Soc. Esp. Mat. Apl. SëMA, (2009), pp. 19-31.

[14] C. Cinti and E. Lanconelli, Riesz and Poisson-Jensen representation formulas for a class of ultraparabolic operators on Lie groups, Potential Anal., 30 (2009), pp. 179-200.

[15] C. Cinti, K. Nyström, And S. Polidoro, A note on Harnack inequalities and propagation sets for a class of hypoelliptic operators, Potential Anal., 33 (2010), pp. 341-354.

[16] C. Cinti And S. Polidoro, Harnack inequalities and lifting procedure for evolution hypoelliptic equations, Lecture Notes of Seminario Interdisciplinare di Matematica, 7 (2008), pp. 93-105.

[17] _ Pointwise local estimates and Gaussian upper bounds for a class of uniformly subelliptic ultraparabolic operators, J. Math. Anal. Appl., 338 (2008), pp. 946-969.

[18] C. Constantinescu And A. Cornea, Potential theory on harmonic spaces, SpringerVerlag, New York, 1972. With a preface by H. Bauer, Die Grundlehren der mathematischen Wissenschaften, Band 158.

[19] F. Delarue and S. Menozzi, Density estimates for a random noise propagating through a chain of differential equations, J. Funct. Anal., 259 (2010), pp. 1577-1630.

[20] F. Flandoli, Random perturbation of PDEs and fluid dynamic models, vol. 2015 of Lecture Notes in Mathematics, Springer, Heidelberg, 2011. Lectures from the 40th Probability Summer School held in Saint-Flour, 2010.

[21] N. Ikeda And S. Watanabe, Stochastic differential equations, North Holland, 1989.

[22] S. Janson, Gaussian Hilbert spaces, vol. 129 of Cambridge Tracts in Mathematics, Cambridge University Press, Cambridge, 1997.

[23] M. KaC, On distributions of certain Wiener functionals, Trans. Amer. Math. Soc., 65 (1949), pp. 1-13.

[24] A. E. Kogoj And E. Lanconelli, An invariant Harnack inequality for a class of hypoelliptic ultraparabolic equations, Mediterr. J. Math., 1 (2004), pp. 51-80.

[25] A. Kolmogoroff, Zufällige Bewegungen (zur Theorie der Brownschen Bewegung), Ann. of Math. (2), 35 (1934), pp. 116-117.

[26] S. Kusuoka and D. Stroock, Applications of the Malliavin calculus. I, Stochastic analysis (Katata/Kyoto, 1982), North-Holland Math. Library, 32 (1984), pp. 271-306.

[27] _ Applications of the Malliavin calculus. II, J. Fac. Sci. Univ. Tokyo Sect. IA Math, 32 (1985), pp. 1-76. 
[28] — Applications of the Malliavin calculus. III, J. Fac. Sci. Univ. Tokyo Sect. IA Math., 34 (1987), pp. 391-442.

[29] P. Malliavin, $C^{k}$-hypoellipticity with degeneracy, In: Stochastic analysis (Proc. Internat. Conf., Northwestern Univ., Evanston, Ill., 1978), Academic Press, New YorkLondon, (1978), pp. 199-214.

[30] _ Stochastic calculus of variation and hypoelliptic operators, In: Proceedings of the International Symposium on Stochastic Differential Equations (Res. Inst. Math. Sci., Kyoto Univ., Kyoto, 1976), Wiley, New York-Chichester-Brisbane, (1978), pp. 195-263.

[31] J. R. Norris, Simplified Malliavin Calculus, Séminaire de Probabilités, XX (1986), pp. 101-130.

[32] D. Nualart, The Malliavin calculus and related topics. Probability and its Applications, Springer-Verlag, New York, 1995.

[33] D. Nualart, Analysis on Wiener space and anticipating stochastic calculus, in Lectures on probability theory and statistics (Saint-Flour, 1995), LNM 1690. Springer, Berlin, 1998, pp. 123-227.

[34] D. Revuz And M. Yor, Continuous martingales and Brownian motion. 3rd ed., Grundlehren der Mathematischen Wissenschaften. 293. Berlin: Springer, 1999.

[35] L. P. Rothschild And E. M. Stein, Hypoelliptic differential operators and nilpotent groups, Acta Math., 137 (1976), pp. 247-320.

[36] N. Smirnov, Sur la distribution de $\omega^{2}$ (criterium de M. von Mises), C. R. Acad. Sci. Paris, 202 (1936), pp. 449-452.

[37] D. Stroock, Some applications of stochastic calculus to partial differential equations, In: Eleventh Saint Flour probability summer school-1981 (Saint Flour, 1981), Lecture Notes in Math., 976, Springer, Berlin, (1983), pp. 267-382.

[38] D. W. Stroock, Homogeneous chaos revisited, 1247 (1987), pp. 1-7.

[39] L. Tolmatz, Asymptotics of the distribution of the integral of the absolute value of the Brownian bridge for large arguments, Ann. Probab., 28 (2000), pp. 132-139.

[40] E. TRÉLAT, Some properties of the value function and its level sets for affine control systems with quadratic cost, J. Dynam. Control Systems, 6 (2000), pp. 511-541. 